\title{
INTEGRATED OPTIC PLATFORM FOR PHOTONIC CRYSTAL DEVICES
}

\author{
by
}

Kristian E. Medri

B. A. Sc. (University of Toronto, 2003)

M. A. Sc. (Ryerson University, 2006)

A thesis submitted to the Faculty of Graduate and Postdoctoral Affairs in partial fulfillment of the requirements for the degree of

Doctor of Philosophy

in

Electrical and Computer Engineering

Ottawa-Carleton Institute for Electrical Engineering

Department of Electronics

Carleton University

Ottawa, Canada

(C) 2012, Kristian E. Medri 
Library and Archives

Canada

Published Heritage

Branch

395 Wellington Street

Ottawa ON K1A ON4

Canada
Bibliothèque et

Archives Canada

Direction du

Patrimoine de l'édition

395 , rue Wellington

Ottawa ON K1A ON4

Canada
Your file Votre référence

ISBN: 978-0-494-94232-1

Our file Notre référence

ISBN: 978-0-494-94232-1
NOTICE:

The author has granted a nonexclusive license allowing Library and Archives Canada to reproduce, publish, archive, preserve, conserve, communicate to the public by telecommunication or on the Internet, loan, distrbute and sell theses worldwide, for commercial or noncommercial purposes, in microform, paper, electronic and/or any other formats.

The author retains copyright ownership and moral rights in this thesis. Neither the thesis nor substantial extracts from it may be printed or otherwise reproduced without the author's permission.
AVIS:

L'auteur a accordé une licence non exclusive permettant à la Bibliothèque et Archives Canada de reproduire, publier, archiver, sauvegarder, conserver, transmettre au public par télécommunication ou par l'Internet, prêter, distribuer et vendre des thèses partout dans le monde, à des fins commerciales ou autres, sur support microforme, papier, électronique et/ou autres formats.

L'auteur conserve la propriété du droit d'auteur et des droits moraux qui protege cette thèse. $\mathrm{Ni}$ la thèse ni des extraits substantiels de celle-ci ne doivent être imprimés ou autrement reproduits sans son autorisation.
In compliance with the Canadian Privacy Act some supporting forms may have been removed from this thesis.

While these forms may be included in the document page count, their removal does not represent any loss of content from the thesis.
Conformément à la loi canadienne sur la protection de la vie privée, quelques formulaires secondaires ont été enlevés de cette thèse.

Bien que ces formulaires aient inclus dans la pagination, il n'y aura aucun contenu manquant. 


\section{Abstract}

This research explores loading optical dielectric waveguides with patterned thin photonic crystal overlays of higher dielectric value than the waveguide. The asymmetry of the overlaid waveguide region is designed such that the original waveguide retains the field maximum within its boundaries but produces a sizeable field contribution in the photonic crystal such that the properties of photonic crystals can be exploited. The enabling feature is that the peak transition and the effective index transition are discovered to be not coincident for increasing propagation constant. The waveguide dimensions are chosen such that when configured as a channel they are compatible for efficient coupling to optical fibres. Materials are chosen with fabrication techniques in mind. The theoretical analysis focuses on optimizing the coupling of the waveguide to the overlay. Three techniques of modeling the behavior of a high index contrast 1D photonic crystal overlay on glass are examined. The findings for $1 \mathrm{D}$ can be expanded for more complex photonic crystal designs to be built on the same platform. Finite Difference Time Domain (FDTD) simulation is found to be the most appropriate for the tuning of high dielectric contrast overlay designs. The sensitivity of light to variations in the refractive index, thickness, period, and length of patterned overlay are examined using FDTD simulations. Transmission and reflection spectrums are obtained using active and passive optical device configurations which can be optimized based on the Bragg response shape and filter edge location. Novel devices created by a structured overlay such as distributed Bragg filters are modeled. A glass-based slab waveguide, coated with a thin patterned high dielectric overlay, is configured into a refractive index sensor. The asymmetric nature of the waveguide configuration is exploited by keeping the mode in the slab ii 
waveguide while enhancing the field level in the overlay-superstrate. An index of refraction sensitivity of up to one part in $10^{5}$ is determined using the FDTD simulation technique. The nature of the sensor ensures optical fibre compatibility, requires sub- $\mu \mathrm{L}$ sample volumes and provides a high resolution. This theoretical analysis demonstrates value in future fabrication of the described novel devices. 


\section{Acknowledgements}

I would like to thank Professor Robert C. Gauthier for serving as my supervisor at Carleton University. I also thank my examination committee.

I am grateful to my colleagues for the opportunity to discuss engineering theory and to the others in the laboratory for intellectual discussion from time to time. Many thanks to my family and friends for their support and help without which completing this degree would not have been possible. 


\section{Table of Contents}

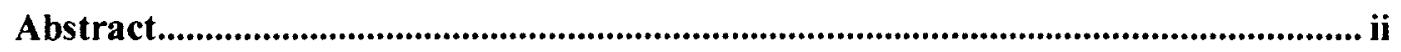

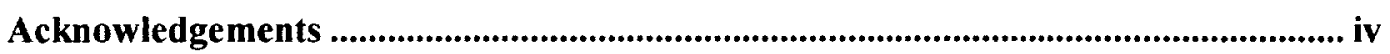

Table of Contents ..........................................................................................................

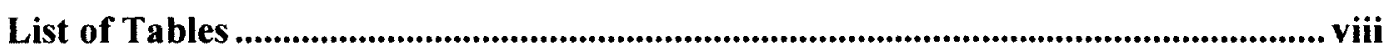

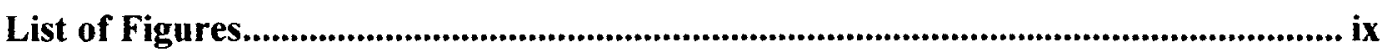

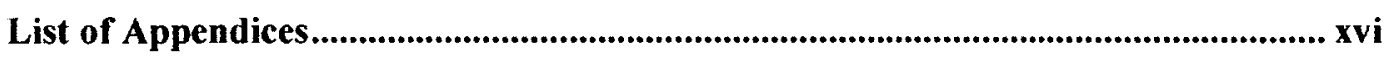

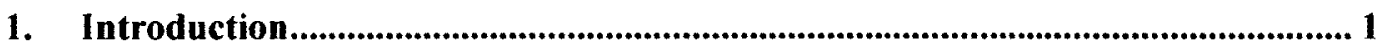

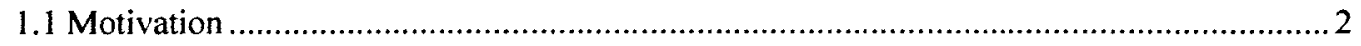

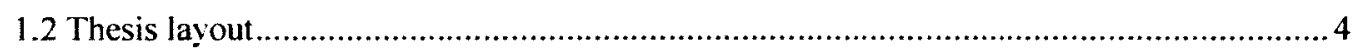

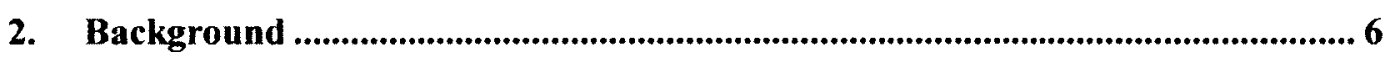

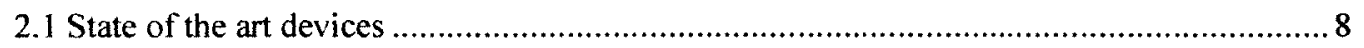

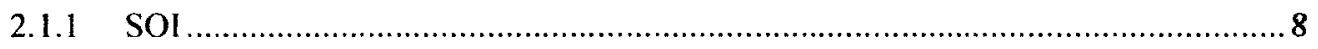

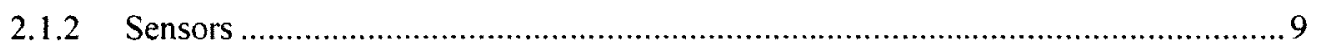

2.1.3 Benefits of high refractive index dielectric cladding ....................................... 10

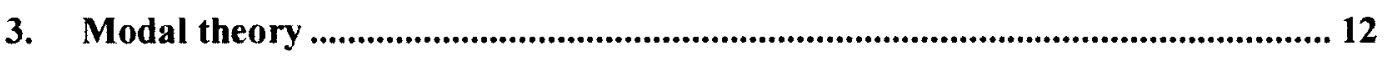

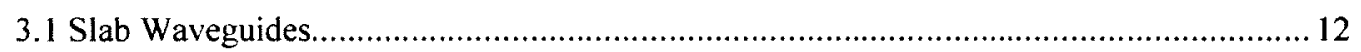

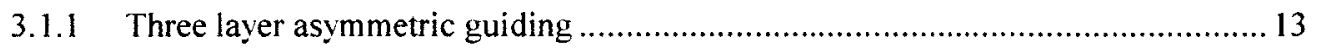

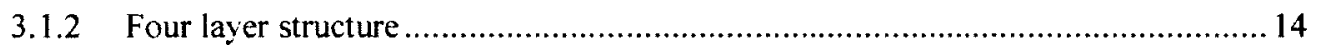

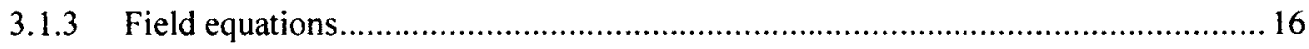

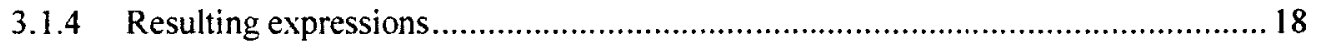

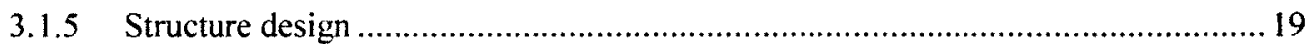

3.2 Multimode device considerations for thin high index dielectric overlay slab waveguides. 23

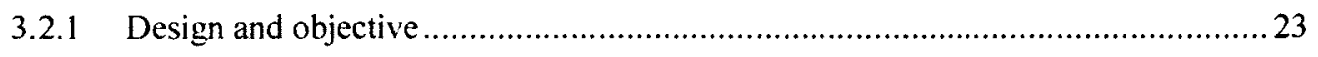




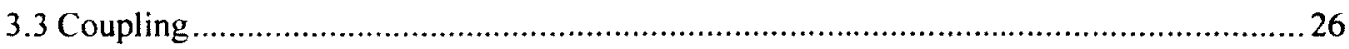

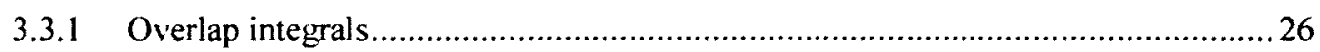

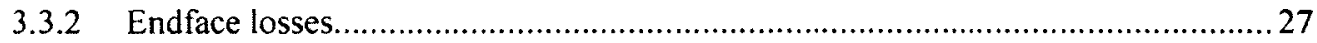

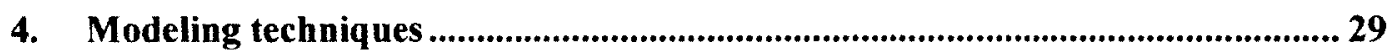

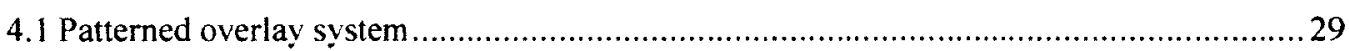

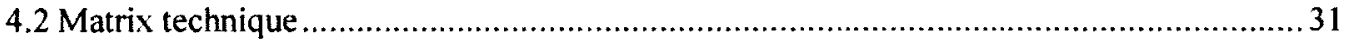

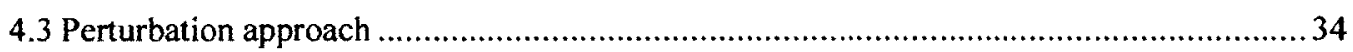

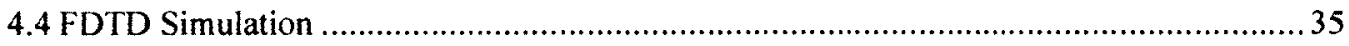

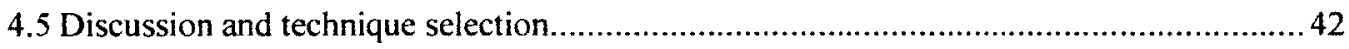

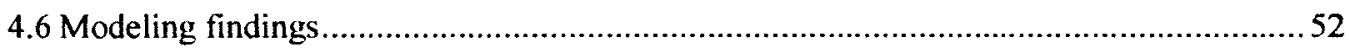

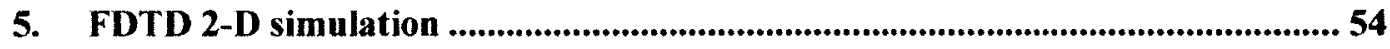

5.1 Application of FDTD to the patterned overlay system …................................................54

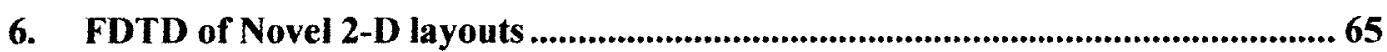

6.1 Patterned overlays: thin silicon layer applied to glass waveguides ..................................65

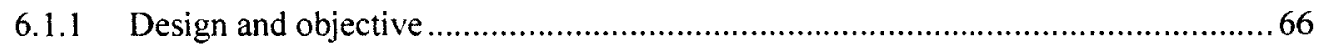

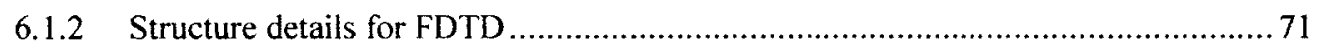

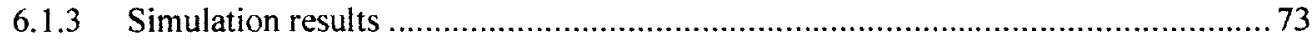

6.1.4 Comparison and performance evaluation ..................................................... 78

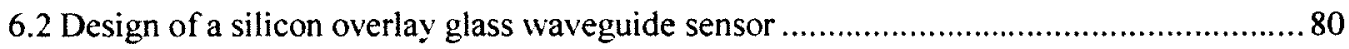

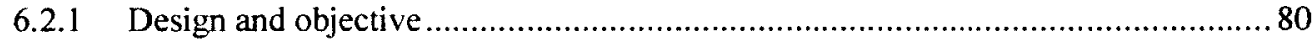

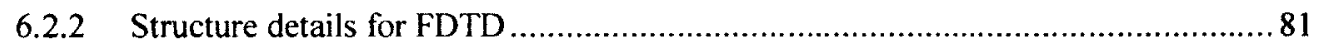

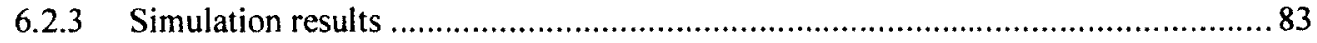

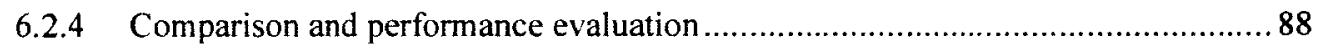

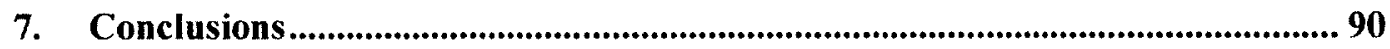

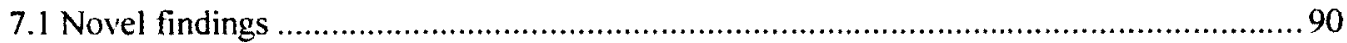

vi 


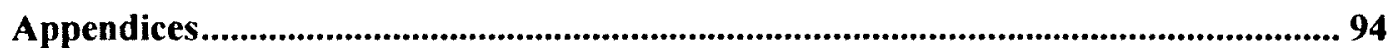

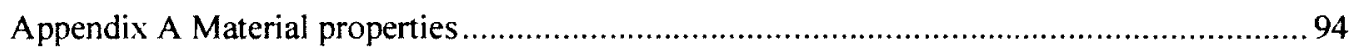

Appendix B Discussion regarding extending analysis to 3-D...............................................96

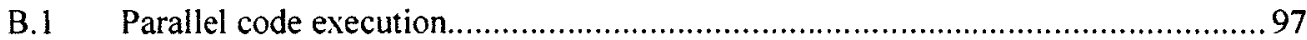

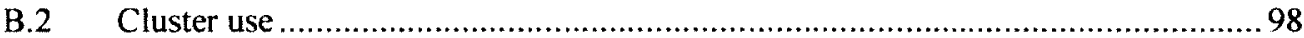

B.3 Expected response for channel structure ....................................................... 101

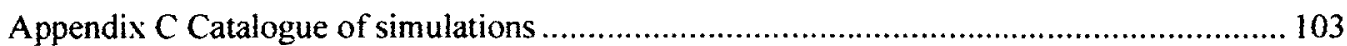

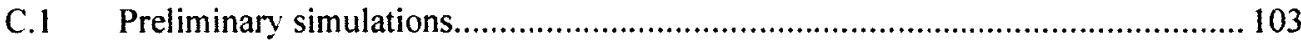

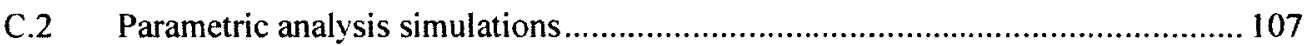

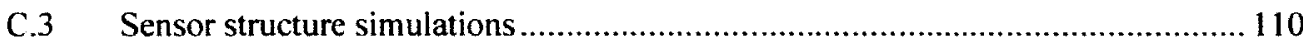

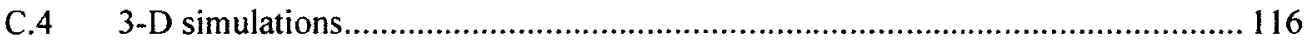

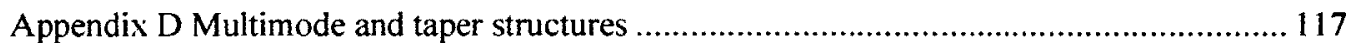

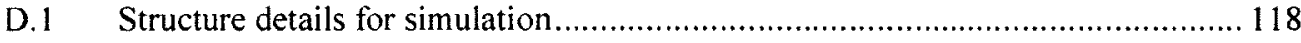

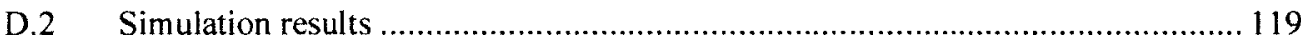

D.3 Comparison and performance evaluation ......................................................... 122

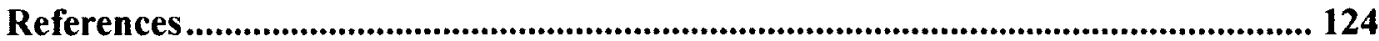




\section{List of Tables}

Table C.1 Periodic overlay simulations. ........................................................ 104

Table C.2 Simulations of a three layer waveguide terminated with a four layer exit section. 104

Table C.3 Simulations using a four layer lead in and a three layer waveguide for the remainder of the structure. 104

Table C.4 Simulations using a $10 \mu \mathrm{m}$ four layer section sandwiched in between matching three layer $20 \mu \mathrm{m}$ sections. 104

Table C.5 Simulations with three layer lead in and lead out waveguide sections....... 105

Table C.6 Simulations with four layer lead in and lead out waveguide sections. ....... 105 Table C.7 Simulations probing convergence of simulation results and structure parameters. 107

Table C.8 Sensor structure simulations to determine the response for mixtures of water and glycerol 110

Table C.9 List of 3-D simulations run including the amount of RAM that simulation of the structure required and the duration of the execution of a corresponding simulation time step. 116 


\section{List of Figures}

Figure 3.1 Four layer waveguide structure showing a thin overlay $\left(t_{2}<t_{3}\right)$ added to a three layer waveguide. 15

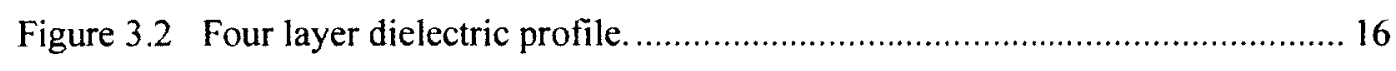
Figure 3.3 Effective index for the 3 and 4 layer modes for increasing overlay refractive index. The 4 layer effective index increases from the 3 layer level and crosses the point where the peak of the mode field amplitude enters the overlay near the desired point of operation. It continues to increase and exceeds the material refractive index of the guiding layer. 21

Figure 3.4 Multimode transition line with multimode operation in the upper left region and single mode operation in the bottom right region of the plot. 22

Figure 3.5 Effective index of modes versus wavelength for the overlay thicknesses of $t_{2}$ $=0.0347 \mu \mathrm{m}$, represented by the solid line, $\mathrm{t}_{2}=0.02222 \mu \mathrm{m}$ and $\mathrm{t}_{2}=0.02845 \mu \mathrm{m}$ represented by the dashed and dash-dotted lines respectively. 23

Figure 3.6 Fundamental mode profile of 3 layer waveguide at a wavelength of $1.78 \mu \mathrm{m}$. (1) 24

Figure 3.7 Profile of second mode in the 4 layer waveguide with a $285 \mathrm{~nm}$ thick overlay at a wavelength of $1.78 \mu \mathrm{m}$ 25

Figure 4.1 Diagram of the waveguide structure with a glass substrate. The overlay segments are of length $d_{1}=243 \mathrm{~nm}$ and the 3 layer waveguide segments are of length $d_{2}$ $=252 \mathrm{~nm}$ providing a pitch of $\Lambda=495 \mathrm{~nm}$. 30

Figure 4.2 Shape of transmission dips for $\Delta \mathrm{n}=0.0$. 33

ix 
Figure 4.3 Shape of transmission dips for $\Delta \mathrm{n}=1.5$ showing Bragg dip location variation for different modeling methods. ............................................................................ 45

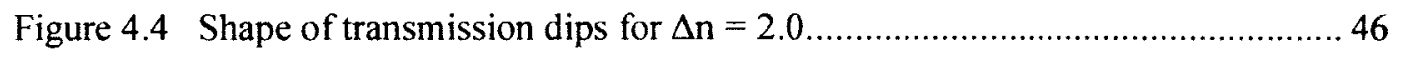

Figure 4.5 Shape of transmission dips for $\Delta \mathrm{n}=1.88$ which represents the use of a silicon

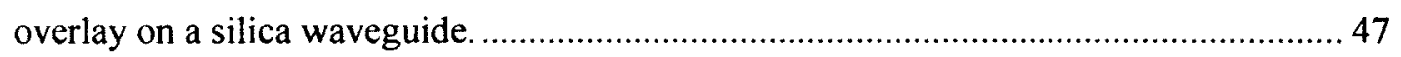

Figure 4.6 Depth of transmission dip versus $\Delta n$ showing that the matrix and perturbation techniques overestimate the strength of the Bragg dip with respect to FDTD. ............... 48

Figure 4.7 Difference in the depth of transmission dip with respect to FDTD versus $\Delta n$

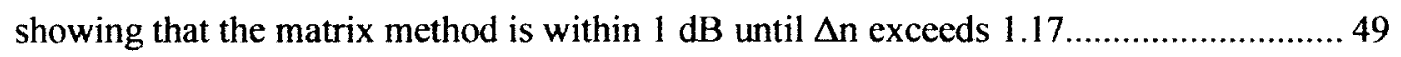

Figure 4.8 Transmission dip wavelength versus increasing $\Delta \mathrm{n}$ showing the matrix method overestimates and the perturbation method underestimates the wavelength with respect to FDTD. The first faint line denotes increase of modal effective index such that the peak enters the overlay near the desired point of operation while the second faint line indicates when the effective index exceeds that of the guiding layer's material refractive index 51

Figure 4.9 Difference in the transmission dip wavelength with respect to FDTD versus $\Delta \mathrm{n}$. 52

Figure 5.1 Standard simulation configuration with source and flux planes indicated. The numerical values on the axis denote the discretized FDTD grid coordinate values of the structure... 55

Figure 5.2 Simulation after 10,000 time steps showing source pulse propagation in 3 layer structure (top) and 4 layer structure (bottom). The three layer structure allows the 
pulse to propagate unchanged while the four layer structure couples specific wavelengths to backward travelling and substrate modes. 55

Figure 5.3 Spectrum of the source pulse (left) next to the spectrum of the propagated pulse (right) showing minimal change of the source pulse when it propagates along the 3

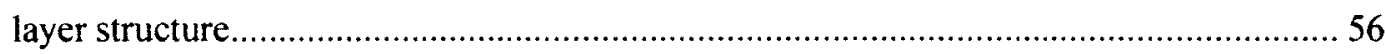

Figure 5.4 Spectrum of the reflection plane flux (left) prior to separating forward and backward travelling components next to the spectrum of the transmission plane flux (right). The reflection flux plane measurement contains both the source flux and the reflected flux while the transmission flux plane measurement contains the transmitted

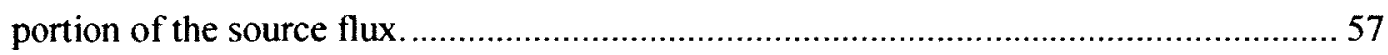

Figure 5.5 Reflectance (left) and transmittance (right) spectra. ...............................57

Figure 5.6 Reflectance (left) and transmittance (right) spectra representing light travel predominantly in line with the overlay. 58

Figure 5.7 Reflectance (left) and transmittance (right) spectra representing light travel predominantly in line with the guiding layer. 58

Figure 5.8 Reflectance (left) and transmittance (right) spectra for structure with $10 \mu \mathrm{m}$ lead in and lead out. 59

Figure 5.9 Reflectance (left) and transmittance (right) spectra for structure with $100 \mu \mathrm{m}$ lead in and lead out. 60

Figure 5.10 Spectra of the sum of reflectance and transmittance spectra for compact simulation domain (left) and for structure with $100 \mu \mathrm{m}$ lead in and lead out (right)...... 60 Figure 5.11 Simulation after 10,000 time steps showing $1.800 \mu \mathrm{m}$ pulse propagation.. 62 Figure 5.12 Simulation after 10,000 time steps showing $1.550 \mu \mathrm{m}$ pulse propagation.. 62 xi 
Figure 5.13 Simulation after 10,000 time steps showing $1.500 \mu \mathrm{m}$ pulse propagation.. 62 Figure 5.14 Simulation including material dispersion to show effect on sensor response at $1.57 \mu \mathrm{m}$. 63

Figure 6.1 Left: four layer structure with $t_{3}$ and $t_{2}$ as the thickness of the thin film waveguide and overlay respectively. Right: corresponding refractive index profile. 67

Figure 6.2 Four and three layer structure TE $\left(E_{Z}\right)$ field profiles respectively, $x$-axes represents position $(\mu \mathrm{m})$ while the $y$-axes represent un-normalized field amplitude. 69 Figure 6.3 Top: $(\mathrm{x}, \mathrm{z})$ plane cut through the grating region of the waveguide configuration. Bottom: Spatial Fourier transform of the grating layer demonstrating the fundamental corresponding to the design wavelength of $1.55 \mu \mathrm{m}$. 71

Figure 6.4 Left: reflectance and Right: transmittance for different grating lengths. The solid line represents the standard simulation with a $20 \mu \mathrm{m}$ length, while the dashed and dotted lines represent 10 and $30 \mu \mathrm{m}$ lengths respectively. 73

Figure 6.5 Reflectance (left) and transmittance (right) for a grating length of $40 \mu \mathrm{m}-$ note the change in vertical axis. 74

Figure 6.6 Left: reflectance and Right: transmittance for gratings with periods designed for different Bragg wavelengths. The solid line represents the standard simulation with $\lambda_{B}$ $=1.5500$, while the dashed and dotted lines represent $\lambda_{B}=1.4091$ and $\lambda_{B}=1.7222$ respectively. 75

Figure 6.7 Left: reflectance and Right: transmittance for different overlay thicknesses. The solid line represents the standard simulation with $\mathrm{t}_{2}=0.0347 \mu \mathrm{m}$, while the dashed and dotted lines represent $t_{2}=0.02222 \mu \mathrm{m}$ and $t_{2}=0.02845 \mu \mathrm{m}$ respectively. 76 
Figure 6.8 Left: reflectance and Right: transmittance for different overlay refractive indices. The solid line represents the standard simulation with $n_{2}=3.480$, while the dashed and dotted lines represent $n_{2}=3.534$ and $n_{2}=3.588$ respectively.

Figure 6.9 Left: change in transmittance. The dashed and dotted lines represent $n_{2}=$ 3.534 and $n_{2}=3.588$ versus the standard simulation respectively. Right: a closer look at transmittance for different overlay refractive indices. The solid line represents the standard simulation with $\mathrm{n}_{2}=3.480$, while the dashed and dotted lines represent $\mathrm{n}_{2}=$ 3.534 and $n_{2}=3.588$ respectively 78

Figure 6.10 Slab waveguide in glass with thin patterned silicon grating overlay. Source, reflection, and transmission planes used in FDTD simulation shown. 81

Figure 6.11 Transmittance (left) and reflectance (right) spectra for device design. 84

Figure 6.12 Reflectance (left) and transmittance (right) spectra for different cladding refractive indices. The solid line represents the pure water trace with $n_{1}=1.315$, while two each of the dashed, dashed and dotted, and dotted lines represent $\mathrm{n}_{1}=1.340,1.365$, $1.390,1.415,1.440$, and 1.465 respectively. Star points on each trace indicate the location of the Bragg wavelength of the grating. 85

Figure 6.13 Change in transmittance. Two each of the dashed, dashed and dotted, and dotted lines represent $n_{1}=1.340,1.365,1.390,1.415,1.440$, and 1.465 versus the $n_{1}=$ 1.315 simulation respectively.

Figure 6.14 Sensor normalized power response for source wavelength of $1556 \mathrm{~nm} \ldots . .88$ Figure A.1 Refractive index versus mass percentages for two sugars, glycerol, and salt showing similar linear trends.

xiii 
Figure B.1 Number of seconds per simulation time step and GB of RAM required for simulations as the number of simulation points increases. 101

Figure C.I Summary of results chart for a sensor structure superstrate refractive index of 1.3541 111

Figure C.2 Summary of results chart for a sensor structure superstrate refractive index of 1.3792 112

Figure C.3 Summary of results chart for a sensor structure superstrate refractive index of 1.4023 113

Figure C.4 Summary of results chart for a sensor structure superstrate refractive index of 1.4264 114

Figure C.5 Summary of results chart for a sensor structure superstrate refractive index of 1.4505 115

Figure D.1 Double taper layer configuration shown by shading in proportion to refractive index. 117

Figure D.2 Over-guide configuration in the SOI platform showing optional removal of a portion of the guiding layer material. 118

Figure D.3 Modal approach results for wavelengths of 1.55 and $1.78 \mu \mathrm{m}$ from top to bottom respectively 119

Figure D.4 FDTD results for wavelengths of 1.55 and $1.78 \mu \mathrm{m}$ from top to bottom respectively. 120

Figure D.5 Three layer output power versus 4 layer superstrate refractive index for lengths of $32.4 \mu \mathrm{m}$ and $262 \mu \mathrm{m}$ 121

xiv 
Figure D.6 FDTD field distribution for double taper structure with the design wavelength of $1.55 \mu \mathrm{m}$. 121

Figure D.7 Modal approach results on left and FDTD results on right for taper coupler with deposited waveguide also tapered and removed above dielectric (top images) and with constant thickness deposited waveguide (bottom images). 


\section{List of Appendices}

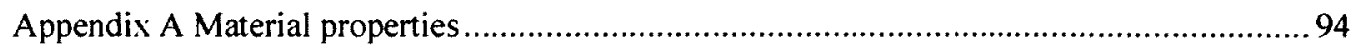

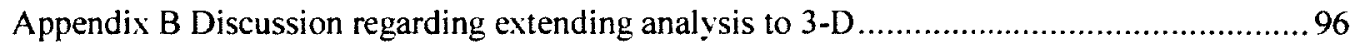

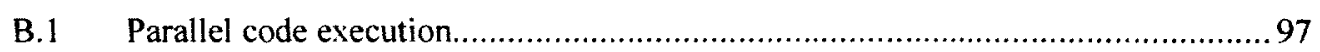

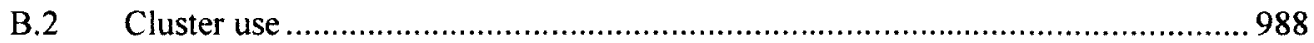

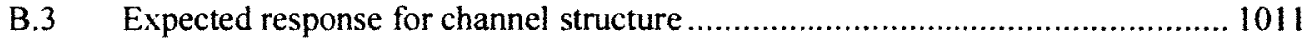

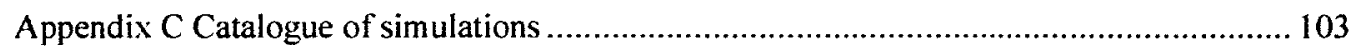

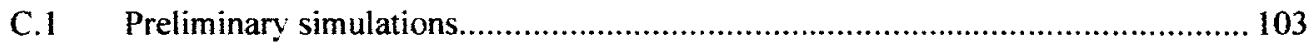

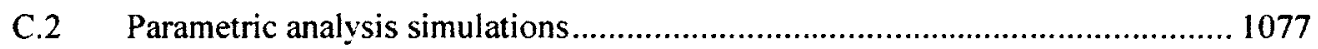

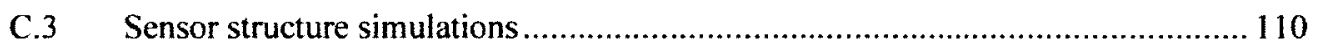

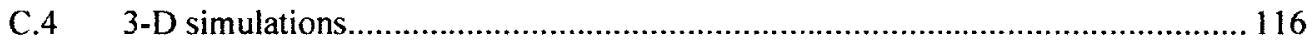

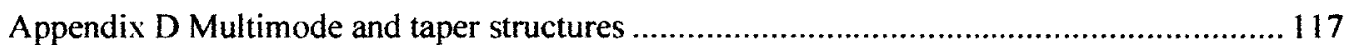

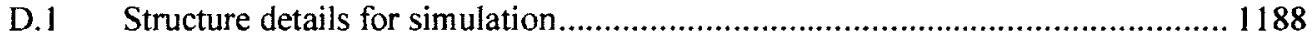

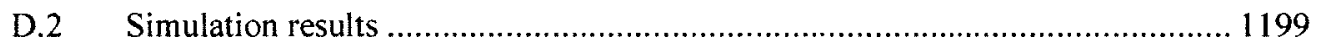

D.3 Comparison and performance evaluation ................................................. 1222 


\section{Introduction}

Silica based planar lightwave circuits (PLCs) have evolved from structures containing Ybranches and directional couplers to structures also containing resonators and filters.[1] In addition, both active and passive device configurations are possible in PLC based glass integrated optics.[2] When interfaced with optical fibre networks, PLCs can demonstrate low insertion losses due to the compatibility of the materials used as waveguides, significant direct waveguide to waveguide mode overlap, and relaxed manufacturing tolerances due to the large mode sizes involved. In general, glass-based integrated optics are relatively large due to the dimensions of the waveguides involved, as determined by the limited dielectric contrast between guiding and substrate-superstrate layers. To achieve more compact and faster integrated optic structures, researchers have turned their attention to substrate materials such as $\mathrm{LiNbO}_{3}$, InP and $\mathrm{Si}$. The silicon on insulator platform (SOI) is of particular interest since it can be processed using conventional silicon nanofabrication techniques.[3] The high dielectric contrast that exists between the silicon layer and the other layers of the SOI platform make it possible to include fast active integrated optic components and compact photonic band gap dielectric configurations such as resonators and waveguides[4] in addition to more compact geometries of the classical glass-based component configurations. Examples of optical components based on the planar technology are the periodically segmented wave mode converter[5] and sub-wavelength periodic structures[6]. To achieve fibre compatibility with reasonably low insertion loss, silicon based devices require a mode transition region. Devices can use the averaged material refractive index approach to decrease the bulk effective index at the coupling interfaces[7]. Another approach to minimize coupling 1 
losses uses an adiabatic thinning of the silicon waveguiding layer, which results in a geometry comparable to strip loaded waveguides[8]. In the near and distant future it is expected that the optical fibre will continue to dominate as the signal carrying medium for optical telecommunications and sensing. The inter-compatibility of active and passive integrated-optic devices and the optical fibre technology used in these environments will remain, requiring low loss, low insertion loss, and fast integrated optic components. These requirements can be met by a glass-based waveguide design as the light guiding region to minimize fibre coupling and insertion losses and then adding a thin high dielectric overlay of patterned silicon at the waveguide superstrate interface to retain the high dielectric contrast and speed of response. The addition of high index material in the vicinity of the waveguide has been shown to permit an increase in optical circuit density and responsivity. For instance, devices in the order of $100 \mu \mathrm{m}$ "propagation" length can be configured into filters and modulators $[9,10]$ or sensors with high resolution[11,12]. In these particular structures, the high dielectric region is applied as a grating overlay, patterned in the propagation direction, in contact with a slab waveguide. In the initial stages of high dielectric clad waveguide structure development, the theoretical analysis phase relies primarily on established techniques such as analytical mode solving, transfer matrix, perturbation, and Finite-Difference-Time-Domain (FDTD). In this thesis, a parametric analysis is conducted on this high dielectric clad waveguide structure and then it is further developed by considering devices that can be built using it.

\subsection{Motivation}

The motivation for this work is to be able to fulfill the requirements for low loss integrated optic components with a glass-based waveguide design as the light guiding 2 
region to minimize fibre coupling and insertion losses and then adding a thin high dielectric overlay of patterned silicon at the waveguide superstrate interface to retain the high dielectric contrast. The use of high speed response capable silicon allows for this work to be extended to building fast active devices. This work will investigate and develop an understanding of this new integrated optic platform for photonic crystals. The effect of adding a high refractive index dielectric overlay to a glass waveguide is fully explored and then applied to optimize device design. This work determines the effect of adding such an overlay.

Many facets of high refractive index dielectric overlay one dimensional photonic crystals are examined. First and foremost are the theoretical approaches which then lead into the modeling and simulation approaches. Then the design of devices on this platform is investigated. Glass waveguide based optical mode sizes are larger than silicon based optical mode sizes. The benefits of the use of glass are low loss transmission in fibre while the benefits of the use of silicon include small device size. The benefit of this work is compact device size without requiring a mode conversion to silicon mode size prior to coupling to the device. Similar fabrication techniques to those of the typical SOI platform can be leveraged. Existing devices that do not require mode conversion to silicon mode dimensions prior to coupling include fibre sensors that can sense fluids by being immersed in them. However, long period gratings in optical fibre have a grating period that ranges from $100 \mu \mathrm{m}$ to $1000 \mu \mathrm{m}$ [13] while the devices described here are entirely within one such period. There exist fibre facet sensors that can use a droplet on the facet instead of requiring immersion.[14-16] The next step smaller is a planar array of sensors onto which a single droplet can be placed. It is this final application, possibly 
incorporated into a handheld sized device, for which the technology herein can be utilized.

3-D simulations are computationally demanding as discussed further in Appendix B. A channel waveguide can be considered as the intersection of two 2-D slabs at 90 degrees to one another.[17] One slab can consider the lateral confinement and the other the vertical confinement of modes. By considering the vertical confinement aspect one can examine the response of guided modes to periodicity in the layers of a waveguide as per the devices developed by this work. It is also possible to use 2-D analysis to solve for the cross-sectional modes of a channel and then subsequently calculate Bragg pitch in the propagation direction to design a structure and then realise it in 3-D where it responds in the expected manner.[18] Thus, the application of 2-D analysis is effective and viable for periodic structures. By using this representation the two-dimensional results for the periodic overlay waveguide structure concepts allow reduced lead time and reduced cost for fabrication of designs.

\subsection{Thesis layout}

Following this introductory chapter, chapter 2 provides the evolution and development of light guiding and periodic structure technology. It then discusses state of the art devices such as sensors. Chapter 3 addresses the theory of guided modes and develops the equations and solutions for the three and four layer waveguide mode field amplitude profiles. These field profiles allow mode overlaps to be calculated. In addition, the equation solutions provide the propagation constants of the modes. Chapter 4 compares three mode propagation modeling approaches. Chapter 5 presents more detail about the FDTD computation engine as well as the results of preliminary computations for a filter 
type device making use of the high refractive index overlay structure. Novel device applications, such as using the filter as a sensor, and the tuning of devices are presented in chapter 6. Chapter 7 summarizes the thesis findings and presents possible directions for future work such as fabrication of the modeled devices. 


\section{Background}

Jean-Daniel Colladon's lectures demonstrated light guiding via total internal reflection in water fountains which is similar to light guiding by glass out of furnaces.[19] Years later, Sir William Henry Bragg reported[20] on the relation of spots exposed on a photographic plate by $\mathrm{x}$-rays to the arrangement of the atoms in a crystal through which they pass. His son, William Lawrence Bragg formalized[21] this providing equation (2.1) leading to their Nobel Prize in Physics.[22]

$$
\mathrm{n} \lambda=2 \Lambda \cos (\theta)
$$

where $\theta$ is the radiation's angle of incidence with respect to the normal of the crystal planes spaced at a pitch of $\Lambda, \mathrm{n}$ is an integer number of wavelengths, and $\lambda$ is the wavelength. In 1963, Amnon Yariv reported on finding optical dielectric waveguiding[23] which would then be further investigated by R. Shubert in thin films as the planar analogue of cylindrical modes in optical fibre.[24] The thorough work contained herein is based on the structure resulting from the combination of these discoveries to provide novel findings.

Planar optical structures continue to be investigated and the simplest of these is the slab waveguide for which the basic theory has been developed and the analytical solution for the three layer structure is widely described[17,25]. The theory has also been extended to address multilayer structures[26]. Slab waveguides are also used for combined photonic and phononic structures. These are known as phoxonic structures for which thin silica plates with silicon pillars can be used and it has been discovered that shorter pillars provide a more complete band gap.[27] Simple dielectric overlays have been considered for sensors[12]. Taking it a step further, the addition of a thin high 6 
refractive index dielectric overlay to slab waveguides has been examined for distributed Bragg reflectors $[9,10]$.

It is possible to analyze the propagation of light through slab waveguide structures using different methods for the purpose of designing grating overlays. The matrix technique relies on segmenting the structure into discrete elements along the propagation direction and computing the transfer properties between segments.[28] The perturbation approach treats the overlay as a perturbation to the waveguide mode's evanescent field.[29] The FDTD simulation relies on a grid discretization of the entire waveguide structure and determination of the time evolution of the electromagnetic fields. [30,31] In the traditional setting in which the overlay region represents a weak modification to the original waveguide structure, the three numerical techniques are in close agreement with regard to the transmission properties of the structure. The numerical results obtained from these three techniques are compared in this work when the overlay greatly alters the waveguide properties. For comparison purposes a representative optical system is employed and described. In order to establish the grounds for the comparison, the basic analysis processes of the matrix, perturbation and FDTD computation techniques are reviewed. Numerical results are then presented as a function of the strength of the overlay index with the FDTD computations being taken as an accurate representation of the expected device response as confirmed through convergence testing.

The numerical FDTD computations determine reflectance and transmittance spectra for a specific set of design parameters representing a target structure. Additional simulations provide results for geometries with varying grating length, grating period, overlay thickness, and refractive index. Fabrication roughness can reduce the sharpness 7 
and strength of a grating dip; these effects of roughness can be compensated for by increased length.[32] While managing sidewall roughness is critical for high refractive index dielectric contrast waveguides, when the mode is predominantly in the underlying layer it is the surface roughness which dominates as the loss mechanism.[33] It is possible to reduce average surface roughness, $R_{a}$, to nearly $1 \mathrm{~nm}$ by using laser crystallization.[34] Existing state of the art devices which use other platforms are discussed next as possible uses of the target structure. Subsequently, the advantages provided by the high dielectric clad platform are considered.

\subsection{State of the art devices}

Planar lightwave circuits have numerous capabilities and can be built using various substrates including glass.[1,2] One of the state of the art applications is fibre-to-thehome/node/etc. (FTTx). Initially the transceivers for this application were built by gluing discrete devices with epoxy to a substrate which then evolved into devices epoxied to a planar waveguide circuit [35] and even direct wafer bonding creating a SOI-InP hybrid[36]. Companies have been looking at $10 \mathrm{~Gb} / \mathrm{s}$ solutions in $\operatorname{InP}[37], 120 \mathrm{~Gb} / \mathrm{s}$ Photonic Integrated Circuits (PICs) [38], and even $1 \mathrm{~Tb} / \mathrm{s}$ wavelength division multiplexed (WDM) devices[39]. In the following subsections, considerations specific to the SOI platform, sensors, and the high refractive index dielectric clad platform will be discussed.

\subsubsection{SOI}

As mentioned earlier, the high dielectric contrast that exists between the silicon layer and the other layers of the SOI platform make it possible to include fast active integrated optic components and compact photonic band gap dielectric configurations such as 8 
resonators and waveguides[4]. It is also of particular interest since it can be processed using conventional silicon nanofabrication techniques.[3] However, getting light in and out of SOI devices can be a challenge not only due to the mode size mismatch but also due to the difficulty with alignment needed for each mass-produced device. Mechanical alignment solutions exist such as v-grooves.[40] Using grating couplers, losses less than

$3 \mathrm{~dB}$ have been reported and can facilitate wafer scale testing[41]; however 3 dimensional positioning is involved. Another challenging situation is coupling to surface plasmon polaritons; strides have been made to address this via end-coupling[42]. The majority of this work focuses on single mode operation. However, section 3.2 includes a quick look at a unique functionality that came about by looking at multimode high refractive index dielectric clad waveguides that function akin to a multimode interferometer (MMI).

\subsubsection{Sensors}

In addition to using SOI as a platform for optical communications, it can be used for sensors. Other platforms for sensors include glass, plasmonic, and polymer waveguides. Sensor interaction can be enhanced via slow light in periodic structures such as gratings. The magnitude of the change in the wavelength of a reflectance peak or transmission dip is expressed per Refractive Index Unit (RIU). Many molecular binding bio-sensing and other label free sensing scenarios utilizing refractive index change detection and representative fluids are considered in Appendix A, Material properties. Sensors can have small footprints and high responsivities such as a proposed $13 \mu \mathrm{m}$ long sensor providing $90 \mathrm{~nm} / \mathrm{RIU}[43]$ and an $8 \mu \mathrm{m}$ long sensor providing $130 \mathrm{~nm} / \mathrm{RIU}$ [44]. The researchers of the former include a comparison to alternative sensing structures. The researchers of the 9 
latter mention a much longer $173 \mu \mathrm{m}$ sensor providing a lower resolution of 33 $\mathrm{nm} / \mathrm{RIU}$ [45] which has better fibre coupling characteristics. Plasmon sensors are found to be able to provide $464 \mathrm{~nm} / \mathrm{RIU}$ using a $10 \mu \mathrm{m}$ sensor with a $10 \mathrm{~dB}$ loss.[46] Polymers also show promise as polymer optical fibre is already used for high fidelity audio system interconnects.[47] In addition, environmental stability has been demonstrated for long lasting electro-optic polymer modulators.[48] Polymers provide the opportunity for electro-optic overlay structures; however, the refractive index contrasts consider large in the polymer field are more than an order of magnitude smaller than that of thin film waveguides and multi-mode considerations must be taken into account.[49,50] Polymers do provide physical design flexibility and adiabatic polymer tapers have been realised.[51] It is also possible to introduce Bragg reflectors in hollow waveguides which can then be infiltrated.[52] The next subsection discusses configuration of the 4-layer structure as a grating sensor and this thesis shows how it can provide a response comparable to the state of the art while using a minimal interaction length.

\subsubsection{Benefits of high refractive index dielectric cladding}

As per the previous chapter, high refractive index dielectric material provides the ability to form periodic optical band gap structures. In addition, with proper design, insertion loss can be reduced between single-mode fibres and diffused channel waveguides.[53] Combining these characteristics into a high refractive index dielectric clad structure provides the benefits of both. The development of overlay gratings $[10,54]$ provides a crucial step in the development of band gap structures using this platform and a clear departure from thin film waveguide relief gratings. Using thinner more confining slab waveguides originally designed as thin film waveguides[55] reduces the physical and 
modal area to be considered by numerical simulations. Some work has been done on the fabrication of this type of structure in thin $\mathrm{Ta}_{2} \mathrm{O}_{5}$ films overlaid on glass waveguides[56] with device lengths on the order of several millimeters. The evolution in this field suggests that the proposed platform of a periodic high refractive index dielectric overlay on a glass waveguide shows promise for state of the art devices. 


\section{Modal theory}

For this work a three layer glass waveguide is modified into a four layer structure by adding a thin high dielectric overlay at the air-waveguide interface. This chapter examines an analytical approach to these multilayer optical structures with the goal of obtaining field profiles and propagation constants. The first section addresses coupling to devices, specifically considering mode overlap and endface losses. The next section presents the details of the waveguide geometry and modal parameters obtained for uniform overlays. Details related to the design of the patterning of the overlay are in section 6.1.1. Spectral discretization[57] was used to confirm the analytical results.

\subsection{Slab Waveguides}

Slab waveguide theory is used to develop an understanding of the structures involved. The analytical concepts developed for one dimension can then be extended to two dimensions. The cross section of a fibre and a channel wave guide can be well matched[53] and representative slab waveguide dimensions can be considered. For the two dimensional case numerical approaches and approximations are used. The basic theory and analytical solution for a three layer structure is widely described $[17,25]$ and has been extended to address multilayer structures with dielectric overlays.[12,26] This enables the modal profiles and propagation constants for the three layer and four layer structures to be determined with high accuracy. However, when the overlay is patterned in the direction of light propagation, the perturbation approach[58] to the waveguide analysis does not apply due to the very high dielectric contrast between waveguide and 
overlay layers. The next chapter examines approaches for high refractive index contrast scenarios.

\subsubsection{Three layer asymmetric guiding}

In an index guided three layer symmetric waveguide the effective index of a guided mode is below that of the core and above that of the surrounding cladding. T he effective index of a guided mode is calculated by dividing the propagation constant by the free space wavenumber. A mode with an effective index below that of the cladding is not guided and power is radiated into the cladding. In the asymmetric waveguide case, with the superstrate index $n_{1}$ being lower than that of the substrate $n_{3}$ which in turn is lower than that of the core layer $n_{2}$, there is an additional possibility. A mode that has an effective index which is higher than the refractive index of the superstrate but lower than that of the substrate is a lossy mode that radiates power into the substrate. The thickness of the core, $t$, also has an effect. If $t$ is increased there is a point at which it becomes multimode, while if $t$ is reduced beyond a certain point even the fundamental mode becomes leaky and thus cut-off. The required thickness, $t$, for the guiding layer is expressed in the asymmetric mode cut-off equations (3.1) and (3.2) for the $m$ th confined TE and TM modes, for integer $m$, respectively for wavelength $\lambda .[29]$

$$
\begin{aligned}
& \left(\frac{t}{\lambda}\right)_{T E}=\frac{1}{2 \pi \sqrt{n_{2}^{2}-n_{3}^{2}}}\left[m \pi+\tan ^{-1}\left(\frac{n_{3}^{2}-n_{1}^{2}}{n_{2}^{2}-n_{3}^{2}}\right)^{1 / 2}\right] \\
& \left(\frac{t}{\lambda}\right)_{T M}=\frac{1}{2 \pi \sqrt{n_{2}^{2}-n_{3}^{2}}}\left[m \pi+\tan ^{-1} \frac{n_{2}^{2}}{n_{1}^{2}}\left(\frac{n_{3}^{2}-n_{1}^{2}}{n_{2}^{2}-n_{3}^{2}}\right)^{1 / 2}\right]
\end{aligned}
$$

The transcendental expressions used to determine the propagation constants and the field profiles of 3 layer structures are well established in the literature. The next section 
discusses the transcendental expressions and field expressions for the lesser known 4 layer structure consisting of a high dielectric clad glass-based slab waveguide.

\subsubsection{Four layer structure}

The addition of a dielectric overlay is shown in figure 3.1, for which the dielectric profile is shown in figure 3.2 with refractive index values chosen as follows. The choice of materials for optical structures is driven by their properties. For optical data transmission the well-established telecommunications band operates around a wavelength of $1.55 \mu \mathrm{m}$ utilizing the optical window of glass between the $1.4 \mu \mathrm{m}$ water absorption peak and 1.7 $\mu \mathrm{m}$ where the lattice absorption of glass becomes significant.[59] The waveguide chosen as the basis for the structure in this work is a glass waveguide. Possible fabrication drove the choice of the parameters of this waveguide. There are many methods of creating glass on glass waveguides with ion migration being a straightforward approach. The facilities in our department are used for integrated electronics and thus the use of salts which can contaminate junctions is frowned upon. An alternative approach is depositing a thin film glass waveguide using established techniques and parameters.[55] This provides a basis structure of a thin film with a thickness of $1 \mu \mathrm{m}$ and a refractive index of 1.6 on a substrate with a refractive index of 1.5 . The desired thin overlay is to have a high refractive index contrast and match the low loss optical window of glass. Silicon is chosen as the material since the optical window is slightly less than the decade of wavelengths from 1 and $10 \mu \mathrm{m}$ which suits the established telecommunications band and its refractive index is 3.48.[25] 


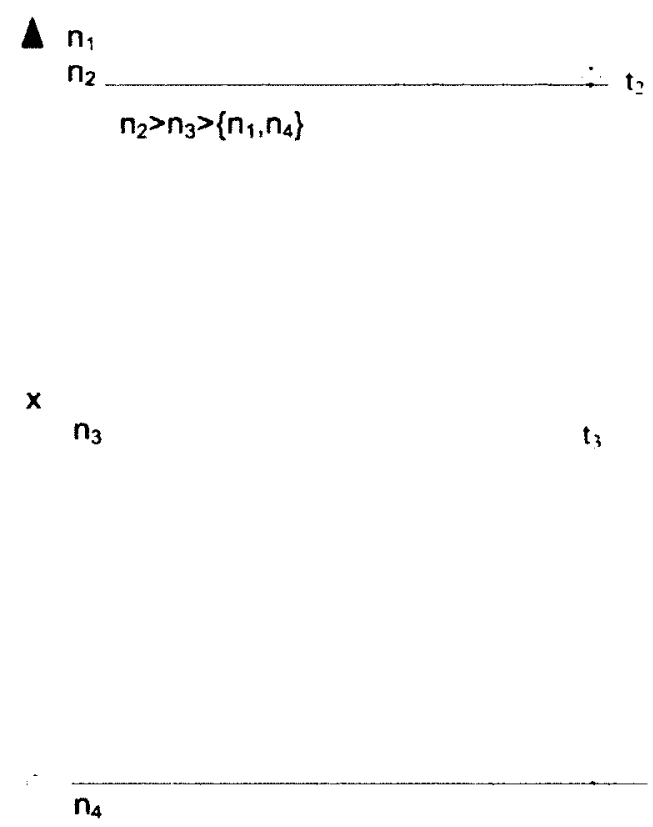

Figure 3.1 Four layer waveguide structure showing a thin overlay $\left(t_{2}<t_{3}\right)$ added to a three layer waveguide.

Note that the thin overlay has a much higher refractive index than the waveguide materials. Much the same as the asymmetric three layer case, there is a point at which the overlay, with thickness $t_{2}$, increases such that the structure becomes multimode. Likewise, the overlay can be thin enough such that it does not support modes on its own. However, since the structure has another layer, a guided mode can still propagate. Multimode operation is not desired; yet the overlay should be sufficiently thick to draw the mode towards it. The desired point of operation is one such that the overlay does not become the guiding layer and the effective index remains below that of the waveguide index. It has been discovered that this desired point can be maintained even when the field peak shifts somewhat into the overlay. 


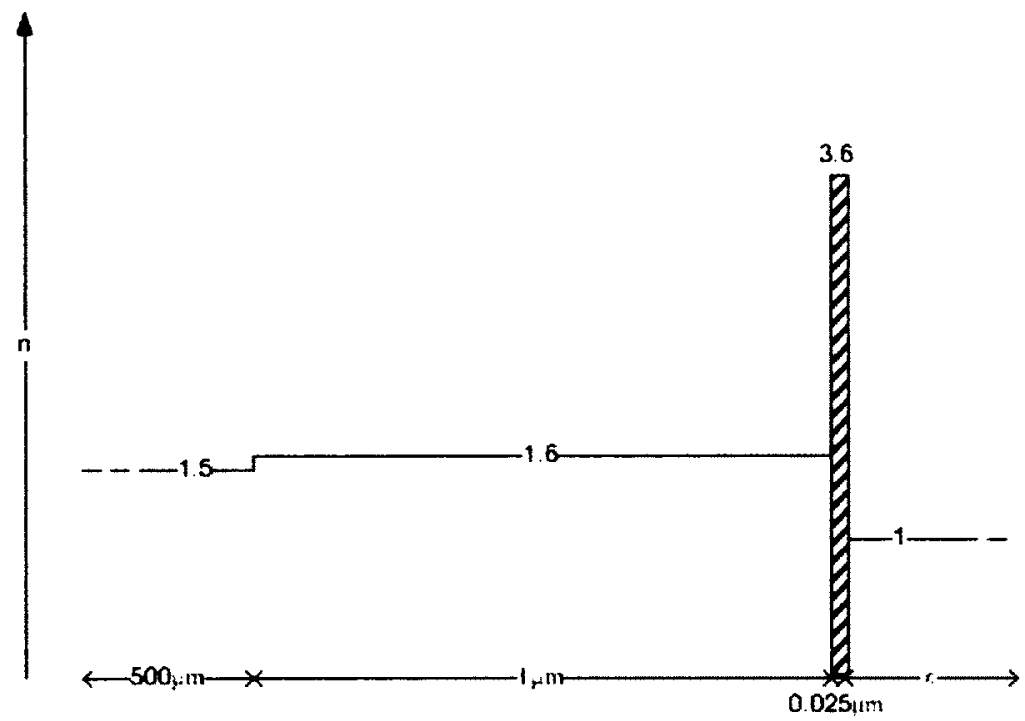

Figure 3.2 Four layer dielectric profile.

\subsubsection{Field equations}

To further investigate the properties of the four layer structure let us consider the applicable wave equation solutions for each region. When the effective index of a mode is above the value of a material refractive index the field of that mode is sinusoidal in that material. When it is below the value it is exponential. For the case when the effective refractive index of guided modes is below both the core guiding layer and the overlay but above that of both the superstrate and substrate, equation (3.3) provides the transverse electric (TE) field profile solution. TE in this work is considered to be when the electric field is linearly polarized and transverse to the direction of propagation while at the same time parallel to the interfaces between the substrate, waveguide, overlay and superstrate. Thus, the TE field profile can be calculated using: 


$$
E_{y}=\left\{\begin{array}{cc}
E_{A} e^{-\sigma\left(x-\left(t_{2}+t_{1}\right)\right)} & x \geq\left(t_{2}+t_{1}\right) \\
E_{B} \sin \left(\kappa_{2} x\right)+E_{C} \cos \left(\kappa_{2} x\right) & \left(t_{2}+t_{1}\right) \geq x \geq t_{2} \\
E_{D} \sin \left(\kappa_{3} x\right)+E_{E} \cos \left(\kappa_{3} x\right) & t_{2} \leq x \leq 0 \\
E_{F} e^{5 x} & x \leq 0
\end{array}\right.
$$

With:

$$
\left\{\begin{array}{l}
\kappa_{2}=\sqrt{\mathrm{k}^{2} \mathrm{n}_{2}^{2}-\beta^{2}} \\
\kappa_{3}=\sqrt{\mathrm{k}^{2} \mathrm{n}_{3}^{2}-\beta^{2}} \\
\sigma=\sqrt{\beta^{2}-\mathrm{k}^{2} \mathrm{n}_{1}^{2}} \\
\xi=\sqrt{\beta^{2}-\mathrm{k}^{2} \mathrm{n}_{4}^{2}}
\end{array}\right.
$$

for the 4 layer waveguide. In the transverse magnetic (TM) case the magnetic field is linearly polarized and transverse to the direction of propagation while at the same time parallel to the interfaces of the dielectric layers. For TM modes, the electric field profile is discontinuous and the field solutions can be calculated by considering the magnetic field equations (3.5) and following calculations very similar to those for the TE.

$$
H_{y}=\left\{\begin{array}{cc}
H_{A} e^{-\sigma\left(x-\left(\left(_{3}+t_{2}\right)\right)\right.} & x \geq\left(t_{3}+t_{2}\right) \\
H_{B} \sin \left(\kappa_{2} x\right)+H_{C} \cos \left(\kappa_{2} x\right) & \left(t_{3}+t_{2}\right) \geq x \geq t_{3} \\
H_{D} \sin \left(\kappa_{3} x\right)+H_{E} \cos \left(\kappa_{3} x\right) & t_{3} \leq x \leq 0 \\
H_{F} e^{5 x} & x \leq 0
\end{array}\right.
$$

When solving the field equations, the relative magnitudes of the adjacent fields are determined. The absolute magnitude is not defined and can be arbitrarily chosen at first. Subsequently, the energy contained with a mode is calculated and the power carried by the mode is set to unity by scaling the amplitude coefficients. This normalization process used in this work simplifies processes such as the calculation of overlap integrals and enables effective comparison of modes from their field profile plots. It is represented by: 


$$
\frac{\beta_{m}}{2 \omega \mu} \int\left[E_{y}(x)\right]^{p} d x=1
$$

\subsubsection{Resulting expressions}

Expressions for the fields can be found using the additional knowledge that the tangential components of $E_{\mathrm{y}}$ and $H_{\mathrm{z}}$ are continuous at the boundaries for the TE modes. By matching the field values of $E_{\mathrm{y}}$ and the derivatives $\frac{d E_{y}}{d x}$ to match field values of $H_{z}$ at the dielectric interfaces it can be found for the TE case that:

$$
\begin{aligned}
& B \kappa_{2} \cos \left(\kappa_{2}\left(t_{3}+t_{2}\right)\right)-C \kappa_{2} \sin \left(\kappa_{2} t_{3}\right) \sin \left(\kappa_{2}\left(t_{3}+t_{1}\right)\right) \\
& +\sigma B \sin \left(\kappa_{2}\left(t_{3}+t_{2}\right)\right)+\sigma \sin \left(\kappa_{2} t_{3}\right) \cos \left(\kappa_{2}\left(t_{3}+t_{2}\right)\right)=0
\end{aligned}
$$

For:

$$
B=\xi_{2} \sin \left(\kappa_{3} t_{3}\right)+\kappa_{2} \kappa_{3} \cos \left(\kappa_{3} t_{3}\right)-C \cos \left(\kappa_{2} t_{3}\right)
$$

and:

$$
\begin{aligned}
C= & -\xi \kappa_{3} \cos \left(\kappa_{3} t_{3}\right) \sin \left(\kappa_{2} t_{3}\right)+\kappa_{3} \kappa_{3} \sin \left(\kappa_{3} t_{3}\right) \sin \left(\kappa_{2} t_{3}\right) \\
& +\xi \sin \left(\kappa_{3} t_{3}\right) \kappa_{2} \cos \left(\kappa_{2} t_{3}\right)+\kappa_{3} \cos \left(\kappa_{3} t_{3}\right) \kappa_{2} \cos \left(\kappa_{2} t_{3}\right)
\end{aligned}
$$

Which provides the same result for $t_{2}=0$ as solving the 3 layer transcendental equation:

$$
\kappa_{2} t_{3}=m \pi+\arctan \left(\frac{\xi}{\kappa_{2}}\right)+\arctan \left(\frac{\sigma}{\kappa_{2}}\right)
$$


For the case when the effective index of the mode exceeds that of the core guiding layer it has an exponential wave solution in that region:

$$
E_{y}=\left\{\begin{array}{cc}
E_{A} e^{\left.-\kappa_{1}\left(x-t_{3}+r_{2}\right)\right)} & x \geq\left(t_{3}+t_{2}\right) \\
E_{B} \sin \left(\kappa_{2}\left(x-t_{3}\right)\right)+E_{C} \cos \left(\kappa_{2}\left(x-t_{3}\right)\right) & \left(t_{3}+t_{2}\right) \geq x \geq t_{3} \\
E_{D} e^{\kappa_{3} x}+E_{E} e^{-x_{3} x} & t_{3} \leq x \leq 0 \\
E_{F} e^{\lambda_{1} x} & x<0
\end{array}\right.
$$

Thus for the 4 layer waveguide:

$$
\left\{\begin{array}{l}
\kappa_{1}=\sqrt{\beta^{2}-\mathrm{k}^{2} \mathrm{n}_{1}^{2}} \\
\kappa_{2}=\sqrt{\mathrm{k}^{2} \mathrm{n}_{2}^{2}-\beta^{2}} \\
\kappa_{3}=\sqrt{\beta^{2}-\mathrm{k}^{2} \mathrm{n}_{3}^{2}} \\
\kappa_{4}=\sqrt{\beta^{2}-\mathrm{k}^{2} \mathrm{n}_{4}^{2}}
\end{array}\right.
$$

Following some calculation the solution is:

$$
A \kappa_{1} e_{+} t_{3}+B \kappa_{3} e_{+} t_{3}=-A \kappa_{1} e_{-} t_{3}+B \kappa_{3} e_{-} t_{3}
$$

with:

$$
e_{+} t_{3}=\left(\frac{1}{2}+\frac{\kappa_{4}}{2 \kappa_{3}}\right) e^{\kappa_{3} t_{3}} \text { and } e_{-} t_{2}=\left(\frac{1}{2}-\frac{\kappa_{4}}{2 \kappa_{3}}\right) e^{-\kappa_{3} t_{3}}
$$

as well as:

$$
A=k_{2}\left[c t_{2}-\frac{k_{2}}{k_{1}} s t_{2}\right] \text { and } \mathfrak{B}=\left[k_{1} s t_{2}+k_{2} c t_{2}\right]
$$

where

$$
c t_{2}=\cos \left(k_{2} t_{2}\right) \text { and } s t_{2}=\sin \left(k_{2} t_{2}\right) .
$$

\subsubsection{Structure design}

Using the expressions for the fields it was discovered that the peak of the field amplitude transitions into the overlay prior to the effective index exceeding that of the guiding glass layer. Figure 3.3 shows the plot of the effective mode index in the 3-layer and the 4-layer 
regions of the structure. The values are determined using a standard mode solving technique. $[17,25,26]$ The effective index in the 3-layer region is constant as it does not depend on the presence of the overlay. In the 4-layer region, the mode's effective index is a function of overlay index and increases as $\Delta \mathrm{n}$ increases where $\Delta \mathrm{n}$ is the increase in index of the overlay with respect to the waveguide layer. For sufficiently large $\Delta \mathrm{n}$, the effective index crosses above the 1.6 (waveguide) index value and the grating layer supports the mode with exponential decay in the waveguide layer. Also of interest in this figure is the plot of the peak transition line.[60] For modal effective index values below this line, the peak field amplitude of the mode is located within the glass waveguide region. For effective index values above this line, the mode's peak field amplitude is located in the overlay region. Examination of the 4-layer effective index trace indicates that for a selected range of $n_{2}$ between 3.27 and 3.50 , the mode has an effective index in the glass range and peak in the overlay. This unique property is unavailable in low index bounded waveguides. 


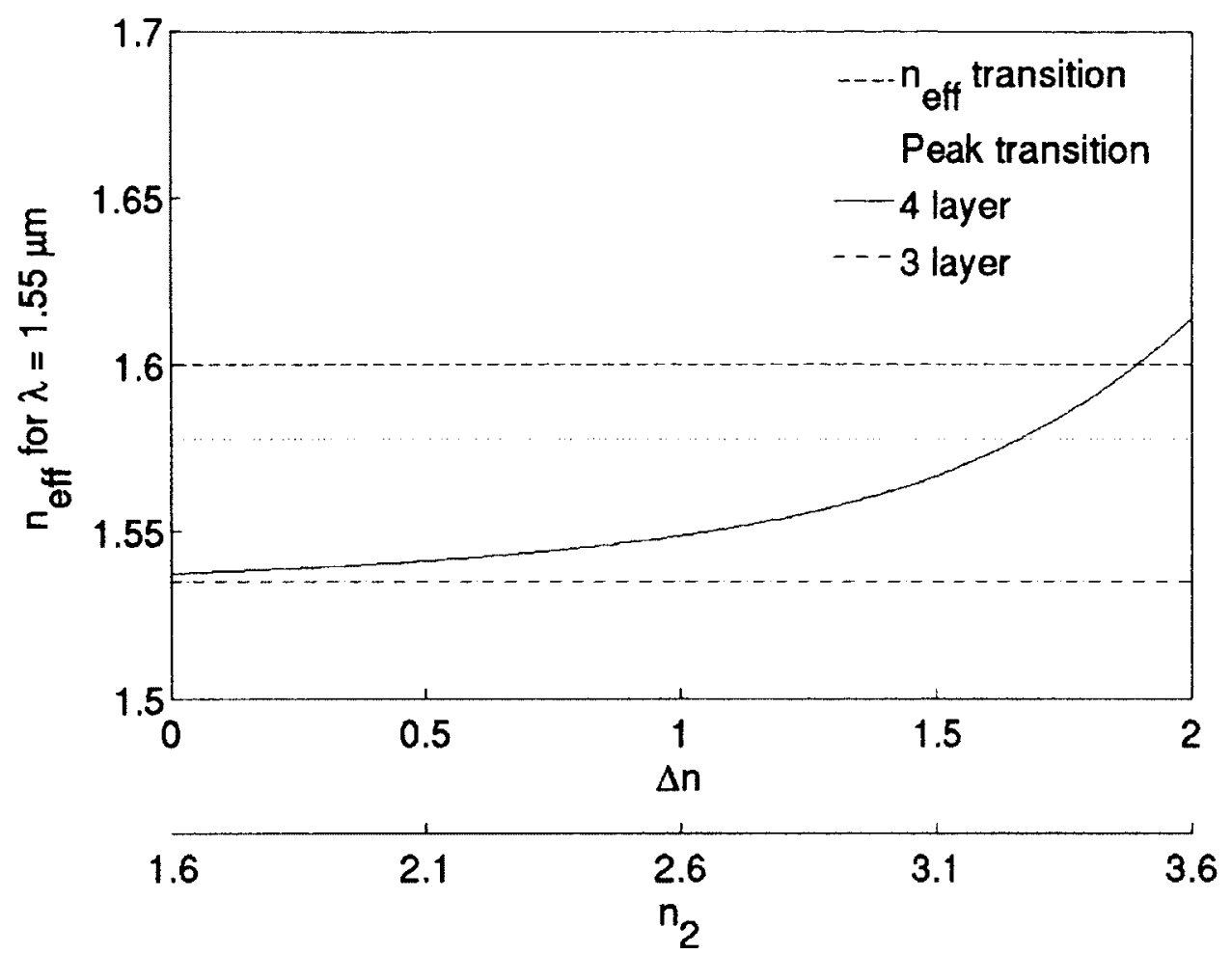

Figure 3.3 Effective index for the 3 and 4 layer modes for increasing overlay refractive index. The 4 layer effective index increases from the 3 layer level and crosses the point where the peak of the mode field amplitude enters the overlay near the desired point of operation. It continues to increase and exceeds the material refractive index of the guiding layer.

Subsection 3.1.2 discussed the choice of materials based on their material properties and most calculations are completed for wavelengths from $1.4 \mu \mathrm{m}$ to $2.0 \mu \mathrm{m}$ with the plot range from $1.7 \mu \mathrm{m}$ to $2.0 \mu \mathrm{m}$ primarily to show properties of structures that when utilized are wavelength shifted into the transparency window. In addition to the material properties the structure provides a characteristic point of operation where the peak of the mode is within the overlay yet the effective index still has a value below the refractive index of the guiding layer. This provides input as to what thickness of overlay 
to use. An addition factor as to what thickness to choose is shown in figure 3.4 where the point at which the structure becomes multimode is denoted with multimode operation in the upper left region and single mode operation in the bottom right region of the plot showing that for the chosen thickness operation is single mode for the wavelengths of interest.

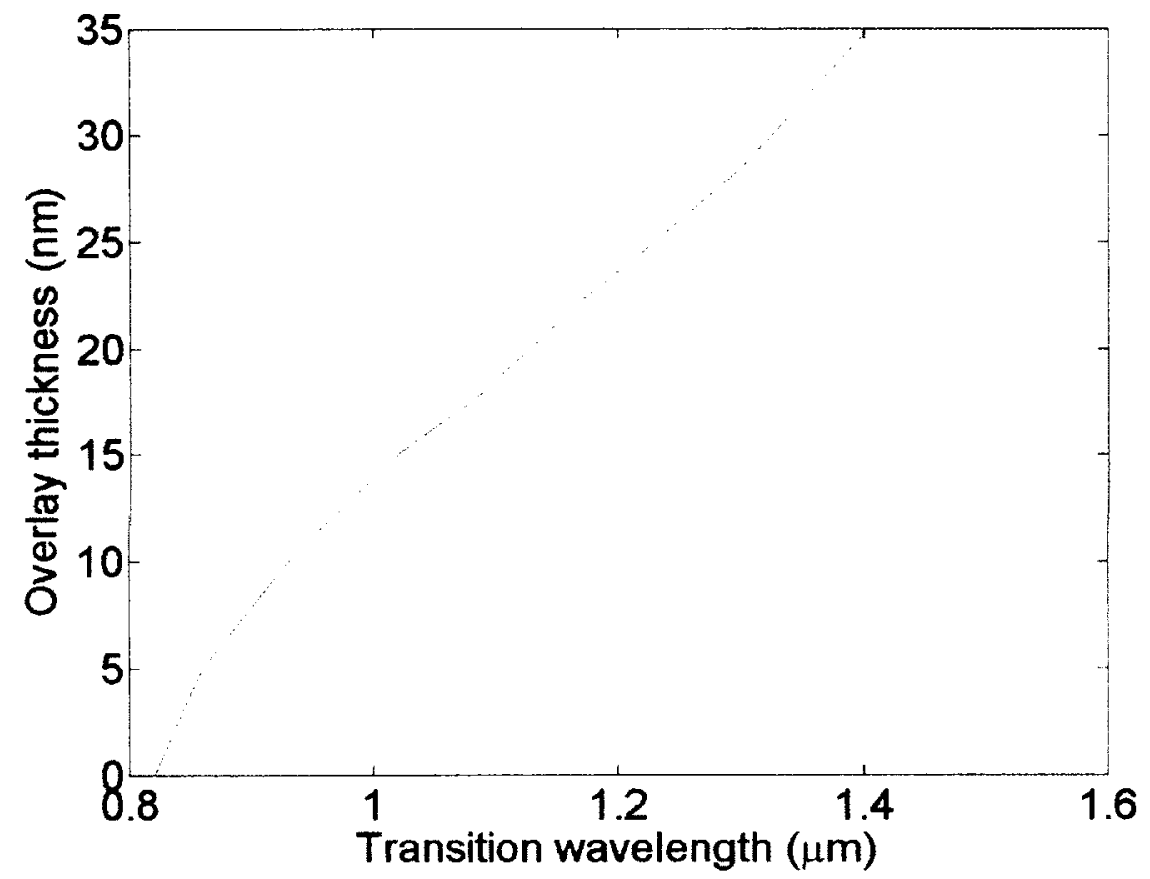

Figure 3.4 Multimode transition line with multimode operation in the upper left region and single mode operation in the bottom right region of the plot.

Furthermore the effective index of modes versus wavelength is plotted in figure 3.5 for the overlay thicknesses that are further explored in the parametric analysis in section 6.1. It is evident that in the wavelength range of interest that the effective index is very near that of the refractive index of the wave guiding material refractive index. 


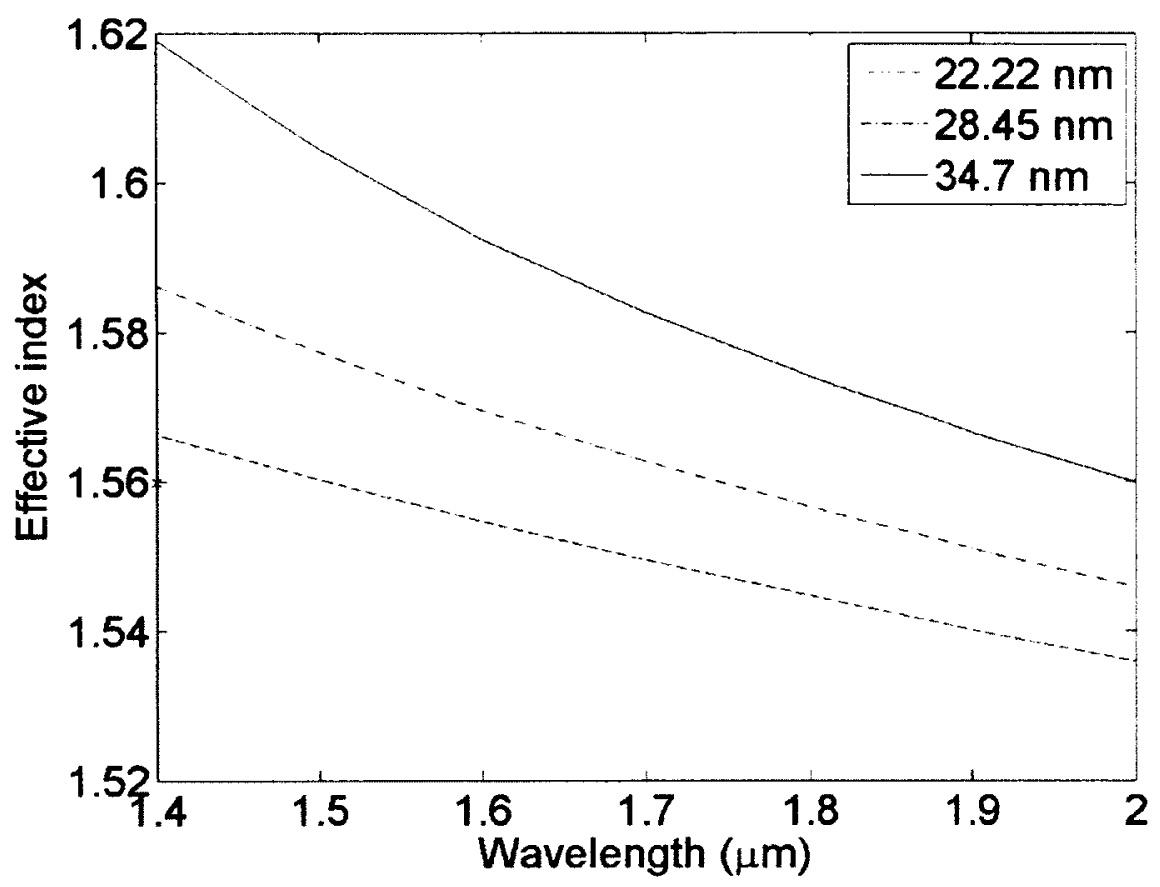

Figure 3.5 Effective index of modes versus wavelength for the overlay thicknesses of $t_{2}=0.0347 \mu \mathrm{m}$, represented by the solid line, $t_{2}=0.02222 \mu \mathrm{m}$ and $t_{2}=0.02845 \mu \mathrm{m}$ represented by the dashed and dash-dotted lines respectively.

3.2 Multimode device considerations for thin high index dielectric overlay slab waveguides

This section introduces the novel use of multimode thin high dielectric overlay slab waveguide structures for integrated optics as published and presented in concert with this work.[60,61] The coupling of light to the platform and its capability for devices are covered. In order to explore the structures, modal modeling methods are used alongside FDTD. Two interesting features have been discovered. They are related to structures with somewhat thicker waveguide dimensions such that the structures are multimode.

\subsubsection{Design and objective}


The first multimode overlay structure feature is related to the calculation of the field profile peak crossing into the overlay prior to the effective index. The feature discovered in addition to the peak crossing is a configuration, which is not possible with a single mode structure, where the effective index of a 3 layer mode and a 4 layer mode are the same. This results in the field profile of the fundamental mode of the 3 layer waveguide overlapping exactly in the substrate and waveguide regions with the field profile of the second mode when the overlay is added as shown in Figures 3.6 and 3.7 respectively.

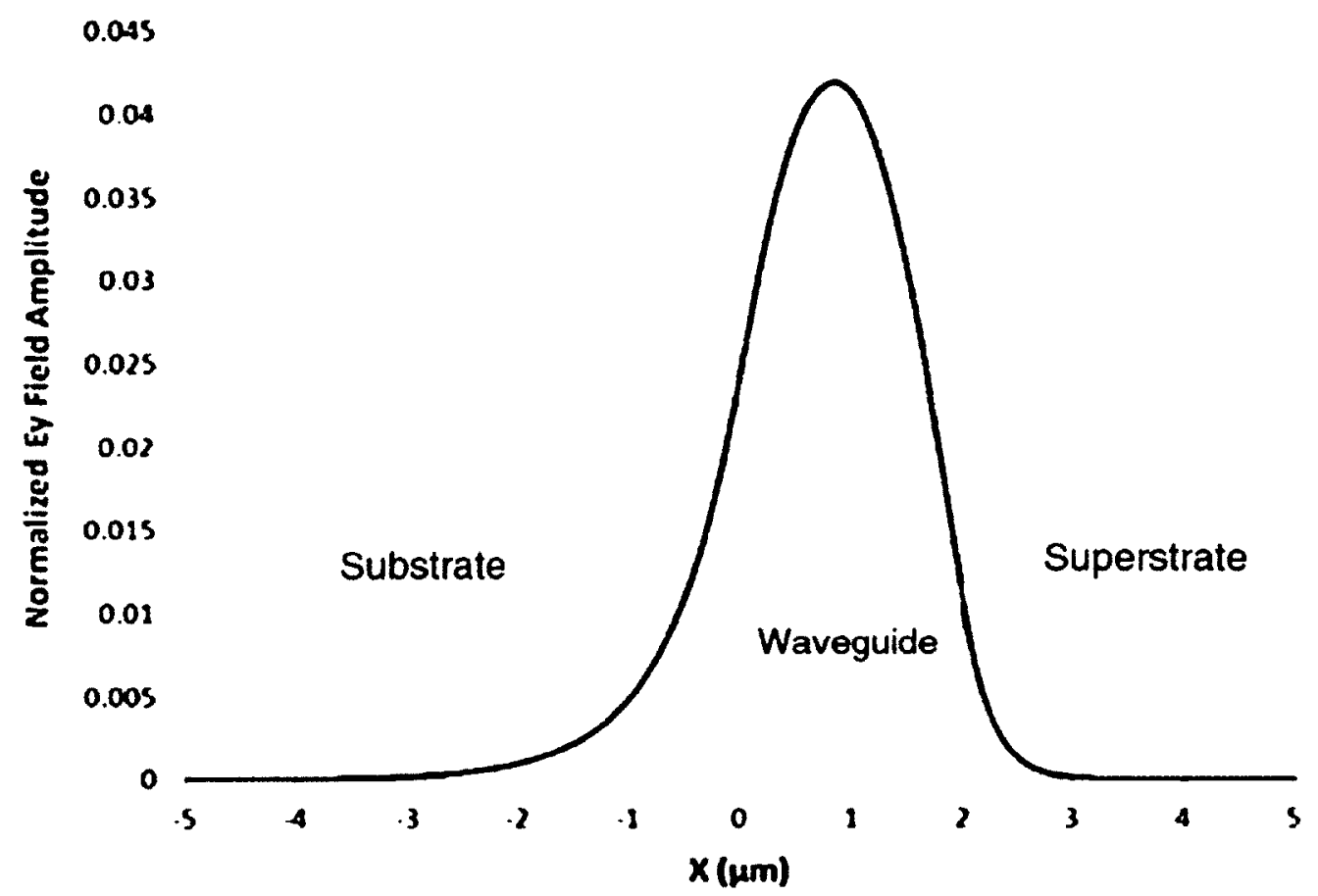

Figure 3.6 Fundamental mode profile of 3 layer waveguide at a wavelength of $1.78 \mu \mathrm{m}$. 


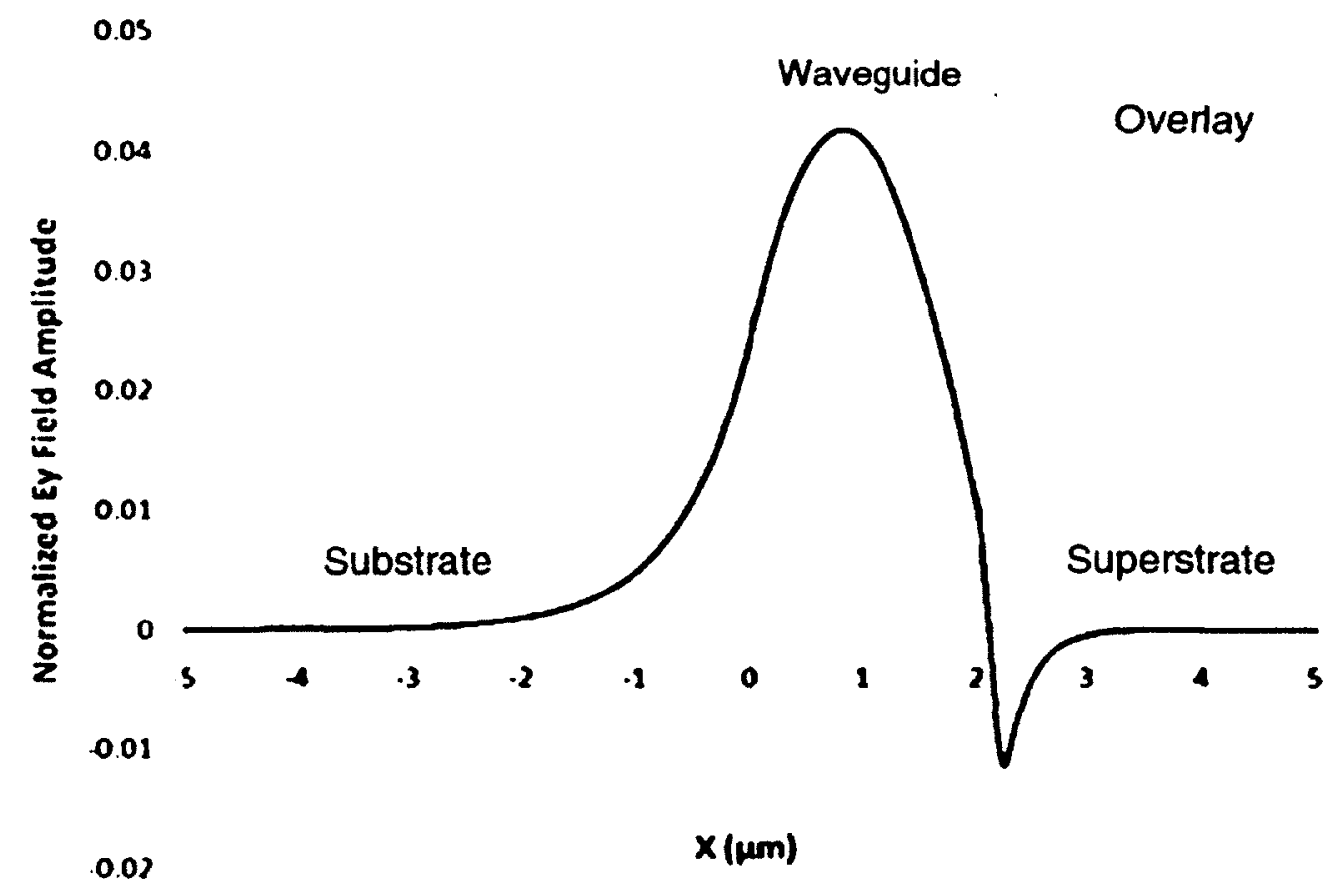

Figure 3.7 Profile of second mode in the 4 layer waveguide with a $285 \mathrm{~nm}$ thick overlay at a wavelength of $1.78 \mu \mathrm{m}$.

The second novel feature is that the MMI behaviour of the 4 layer waveguide structure between two 3 layer waveguide sections provides layer specific coupling control. For instance for straight through coupling the input port is the wave guide layer prior to the structure and the output port is the wave guide layer after the structure. The structure consists of the un-patterned overlay section where it behaves as an MMI-like device where the interference pattern's peak moves from the guiding layer input port into the overlay and back into the guiding layer for the output port for the design wavelength. For light off the design wavelength the coupling to the output port is reduced until the next harmonic constructive interference condition for the output port is again reached. The previously mentioned coupling of the 3 layer mode to a matched 4 layer mode provides 25 
throughput for a case when this has not necessarily been reached and thus the structure does not always behave as a true MMI with equally excited modes. In this manner this structure has a filter effect that can be used in a similar manner to the single mode structure with the periodic overlay. This filter effect is also sensitive to the region above the overlay and thus the structure can also be used as a sensor.

\subsection{Coupling}

Coupling takes place from the input fibre to the waveguide and also from the homogenous region of the waveguide to the structured region. A similar coupling path in reverse takes place for light exiting the device. The critical coupling to optimize is from the waveguide to the patterned overlay.

\subsubsection{Overlap integrals}

To consider the efficiency of coupling not only into the waveguides themselves from a fibre but also from a clad region to an unclad (or photonic crystal) region the overlap integrals are considered using equation $(3.17) .[53,62]$

$$
\eta=\frac{\left.\int_{-\infty-\infty}^{\infty} \iint \Psi_{1}(x, y) \Psi_{2}^{*}(x, y)\right|^{2} d x d y}{\int_{-\infty}^{\infty} \int_{-\infty}^{\infty} \Psi_{1}(x, y) \Psi_{1}^{*}(x, y) d x d y \int_{-\infty}^{\infty} \iint_{-\infty}^{\infty} \Psi_{2}(x, y) \Psi_{2}^{*}(x, y) d x d y}
$$

The fibre is butt coupled to the cleaved facet of the waveguide. The lateral accuracy for efficient coupling can be manipulated to $\pm 1 \mu \mathrm{m}$. There is a mode mismatch between the fibre and the waveguide resulting in about a $3 \mathrm{~dB}$ insertion loss in the case of $\mathrm{LiNbO}_{3} .[63]$ Using the Gaussian electric field amplitude distribution:

$$
E(\rho)=e^{\left(\frac{2 \rho \ln 0.5)^{2}}{W_{\text {FIMS }}}\right)^{2}}
$$


it is possible to approximate the two dimensional cross-sectional distribution of a field. The field will be approximated for an optical fibre, a nano wire, and a channel waveguide. For this approximation, a FWHM beam width of $8 \mu \mathrm{m}$ is used for an optical fibre, $0.5 \mu \mathrm{m}$ is used for a nano wire, and $1.8 \mu \mathrm{m}$ is used for the channel waveguide. The modal overlap integral[64]:

$$
P_{z}=\sum_{\mu} \mathrm{a}_{\mu} \mathrm{a}_{\mu}^{*}\left[1 / 4 \iint_{\mu}\left\{\vec{E}_{\mu} \times \vec{H}_{\mu}^{*}+\vec{E}_{\mu}^{*} \times \vec{H}_{\mu}\right\} \cdot \vec{k} d x d y\right]
$$

with the coefficients:

$$
\begin{aligned}
a_{v} & =\int_{-\infty}^{\infty} E_{y} E_{v} d x / \int_{-\infty}^{\infty} E_{v}^{2} d x \\
& =\left\langle E_{y}, E_{v y}\right\rangle /\left\langle E_{v}, E_{v y}\right\rangle
\end{aligned}
$$

can be used to calculate the expected power to be coupled to a butt coupled nano wire to be $1.6 \%$ and an order of magnitude higher at $18 \%$ for the channel waveguide. These results can now be compared to the coupling efficiency to the actual mode shape in the planar waveguide. A planar waveguide is infinite in lateral extent. However, for the purposes of this calculation, the overlap integral is calculated for a lateral extent which is $+/-16 \mu \mathrm{m}$ from the centre of the optical fibre. Beyond this distance the coupling from the fibre to the planar waveguide is insignificant. The coupling is found to be $14 \%$ which is comparable to that found using the approximation for the channel waveguide. The coupling can be improved by adiabatic tapers and by the design of the channel waveguide.

\subsubsection{Endface losses}

Reflection can take into account the losses from the facet of a device but there is also a cavity formed by sequential facets, or those with some separation. For this work we use the following definitions regarding light flow at an interface: 
The reflection and transmission coefficients $\mathrm{r}$ and $\mathrm{t}$ are ratios of the complex amplitudes. The power reflectance $R$ and transmittance $T$ are defined as the ratios of power flow (along a direction normal to the boundary) of the reflected and transmitted waves to that of the incident wave.[65]

It is possible to calculate the loss due to Fresnel reflection. The output of light from medium 1 (air) to medium 3 (guide) experiences Fresnel reflection following[65]

$$
\operatorname{loss}(d B)=-10 \log \left(1-\left(\frac{n_{1}-n_{3}}{n_{1}+n_{3}}\right)^{2}\right)
$$

which provides a loss factor of less than a quarter of a decibel for such an interface. Sequential facets form a Fabry-Perot cavity for which the effective cavity length is dependent on the medium's refractive index. Index matching gel can be used to minimize both reflection losses and reduce the effective cavity length. For the glass waveguide it would be possible to match the indices to eliminate this loss. 


\section{Modeling techniques}

The intention of this chapter is to compare three of the most common numerical analysis techniques (Matrix, Perturbation, FDTD) with the aim of showing which is the most suited for efficient and accurate modeling of the high dielectric clad slab waveguides. The reason this must be done is that typical techniques do not take into account the high refractive index dielectric contrast of the overlay. All un-patterned overlay waveguide configurations are analyzed by solving Maxwell's equations for the 3 and 4 layer structures while patterned overlay waveguide configurations are analyzed using the FDTD computation technique. Further detail of the FDTD technique is provided in chapter 5 .

\subsection{Patterned overlay system}

High dielectric overlays patterned as gratings on glass waveguides show promise for enhanced waveguides, filters, and sensors. The optical system is shown in figure 4.1 and is designed to operate around the central optical telecommunications wavelength of 1.55 $\mu \mathrm{m}$. The out of the page direction is considered to be infinite and uniform. The waveguide has an index of refraction $n_{3}=1.600$ and a thickness $t_{3}=1 \mu \mathrm{m}$ placed on a substrate of refractive index $n_{4}=1.500$, infinite in extent below the waveguide. The superstrate has a refractive index $n_{1}=1.000$ and extends to infinity above the waveguide. In a periodic manner the waveguide is modified into a 4 layer structure by adding a thin high index dielectric overlay at the air-waveguide interface. The thickness of the overlay segments is $t_{2}=34.7 \mathrm{~nm}$; when made of silicon, it has an index $n_{2}=3.480$. This combination of thickness and index is chosen to be representative of a high refractive 
index overlay yet be thin enough such that it does not become an independent waveguide nor make it possible for the structure to be multimode. The diagram in figure 4.1 shows only 6 of the $\mathrm{N}=40$ segments considered in the propagation direction. The grating segments each have a pitch of $\Lambda=495 \mathrm{~nm}$, with $d_{1}=243 \mathrm{~nm}$ for the "silicon" and $d_{2}=$ $252 \mathrm{~nm}$ for the "air" portions of the grating segment. All propagating light is taken to be polarized in the out of the page direction (TE). The silicon and air lengths of the grating segment were chosen such that they are each a quarter wavelength long for the single mode supported in the waveguide structure at $\lambda_{0}=1.55 \mu \mathrm{m}$ with an overlay of silicon.

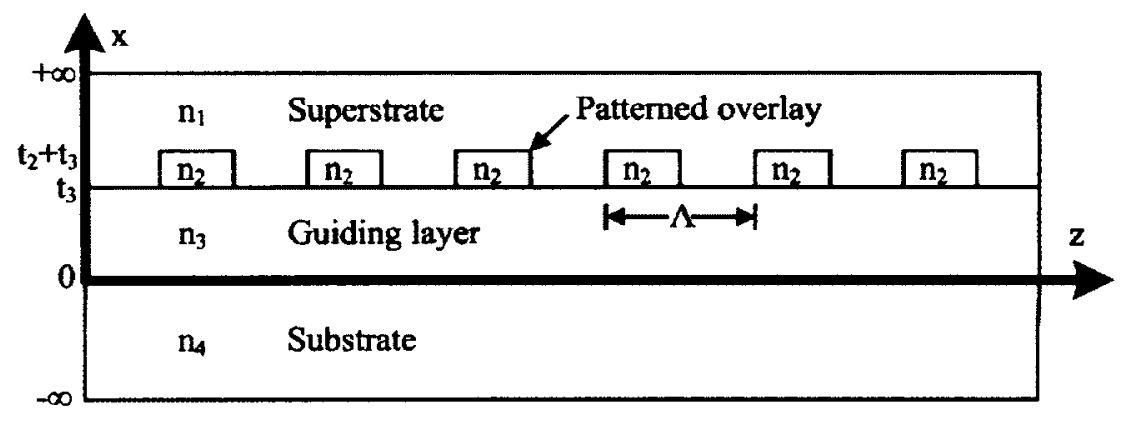

Figure 4.1 Diagram of the waveguide structure with a glass substrate. The overlay segments are of length $d_{1}=243 \mathrm{~nm}$ and the 3 layer waveguide segments are of length $d_{2}=252 \mathrm{~nm}$ providing a pitch of $\Lambda=495 \mathrm{~nm}$.

Chapter 3 detailed solving for the modal field amplitudes and the detailed analysis of this structure as a sensor and waveguide is presented in chapter 6 . In the computations required for the comparison of the theoretical techniques, only the index value of the overlay is changed and all other parameters are kept constant. When the grating index matches the waveguide index, the three numerical techniques are known to be in good 
agreement and will serve as a base reference to examine the divergence of the techniques as the index is increased above that of silicon at $1.55 \mu \mathrm{m}$.

\subsection{Matrix technique}

This technique comprises of representing the optical system as a single matrix equation relating the coupling between forward and backward travelling wave amplitudes at a particular wavelength which can be respectively represented by a 2 element column matrix at a specific point. Solving this equation for a range of wavelengths provides the reflection and transmittance spectra data which can then be plotted and compared to other techniques. The matrix technique is easily applied to a waveguide with an overlay grating for the full 40 period long structure described in section 1 and shown in figure 4.1. The structure consists of 4 layer waveguide regions where the overlay is present alternating with 3 layer regions. Each of these regions can be modeled as a one dimensional segment of uniform refractive index with a value equal to the effective index of the mode that propagates in that segment.

The matrix representing the entire structure is built by multiplying matrices which represent each individual period of the grating. The grating matrices are in turn the product of 4 building block matrices. Two distinct four-element square building block matrices are required, interface and translation. The interface matrix relates field amplitudes at the boundary of two different refractive indices. The fields are taken as normally incident and the transmission and reflection coefficients are calculated by considering the effective indices of the modes in the 3 layer and 4 layer sections as bulk indices.[65] The matrix elements are structured to include the coefficients such that 
multiple reflections are represented at a particular interface.[28] The propagation matrix accounts for the phase change that occurs for a distance $d$ travelled in a uniform index region. Therefore, for each grating period, there are two interface matrices and two propagation matrices. The relation between field components on either side of one grating period is shown in equation (4.1)

$$
\left[\begin{array}{l}
c_{i} \\
b_{i}
\end{array}\right]=\left[\begin{array}{cc}
\frac{1}{t_{x 12}} & -\frac{r_{x 21}}{t_{x 12}} \\
\frac{r_{x 12}}{t_{x 12}} & \frac{t_{x 12} t_{x 21}-r_{x 12} r_{x 21}}{t_{x 12}}
\end{array}\right]\left[\begin{array}{cc}
e^{j k d_{2}} & 0 \\
0 & e^{-j k d_{2}}
\end{array}\right]\left[\begin{array}{cc}
\frac{1}{t_{x 21}} & -\frac{r_{x 12}}{t_{x 21}} \\
\frac{r_{x 21}}{t_{x 21}} & \frac{t_{x 21} t_{x 12}-r_{x 21} r_{x 12}}{t_{x 21}}
\end{array}\right]\left[\begin{array}{cc}
e^{j k d_{1}} & 0 \\
0 & e^{-j k d_{1}}
\end{array}\right]\left[\begin{array}{c}
c_{i+1} \\
b_{i+1}
\end{array}\right]
$$

where $c_{i}$ is the incident, $c_{i+1}$ is the transmitted, $b_{i}$ is the backward reflected, and $b_{i+1}$ is the backward incident electric field wave amplitude along the propagation axis.

Modeling each sequential optical element in the propagation direction as individual matrices allows the system matrix $M$ in equation (4.2) to be calculated through a matrix multiplication process.

$$
\begin{aligned}
& M= \\
& {\left[\begin{array}{cc}
\frac{e^{j k\left(d_{1}+d_{2}\right)}-r_{x 21}{ }^{2} e^{j k\left(d_{1}-d_{2}\right)}}{t_{x 12} t_{x 21}} & \frac{-r_{x 12} e^{j k\left(d_{2}-d_{1}\right)}-r_{x 21}\left(t_{x 21} t_{x 12}-r_{x 21} r_{x 12}\right) e^{-j k\left(d_{2}+d_{1}\right)}}{t_{x 12} t_{x 21}} \\
\frac{r_{x 12} e^{j k\left(d_{1}+d_{2}\right)}+\left(t_{x 12} t_{x 21}-r_{x 12} r_{x 21}\right) r_{x 21} e^{j k\left(d_{1}-d_{2}\right)}}{t_{x 12} t_{x 21}} & \frac{-r_{x 12}{ }^{2} e^{j k\left(d_{2}-d_{1}\right)}+\left(t_{x 12} t_{x 21}-r_{x 12} r_{x 21}\right)^{2} e^{-j k\left(d_{2}+d_{1}\right)}}{t_{x 12} t_{x 21}}
\end{array}\right]}
\end{aligned}
$$

The overall matrix equation is represented by equation (4.3)

$$
\left[\begin{array}{l}
c_{1} \\
b_{1}
\end{array}\right]=M^{N}\left[\begin{array}{l}
c_{N} \\
b_{N}
\end{array}\right]
$$


where $b_{1}$ is the reflected electric field amplitude and $c_{X}$ is the total transmitted electric field amplitude which would be detected for the entire structure. In this overall case, the only input source is the incident wave $c_{1}$ thus $b_{N}$, which represents light incident on the output port of the structure, is set to zero.

The results achieved through the matrix approach are depicted in figure 4.2 (top) for an overlay refractive index of $1.6(\Delta \mathrm{n}=0.0)$. The transmittance spectrum shows a main $0.04 \mathrm{~dB}$ transmission dip which has small side lobes as per a Bragg response[66]. The light that is not transmitted is reflected through coupling to the backwards propagating mode. The Bragg dip is at a wavelength of $1.52 \mu \mathrm{m}$, which corresponds to the pitch of the grating for an overlay refractive index of 1.6.
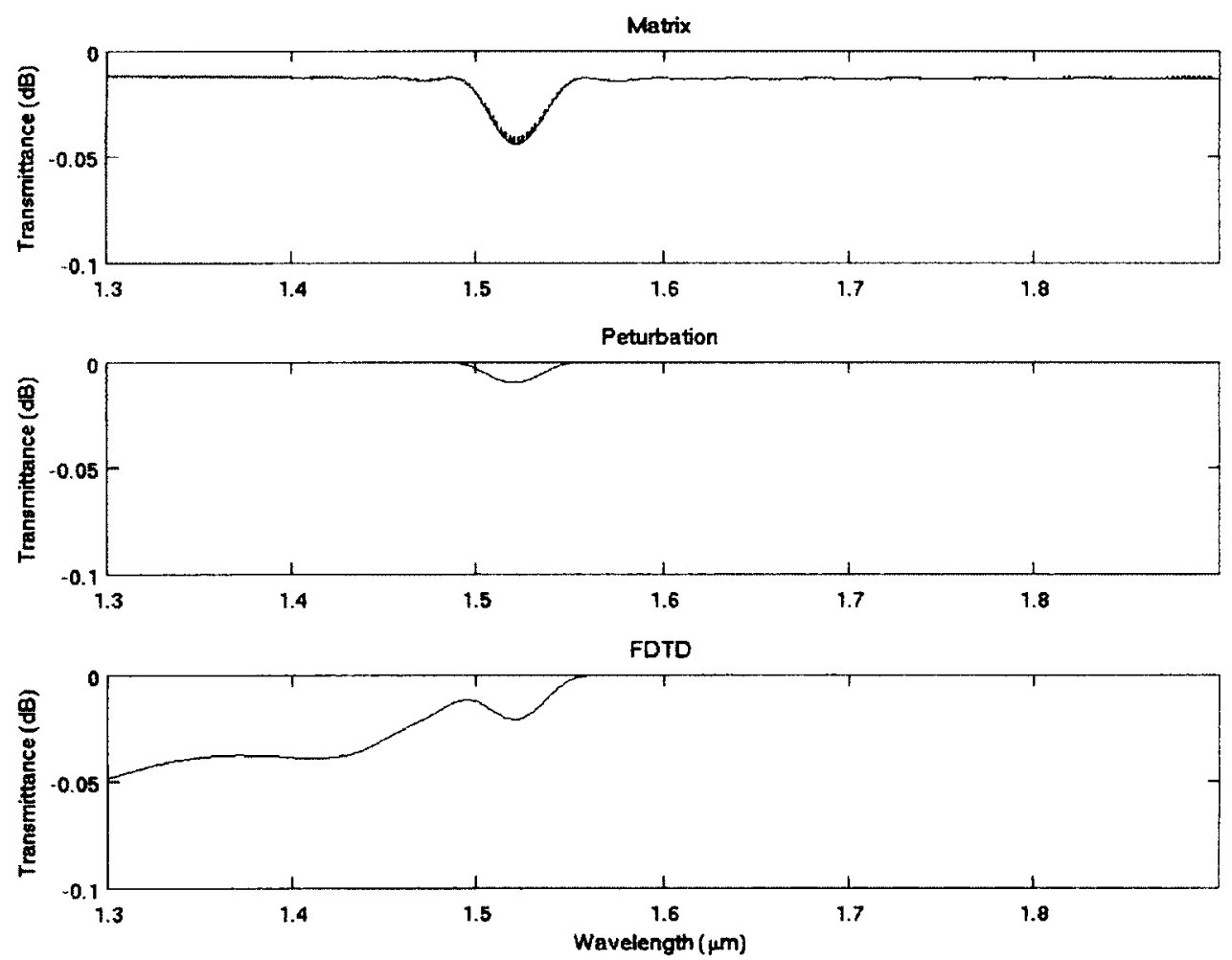

Figure 4.2 Shape of transmission dips for $\Delta \mathrm{n}=\mathbf{0 . 0}$.

33 


\subsection{Perturbation approach}

This approach is based on treating a modification to a waveguide as a perturbation to an otherwise planar and uniform waveguide structure. Considering the comparison optical system of figure 4.1 , the modification consists of the periodic overlay which loads the 3 layer waveguide profile in the propagation direction. This perturbation causes energy from the forward travelling mode to be coupled to the backward traveling mode with the amplitude coupling coefficient calculated through expressions obtained from [67] and computed using the numerical process of [29]. The formulation assumes that the mode is reasonably confined such "that operation is sufficiently above propagation cutoff' [29] and the effective index of the mode is near that of the guiding layer. Then the paraxial approximation can be used to simplify the expression of the field profile when calculating the coupling coefficient. Other additional assumptions regarding the structure are made and those of particular interest will be discussed. Most perturbation approaches and their related approximations assume that the perturbation is considered to be relatively small. Equation (4.4) shows the electric field vector of the electromagnetic wave expressed as an expansion in the normal modes of the unperturbed dielectric structure.[29]

$\sum_{k}\left[\frac{d^{2}}{d z^{2}} A_{k}-2 i \beta_{k} \frac{d}{d z} A_{k}\right] \mathbf{E}_{k}(x, y) e^{-i \beta_{k^{z}}}=-\omega^{2} \mu \sum_{l} \Delta \varepsilon(x, y, z) A_{l} \mathbf{E}_{l}(x, y) e^{-i \beta_{l} z}$

When the dielectric perturbation is weak, it is found that (4.5) holds[29]

$$
\left|\frac{d^{2}}{d z^{2}} A_{k}\right| \ll\left|\beta_{k} \frac{d}{d z} A_{k}\right|
$$

allowing the second order derivative term in (4.4) to be dropped via the parabolic approximation.[29] An additional simplification is the assumption that the effective index 
of the mode is well represented in the perturbed region by the unperturbed value. This is embodied in the approximation generally made[29] where:

$$
\Delta \varepsilon(x, z)=\Delta \varepsilon(x) \frac{f(z)+1}{2}
$$

contains $f(z)$ as a square wave for which the Fourier expansion is used and only the first term of the expansion is retained. The input source is considered to be at the beginning of the alternating segments. The detector is considered to be at the end of 40 periods where the portion of the light that is not transmitted due to coupling to the backwards propagating mode is calculated. The result of the perturbation approach is plotted for an overlay refractive index of 1.6 as shown in figure 4.2. (middle). The $0.01 \mathrm{~dB}$ Bragg dip is at a wavelength of $1.52 \mu \mathrm{m}$ for an overlay refractive index of 1.6 .

\subsection{FDTD Simulation}

Finite-Difference-Time-Domain (FDTD) simulation is a widely used numerical approach to modeling electromagnetic structures. This section begins with the basics of the FDTD method[68,69] followed by results used for comparison with the other methods. The treatment of dispersion is an important consideration. Dispersion is the variation of the phase velocity of the light propagating in different modes or different wavelengths. It has an influence on group velocity, amplitude, and shape. Chromatic dispersion will occur due to the variation of the dimensions of the structure relative to the wavelength. It will also occur due to the variation of the refractive index of materials with respect to the wavelength. The material dispersion subset of chromatic dispersion is not significant for the narrow range of wavelengths used for the structures considered. Dispersion will also occur in multimode situations since each mode can experience a different effective index 35 
resulting in variation of propagation velocity. This includes different order modes as well as modes of differing polarizations.[59] The effect of additional dispersion introduced by numerical simulation when using central finite difference approximations for spatial functions can be dispensed by ensuring sufficient wavelength resolution and the use of Gaussian sources with FDTD.[70] The materials used for this work are considered for the most part to be linear, non-dispersive, and non-magnetic. Material dispersion effects are indeed investigated to confirm that they are not significant as is convergence of results for increasing resolution. Thus, the basic equations to be solved across the problem domain are the time-dependent Maxwell's vectorial curl equations for propagation in materials with a unity relative magnetic permeability:

$$
\begin{aligned}
& \frac{\partial E}{\partial t}=\frac{1}{\varepsilon_{r} \varepsilon_{0}} \nabla \times \boldsymbol{H} \\
& \frac{\partial \boldsymbol{H}}{\partial t}=-\frac{1}{\mu_{0}} \nabla \times \boldsymbol{E}
\end{aligned}
$$

These equations can be expanded into six coupled partial differential equations as follows:

$$
\begin{gathered}
\frac{\partial E_{x}}{\partial t}=\frac{1}{\varepsilon_{r} \varepsilon_{0}}\left(\frac{\partial H_{z}}{\partial y}-\frac{\partial H_{y}}{\partial z}\right) \\
\frac{\partial E_{y}}{\partial t}=\frac{1}{\varepsilon_{r} \varepsilon_{0}}\left(\frac{\partial H_{x}}{\partial z}-\frac{\partial H_{z}}{\partial x}\right) \\
\frac{\partial E_{z}}{\partial t}=\frac{1}{\varepsilon_{r} \varepsilon_{0}}\left(\frac{\partial H_{y}}{\partial x}-\frac{\partial H_{x}}{\partial y}\right) \\
\frac{\partial H_{x}}{\partial t}=\frac{1}{\mu_{0}}\left(\frac{\partial E_{y}}{\partial z}-\frac{\partial E_{z}}{\partial y}\right) \\
\frac{\partial H_{y}}{\partial t}=\frac{1}{\mu_{0}}\left(\frac{\delta E_{z}}{\delta x}-\frac{\delta E_{x}}{\delta z}\right)
\end{gathered}
$$




$$
\frac{\partial H_{z}}{\partial t}=\frac{1}{\mu_{0}}\left(\frac{\partial E_{x}}{\partial y}-\frac{\partial E_{y}}{\partial x}\right)
$$

These equations describe the six field quantities that are involved in three dimensional simulations. The $z$-axis is taken to be positive in the direction of propagation and the $x$ axis to be positive in the direction from the substrate to the superstrate. These axes describe the 2-D simulation plane with the $y$-axis positive out of the plane. In the case of two dimensional simulations it is possible to choose either the transverse electric (TE) mode of $E_{y}, H_{z}$, and $H_{x}$, or the transverse magnetic (TM) mode of $H_{y}, E_{z}$, and $E_{x}$. In order to avoid having the magnitudes of $\mathrm{E}$ and $\mathrm{H}$ differ by several orders of magnitude the following change of variables is made:

$$
\tilde{E}=\sqrt{\frac{\varepsilon_{0}}{\mu_{0}}} E
$$

Considering the TE linearly polarized field reduces the field equations to:

$$
\begin{gathered}
\frac{\partial \tilde{E}_{y}(t)}{\partial t}=\frac{1}{\varepsilon_{r} \sqrt{\varepsilon_{0} \mu_{0}}}\left(\frac{\partial H_{x}(t)}{\partial z}-\frac{\partial H_{z}(t)}{\partial x}\right) \\
\frac{\partial H_{z}(t)}{\partial t}=-\frac{1}{\sqrt{\varepsilon_{0} \mu_{0}}} \frac{\partial \tilde{E}_{y}(t)}{\partial x} \\
\frac{\partial H_{x}(t)}{\partial t}=\frac{1}{\sqrt{\varepsilon_{0} \mu_{0}}} \frac{\partial \tilde{E}_{y}(t)}{\partial z}
\end{gathered}
$$

These field equations are changed to finite difference equations for computation with boundary conditions[71]. The central finite difference approximations are:

$$
\frac{\tilde{E}_{y}^{n+\frac{1}{2}}(i, j)-\tilde{E}_{y}^{n-\frac{1}{2}}(i, j)}{\Delta t}=\frac{1}{\varepsilon_{r} \sqrt{\varepsilon_{0} \mu_{0}}}\left(\frac{H_{x}^{n}\left(i+\frac{1}{2}, j\right)-H_{x}^{n}\left(i-\frac{1}{2}, j\right)}{\Delta z}-\frac{H_{z}^{n}\left(i, j+\frac{1}{2}\right)-H_{z}^{n}\left(i, j-\frac{1}{2}\right)}{\Delta x}\right)
$$




$$
\begin{aligned}
& \frac{H_{z}^{n+1}\left(i, j+\frac{1}{2}\right)-H_{z}^{n}\left(i, j+\frac{1}{2}\right)}{\Delta t}=-\frac{1}{\sqrt{\varepsilon_{0} \mu_{0}}} \frac{\tilde{E}_{y}^{n+\frac{1}{2}}(i, j+1)-\tilde{E}_{y}^{n+\frac{1}{2}}(i, j)}{\Delta x} \\
& \frac{H_{x}^{n+1}\left(i+\frac{1}{2}, j\right)-H_{x}^{n}\left(i+\frac{1}{2}, j\right)}{\Delta t}=\frac{1}{\sqrt{\varepsilon_{0} \mu_{0}}} \frac{\tilde{E}_{y}^{n+\frac{1}{2}}(i+1, j)-\tilde{E}_{y}^{n+\frac{1}{2}}(i, j)}{\Delta z}
\end{aligned}
$$

The separation between neighbouring elements $i$ and $i+1$ is $\Delta z$ and for between $j$ and $j+1$ it is $\Delta x$. The superscripts represent the time in integer multiples of the time interval $\Delta t$. To describe the dielectric structure in the problem space $\varepsilon_{r}(i, j)$ can be used in place of $\varepsilon_{r}$. The problem region is discretized in the $x-z$ plane, taking into account resolving both the spatial dielectric values as well as the spatial wavelength range of the source field in the dielectric. The separation between neighbouring elements is tested in order to ensure convergence for decreasing values. Ten elements per wavelength in the structure under test is a starting point but in this work the small features of the silicon overlay require additional elements in order to be resolved. The Courant condition:

$$
\Delta t \leq \frac{\Delta z}{c_{0}}
$$

embodies the concept that the time interval shall be no greater than the time it takes light to cross an element. Using a Courant factor of:

$$
s=\frac{\Delta t}{\Delta z \sqrt{\varepsilon_{0} \mu_{0}}}=\frac{1}{2}
$$

also allows for anti-aliased and stable solutions for up to the 3 dimensional simulations. Incorporating this factor into the approximations and rearranging them allows them to be used iteratively in discretized computational code as follows:

$$
\tilde{E}_{y}^{n+\frac{1}{2}}(i, j)=\tilde{E}_{y}^{n-\frac{1}{2}}(i, j)+\frac{\left(H_{x}^{n}\left(i+\frac{1}{2}, j\right)-H_{x}^{n}\left(i-\frac{1}{2}, j\right)\right)-\left(H_{z}^{n}\left(i, j+\frac{1}{2}\right)-H_{z}^{n}\left(i, j-\frac{1}{2}\right)\right)}{2 \varepsilon_{r}}
$$




$$
\begin{aligned}
& H_{z}^{n+1}\left(i, j+\frac{1}{2}\right)=H_{z}^{n}\left(i, j+\frac{1}{2}\right)-\frac{\tilde{E}_{y}^{n+\frac{1}{2}}(i, j+1)-\tilde{E}_{y}^{n+\frac{1}{2}}(i, j)}{2} \\
& H_{x}^{n+1}\left(i+\frac{1}{2}, j\right)=H_{x}^{n}\left(i+\frac{1}{2}, j\right)+\frac{\widetilde{E}_{y}^{n+\frac{1}{2}}(i+1, j)-\widetilde{E}_{y}^{n+\frac{1}{2}}(i, j)}{2}
\end{aligned}
$$

When defining the boundaries of the numerical simulation it is necessary to consider their impact on the problem space. In calculating the field values the surrounding values are used while at the boundary one must consider what values should be used. If the boundary represents a reflection plane then it is straightforward to implement by enforcing the appropriate rules at the boundaries. If the boundary represents the border of a periodic structure it is also straightforward to assign values based on the edge of the boundary matching the values of its partner boundary. However, if boundary of the problem space simply represents the border of the area of interest while in reality the material would continue towards infinity it is important to not allow the discontinuity to create interference. In this case, the energy of a propagating field travelling towards the boundary should be absorbed. Since in most cases all sources are within the problem space, all fields at the boundary are travelling outwards. Both the case of a corner of the problem space and a field travelling along a boundary are a bit more involved. Extra care is taken to create an absorbing boundary buffer that is several units in thickness, matched to the dielectric value of the problem space and is attenuating. This more complex absorbing boundary condition $(\mathrm{ABC})$ is called a perfectly matched layer (PML). Different thicknesses and types of PMLs were tested at different distances from the problem space to ensure that those used would not affect results. The materials used for the actual structure are isotropic but in implementing the boundary conditions 39 
anisotropic capable software can improve accuracy by reducing error from step changes at interfaces.[30]

To provide source excitation to this model the corresponding value can be added to the appropriate discretized points at the appropriate time steps. The input source consists of a short time duration pulse with a profile and field components matched to those for the 3-layer geometry obtained by solving the field equation for the field polarized in the y direction, $E_{y}(x)$. The multi-wavelength source is defined as the discretetime derivative of a Gaussian,

$$
\left.E_{y}(x, t)=E_{y}(x)(-i \omega)^{-1} \frac{\partial}{\partial t} \exp \left(-i \omega t-\left(t-t_{0}\right)^{2}\right) / 2 w^{2}\right)
$$

In the case of a steady sinusoidal source the resulting propagating fields of a structure can be analysed once the simulation reaches a steady state. Instead of doing such an analysis it is possible to use a pulse and examine the flux through planes of interest for the structure versus that in baseline simulation. To calculate the Fourier transform of $E(t)$ for a frequency, $f$, the following equation can be used:

$$
E(f)=\int_{0}^{\tau} E(t) e^{-j 2 \pi f t} d t
$$

Since an integral is a sum, this could be computed for frequencies of interest, at points of interest, during the simulation without storing the complete field for each time step until the end of the simulation. The specific FDTD[30] formulation can be extended to 3-D with extensive additional storage and computational requirements for additional as outlined in Appendix B.

In order to solve for the transmittance and the reflectance of the comparison structure (figure 4.1), the FDTD simulation process requires 3 layer input and output 40 
waveguides to be attached to the grated waveguide structure described in section 4.1 . The optical source and back reflection flux plane are placed within the input segment. The source consists of a short time domain pulse with a Gaussian spectral width centered at $1.55 \mu \mathrm{m}$. Its amplitude profile is matched to the fundamental mode of the 3 layer input waveguide segment at $1.55 \mu \mathrm{m}$. The 3 layer output segment contains the transmission flux plane. The computational domain is surrounded by a $1 \mu \mathrm{m}$ perfectly matched layer (PML) with matched dielectric values to reduce reflections from the grid border. The simulation domain is discretized in a square grid at a resolution of 160 points per $\mu \mathrm{m}$. Based on the size and discretized properties of the structure, 120,000 iterations are sufficient to ensure convergence and that the optical pulse has fully vacated the computation domain. For normalization purposes, the transmitted flux of the simulated grating structures are normalized to the transmitted flux of the structure without an overlay.

The transmittance spectrum for this method is shown in figure 4.2 (bottom) for an overlay refractive index of $1.6(\Delta \mathrm{n}=0.0)$. The $0.02 \mathrm{~dB}$ Bragg dip is at a wavelength of $1.52 \mu \mathrm{m}$ for an overlay refractive index of 1.6. Above a wavelength of $1.60 \mu \mathrm{m}$ there is nearly full transmission since the guided mode does not match the Bragg condition resulting in high throughput. On the lower wavelength side, the transmission is low and is due to the excitation of a continuum of both reflected and radiated substrate modes. [72] These modes are excited via the phase matching that takes place from the guided mode to the diffracted radiation modes with counterpropagating fields beginning at a wavelength slightly shorter than the Bragg wavelength since their wavevector is long enough to 
incorporate some angle from the guided mode vector when phase matched to the grating.[73]

\subsection{Discussion and technique selection}

In this section we examine the effect of gradually increasing the overlay index of refraction from 1.6 (waveguide) to 3.6 (slightly higher than that of silicon) and compare the spectral response obtained from the three techniques. Figure 4.2 shows the result when the overlay grating has the same index of refraction as the waveguide region, $\Delta n=$

0.0. All three methods are in agreement as to the location of the Bragg dip at a wavelength of $1.52 \mu \mathrm{m}$ for $\Delta \mathrm{n}=0.0$. However, the depths of the dips differ due to the extremely thin overlay grating layer. In addition, the low wavelength side of the spectral response of the first two techniques are significantly different than that returned from the FDTD computation.[9] The matrix technique does not account for the transfer of energy into the superstrate or substrate that may either be radiated away or re-coupled in subsequent grating segments into the waveguide region. The matrix technique can be expanded into a mode propagation method using additional overlap integrals between the various substrate and superstrate modes present. However this requires the use of matrices of order much greater than 2 and periodic boundary conditions must be considered to normalize the radiated modal profiles. These added complexities, required to bring matrix results in line with FDTD results offset the simplicity of the matrix approach and render it unattractive. However, the matrix approach has long been considered to be more accurate than coupled mode theory for large index differences in distributed-feedback waveguide devices.[74] Modern versions of the matrix method have been commercialized and continue to be used for semiconductor sources.[75] The 42 
perturbation approach can also be extended to include interactions between additional modes, however, the inclusion of additional coupled modes increases the technique's complexity since the dominant mode in the perturbed region is taken as a sum over all modes of the unperturbed structure. The coupling to a finite number of modes can be completed however in the case of a continuum of modes or when the bounds of the modes are undefined even a known vendor of modern commercial software for this purpose has begun to offer FDTD solutions.[76] The goal of this work is to take the fundamental form of a technique and apply it. The finding is that the matrix and perturbation methods require added complexity to provide accurate results while the FDTD method results simply needs to be checked for convergence.

Figure 4.3 shows the shape of transmittance spectra for the three computational techniques when $\Delta \mathrm{n}=1.5$ providing an overlay refractive index of 3.1. This figure shows that the locations of the Bragg dip predicted by the three techniques can have a significant difference. Figure 4.3 , top, for the matrix based results, shows a Bragg response with a dip of about $-5 \mathrm{~dB}$ at a wavelength of $1.54 \mu \mathrm{m}$. The deficiencies in this modeling technique were indicated above and are enhanced when the grating index is increased above the waveguide index. The perturbation theory spectrum (figure 4.3 middle) predicts a Bragg response that is about three times stronger than the FDTD computed response. The error can be traced to the fact that the perturbation technique assumes that the perturbation is small which is valid for a thin overlay thickness but invalidated when the overlay index is large compared to the waveguide layer index. For example, the theory assumes the perturbations to be small and in the case of the highdielectric overlay, the dimensional change in the waveguide cross-section is indeed small. 43 
However, the change in dielectric index is not small. Perturbation theory does not account for the variation in effective index which is a vital factor in determining the location of the Bragg dip and optimization of the grating's duty cycle. When the variation in effective index is taken into account, the two quarter-wavelength sections that make up a grating pitch are not the same physical length and this changes the solution to the Bragg condition. When this variation is not taken into account such as by the Fourier series square wave expansion of the perturbing grating, the results yield a practically unvarying Bragg dip location regardless of the index of the overlay and the corresponding effective index. The offset component of the expansion can be used in such a coupled-mode model to shift the dip wavelength according to the change in the refractive index of the overlay which changes the effective index. The perturbation method also assumes that the structures being modeled have modes well confined to the waveguide layer. As shown in figure 3.3 for this index increase, the mode's field amplitude maximum is shifted towards the waveguide-overlay interface resulting in significant field amplitudes in the overlay. The difference in the mode profiles in the 3-layer and 4-layer regions of the grating result in a coupling of power into the superstrate and substrate modes, not included in the perturbation analysis equations highlighted above. The 4-layer mode is shifted towards the overlay and has significant evanescent tail. It is possible to re-derive the perturbation model with the inclusion of the dropped terms in equation (4.4) and coupling to superstrate and substrate modes, these added complexities are avoided by selecting the highly accurate FDTD technique. 

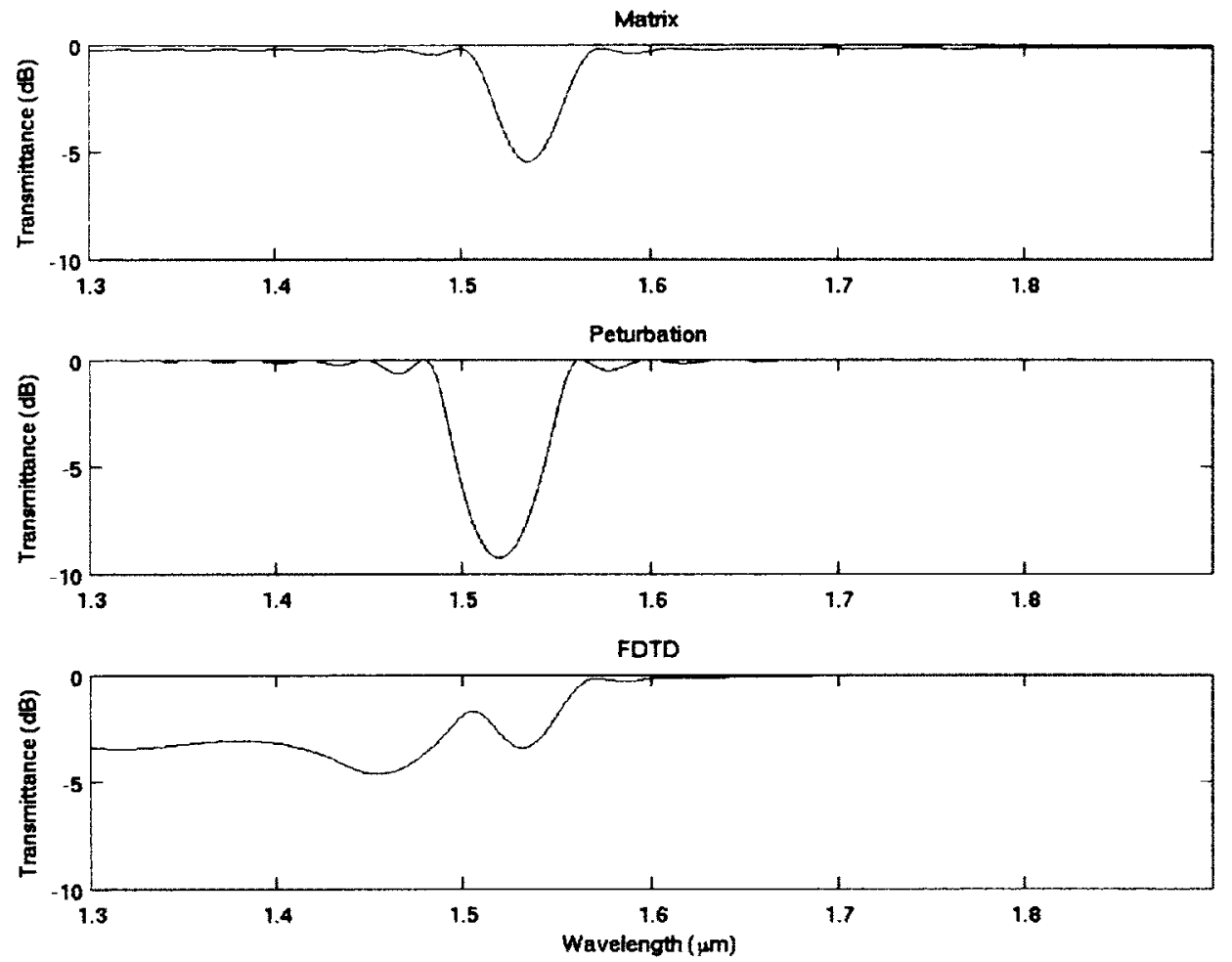

Figure 4.3 Shape of transmission dips for $\Delta n=1.5$ showing Bragg dip location variation for different modeling methods.

Figure 4.4 shows the shape of transmittance spectrum for $\Delta n=2.0$ providing an overlay refractive index of 3.6. The matrix approach estimates a Bragg dip greater than $20 \mathrm{~dB}$ while the perturbation approach estimate a dip less than $20 \mathrm{~dB}$. The Bragg dip obtained by FDTD is reduced demonstrating that coupling to the grating layer as a waveguide is occurring and that at the low wavelength range the coupling to radiating modes has increased. The two other modeling techniques can no longer be applied and the overlay grating becomes a sub-wavelength grating waveguide.[6] 

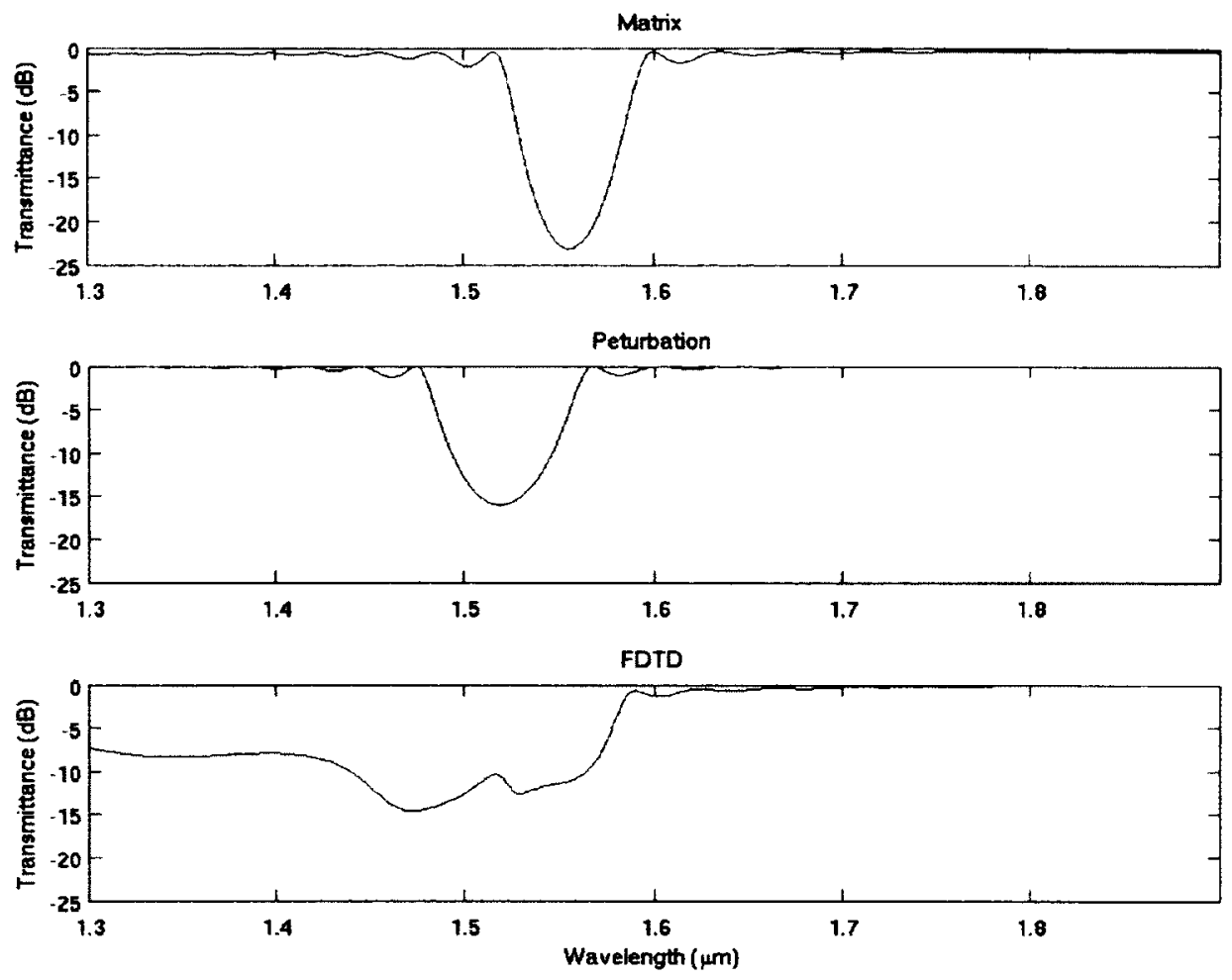

Figure 4.4 Shape of transmission dips for $\Delta n=2.0$.

An additional set of transmittance spectra is shown in figure 4.5 for $\Delta n=1.88$ representing the specific case of a silicon overlay layer of $n_{2}=3.48$. In the FDTD transmittance spectrum the presence and location of the Bragg dip is well defined since the overlay layer is not acting as a guiding layer. The secondary dip shown in the spectrum blocks out low wavelengths while wavelengths above the primary Bragg dip experience full transmission. Considering these properties in terms of wavelength, the waveguide with a thin grating patterned overlay is equivalent to a high wavelength or low frequency pass filter.[9]. 

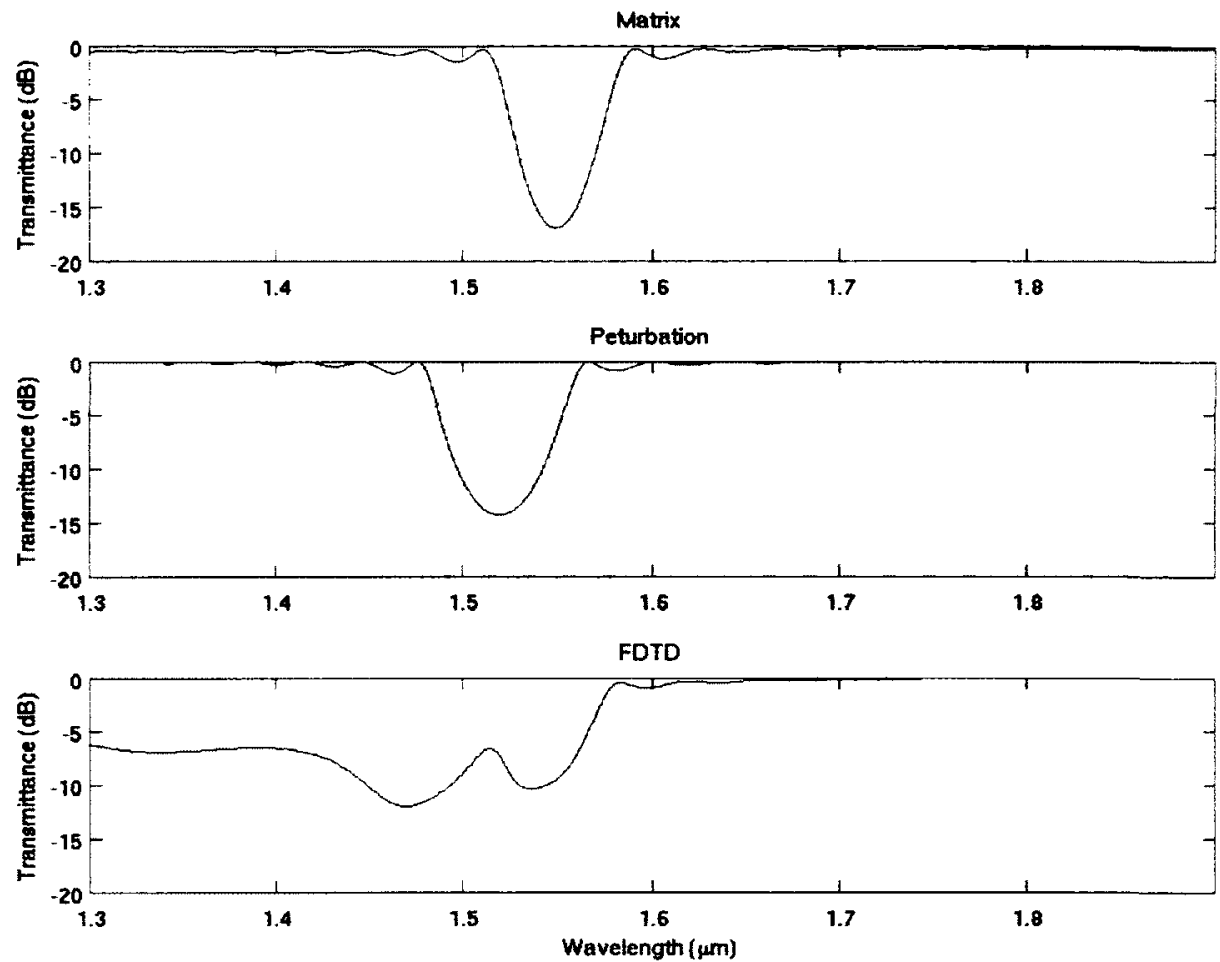

Figure 4.5 Shape of transmission dips for $\Delta n=1.88$ which represents the use of a silicon overlay on a silica waveguide.

Figure 4.6 plots the transmission minimum of the Bragg dip (in $\mathrm{dB}$ ) as a function of $\Delta \mathrm{n}$ from 0.0 to 2.0 . The FDTD curve is considered accurate as determined through convergence tests. As can be seen, the simpler modeling techniques overestimate the strength of the Bragg dip. Plotted in figure 4.7 is the difference in the minimum of the Bragg dips referenced to that of the FDTD technique. Setting a minimum allowable difference of $1 \mathrm{~dB}$ indicates that the matrix approach can be used up to a $\Delta \mathrm{n}$ of 1.17 and the perturbation approach can be used up to a $\Delta \mathrm{n}$ of 0.53 . Beyond these values, the simpler techniques should not be used. 


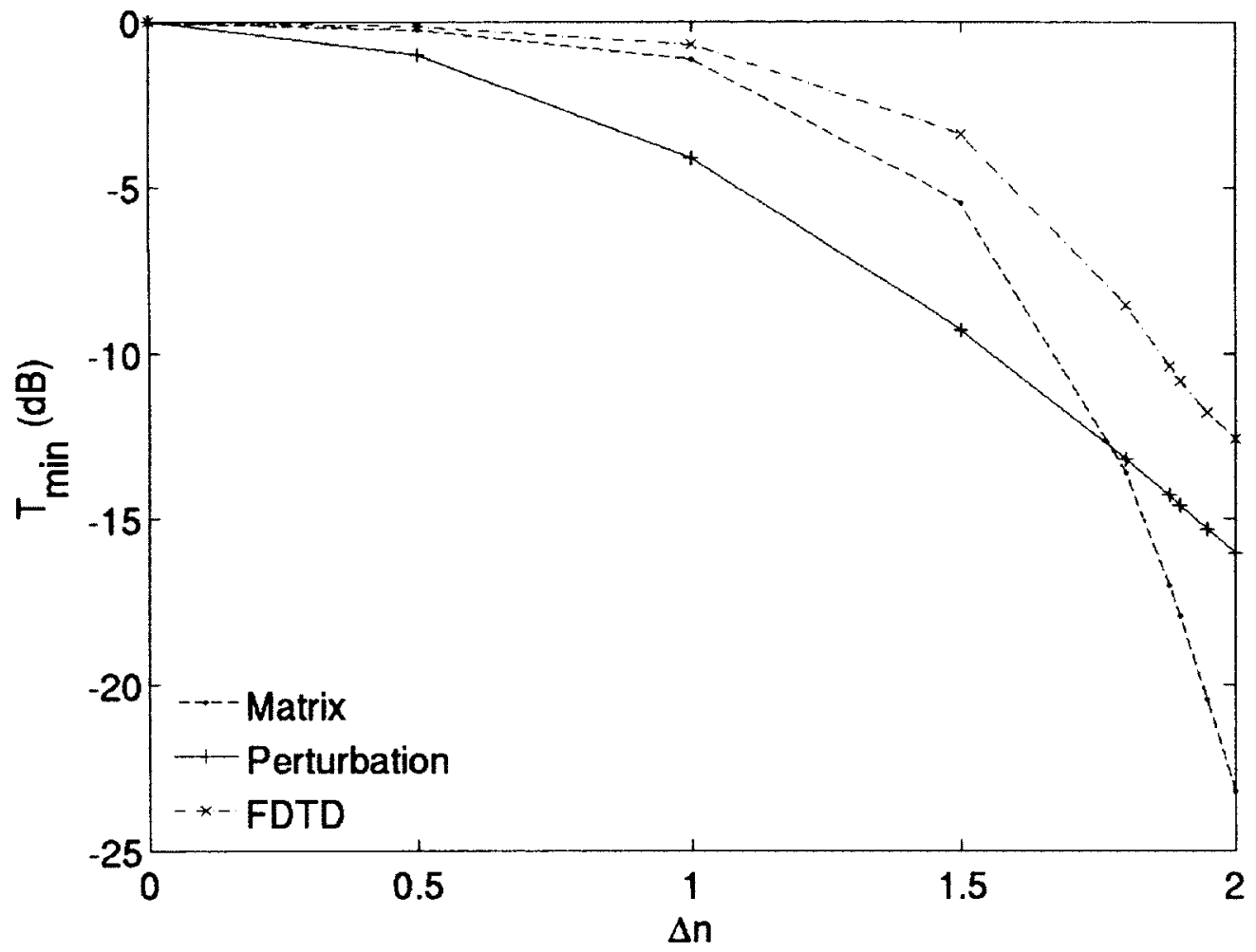

Figure 4.6 Depth of transmission dip versus An showing that the matrix and perturbation techniques overestimate the strength of the Bragg dip with respect to FDTD. 


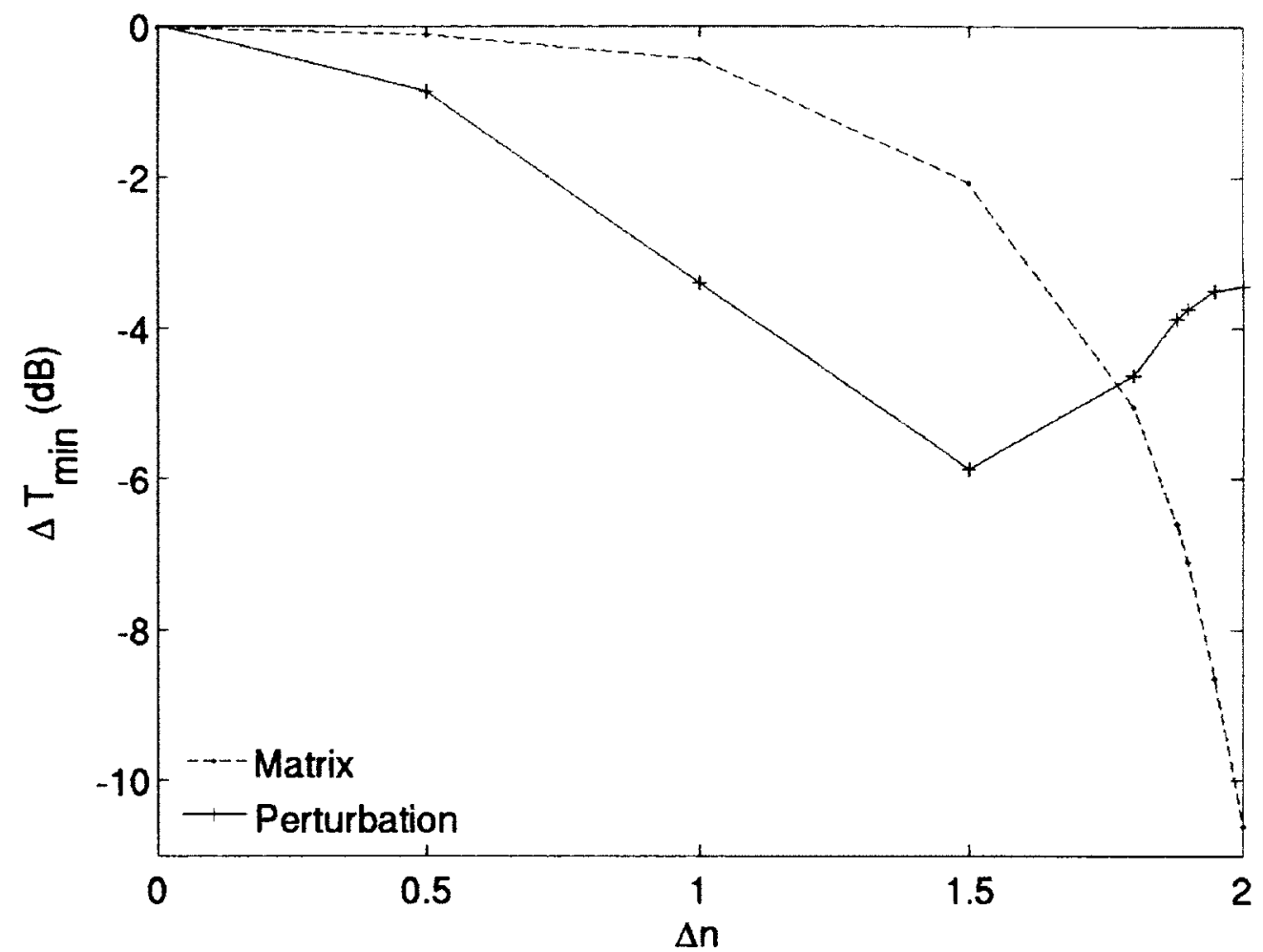

Figure 4.7 Difference in the depth of transmission dip with respect to FDTD versus $\Delta$ n showing that the matrix method is within $1 \mathrm{~dB}$ until $\Delta$ n exceeds 1.17 .

Figure 4.8 plots the Bragg dip wavelength versus $\Delta \mathrm{n}$ from 0.0 to 2.0 . The matrix technique returns consistently higher wavelength values when compared to the FDTD result while the perturbation approach returns consistently lower values. Both the matrix and perturbation approaches exhibit slight changes in their trends within or slightly above the design range. The FDTD curve begins with a trend towards higher wavelengths, then exhibits the onset of the sub-wavelength grating effect near the end of the design range when the Bragg dip trend reverses. This phenomenon occurs between the faint line indicating when the peak of the mode enters the overlay and the faint line indicating when the effective index of the guided mode in the four-layer sections exceeds the 49 
guiding layer refractive index. As per chapter 3 , the field solution in the guiding layer changes from sinusoidal to exponential when the effective index exceeds the guiding layer refractive index. In this transition region the Bragg dip depth does not continue increasing at the previous rate and the dip is also broader due to the transmission through the sub-wavelength grating, which behaves as an index guide. When the index of the overlay continues to increase, the overlay becomes a sub-wavelength grated waveguide akin to periodically segmented wave mode converters [5] or sub-wavelength periodic structures [6]. Note that there is a plateau at the maxima of the wavelength shift shown by the FDTD method at the refractive index of silicon. This plateau represents a range of overlay refractive indices for which the structure provides a similar response permitting material parameter tolerance. This tolerance allows for fabrication error, for carrier injection, and for doping including for the creation of junctions. Figure 4.9 shows a plot of the difference in Bragg dip location referenced to that of the FDTD value as a function of $\Delta \mathrm{n}$. Selecting a maximum acceptable Bragg dip wavelength error of $2 \mathrm{~nm}$ indicates that the matrix approach may be used up to a $\Delta n$ of 0.83 and the perturbation approach can be used up to a $\Delta \mathrm{n}$ of 0.56 . Combining these restrictions with those of the Bragg dip strength indicates that maximum range for each technique to provide accurate results. 


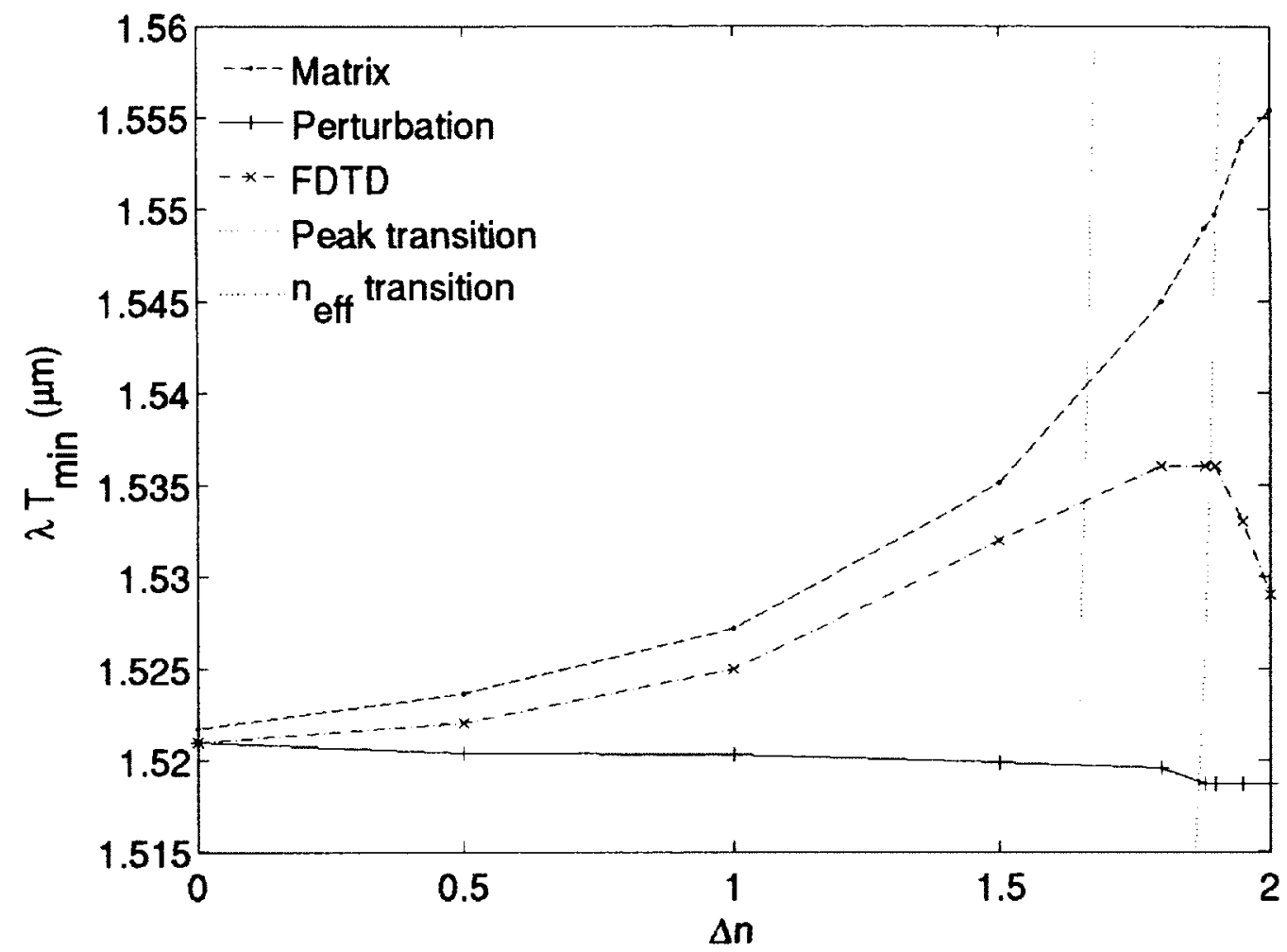

Figure 4.8 Transmission dip wavelength versus increasing $\Delta n$ showing the matrix method overestimates and the perturbation method underestimates the wavelength with respect to FDTD.

The first faint line denotes increase of modal effective index such that the peak enters the overlay near the desired point of operation while the second faint line indicates when the effective index exceeds that of the guiding layer's material refractive index . 


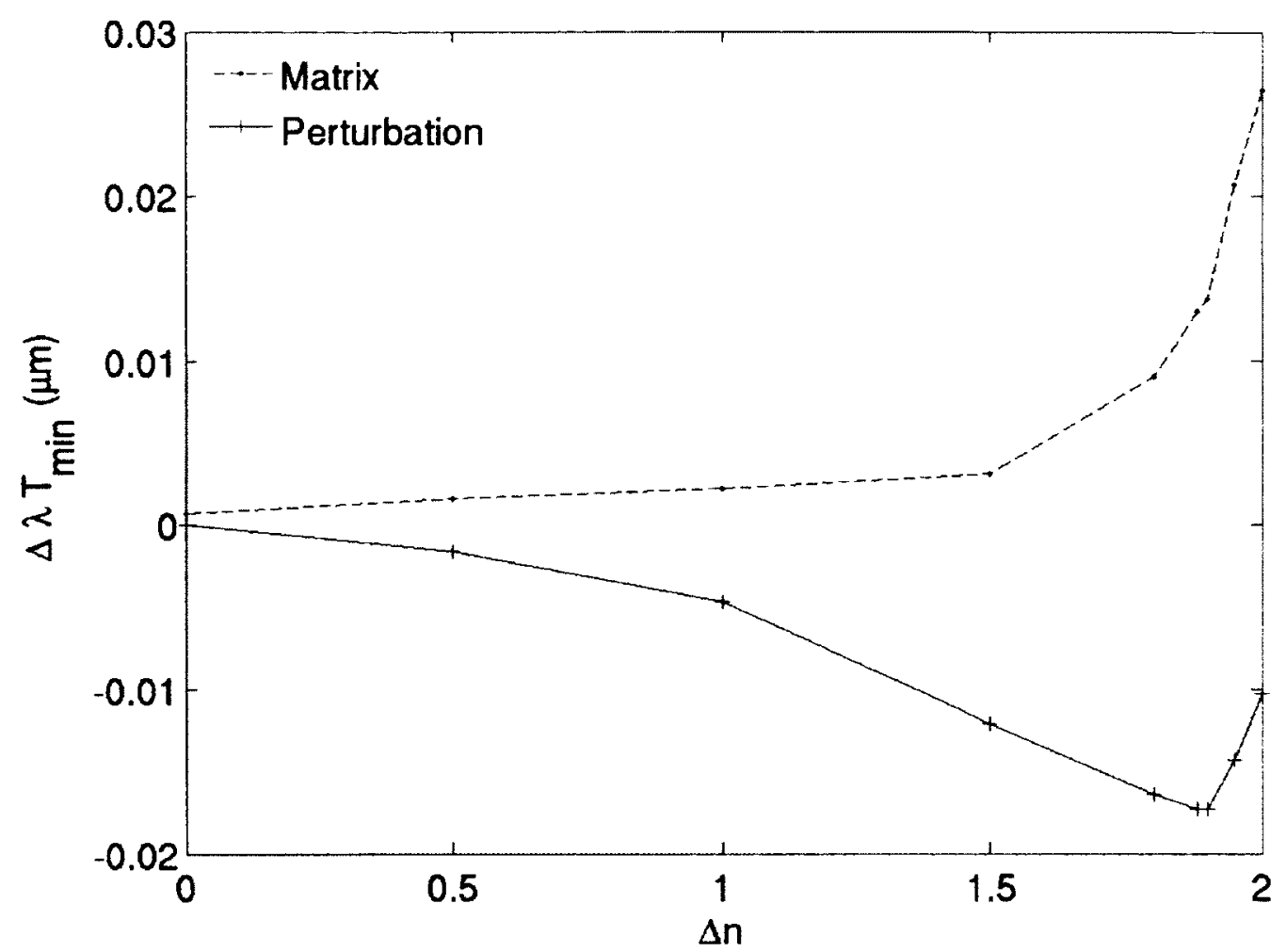

Figure 4.9 Difference in the transmission dip wavelength with respect to FDTD versus $\Delta \mathrm{n}$.

\subsection{Modeling findings}

The methodology for identifying the behavior of a ID photonic crystal overlay on glass has been examined using a matrix-based technique, a perturbation approach, and FDTD simulation. The first two approaches were compared to the FDTD simulations. The matrix approach is useful for initial design of an overlay structure. It provides reasonably accurate predictions of the strength and location of the Bragg dip until the refractive index of the overlay approaches and exceeds that of silicon. At that point, the matrix method over estimates both the Bragg dip strength and shift with increasing overlay refractive index. Perturbation theory is valid for small $\Delta \mathrm{n}$ which if erroneously extended to larger $\Delta \mathrm{n}$ predicts an incorrect strength and a practically unvarying location for the 52 
Bragg dip. However, it predicts a reasonable value for the strength of the Bragg dip for overlays with refractive index approaching and exceeding that of silicon. This allows for the design of high dielectric contrast overlays in cases where the matrix method overestimates the magnitude of the structure's effect. Modifying either method to better represent the structure increases their complexity thus tuning of a design requires FDTD, a more comprehensive approach that is straightforward to apply. It is used in chapter 5 and chapter 6 to further investigate high refractive index dielectric overlay structures. Using FDTD has also demonstrated that there is a range of refractive index for this structure in which the location of the Bragg dip in wavelength does not change appreciably for changes in overlay refractive index allowing for material tolerance which can be for fabrication error, for carrier injection, and for doping. 


\section{FDTD 2-D simulation}

As shown in the previous chapter, the Finite-Difference Time-Domain (FDTD) method is the preferred approach for this work. The FDTD approach as described in textbooks does not make the assumptions made for the other techniques and thus continues to provide correct results, when tested for convergence, when those assumptions are no longer valid for the other approaches. The FDTD method[68,69] can be used for a variety of electromagnetic simulations and has been implemented in numerical software code[30]. With respect to this work, FDTD simulations are used to determine reflection and transmission spectra of photonic structures as well as to investigate their other optical properties. Tests have shown that the simulations can take a long time to complete and both optimization and time duration issues are addressed in Appendix B. This chapter provides additional insight into FDTD simulations of the structures described in section 4.4. The next chapter describes further simulations of novel structures.

\subsection{Application of FDTD to the patterned overlay system}

In the previous chapter, section 4.2 addressed the patterned overlay system and it was modeled using FDTD simulation in section 4.4. The specific parameters as to how the technique was applied were described and the results for increasing overlay refractive index dielectric contrast were presented. Here we will continue to further investigate the characteristics of the FDTD simulation of the patterned overlay system. Figure 5.1 presents the waveguide structure featured in Figure 4.1 discretized on a square grid at 160 points per micron including the additions described in section 4.4. The source described in (4.27) has a spectral extent that covers the wavelengths of interest by centering it at a wavelength of $1.55 \mu \mathrm{m}$ with a width $w=0.3340$. The pulse is visible in Figure 5.2 after 54 
the simulation has run for 10,000 time steps in the 3 layer structure (top) and 4 layer structure (bottom). The figure shows that in the 3 layer case the pulse is guided and all frequency components travel in the propagation direction. In the 4 layer case a well

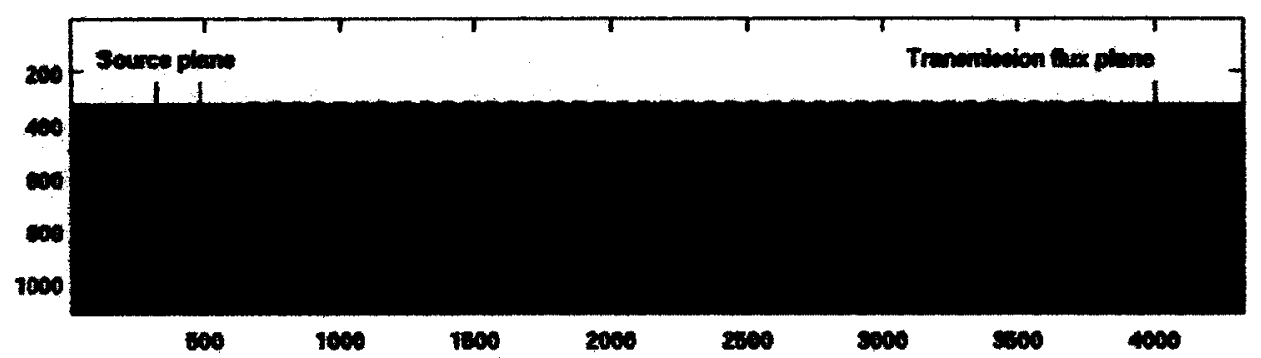

Figure 5.1 Standard simulation configuration with source and flux planes indicated. The numerical values on the axis denote the discretized FDTD grid coordinate values of the structure.

guided portion of the pulse remains while the back reflected frequencies including

\section{1}

\section{1}

Figure 5.2 Simulation after 10,000 time steps showing source pulse propagation in 3 layer structure (top) and 4 layer structure (bottom). The three layer structure allows the pulse to propagate unchanged while the four layer structure couples specific wavelengths to backward travelling and substrate modes.

55 
coupling to the guided backwards traveling mode can be seen. Radiation of energy into the substrate can also be observed which is further investigated by considering the energy that flows through neither the transmission flux plane nor the reflection flux plane. In Figure 5.3 the spectrum of the source pulse is show next to the spectrum of the pulse after it has propagated through the three layer waveguide structure further demonstrating the guidance of the pulse. Spectrum simulations were run for 60,000 time steps in order for the source pulse to propagate through and reflect off the structure, possibly resonate and decay, as well as be absorbed by the PML.
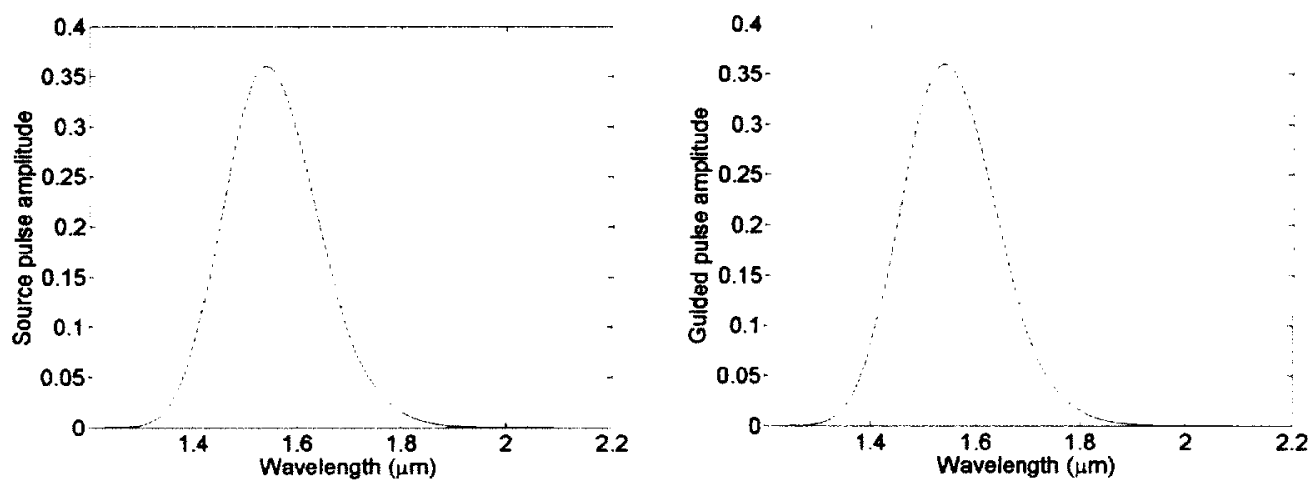

Figure 5.3 Spectrum of the source pulse (left) next to the spectrum of the propagated pulse (right) showing minimal change of the source pulse when it propagates along the 3 layer structure.

Figure 5.4 shows the flux through the reflection plane on the left and the transmission plane on the right respectively for the full structure which includes the thin periodic patterned silicon overlay. As shown to scale in Figure 5.1, these flux planes are located 1 $\mu \mathrm{m}$ from their respective ends of the grating. Subtracting the reflection plane flux from the incident pulse provides the backward travelling flux that has been reflected by the structure. This flux can be normalized by dividing by the incident flux measured in the un-perturbed structure providing the normalized reflectance spectrum. Similarly the 56 
transmitted flux can be normalized by using the transmitted flux measured in the unperturbed structure providing the transmittance spectrum. These spectra are shown in Figure 5.5.
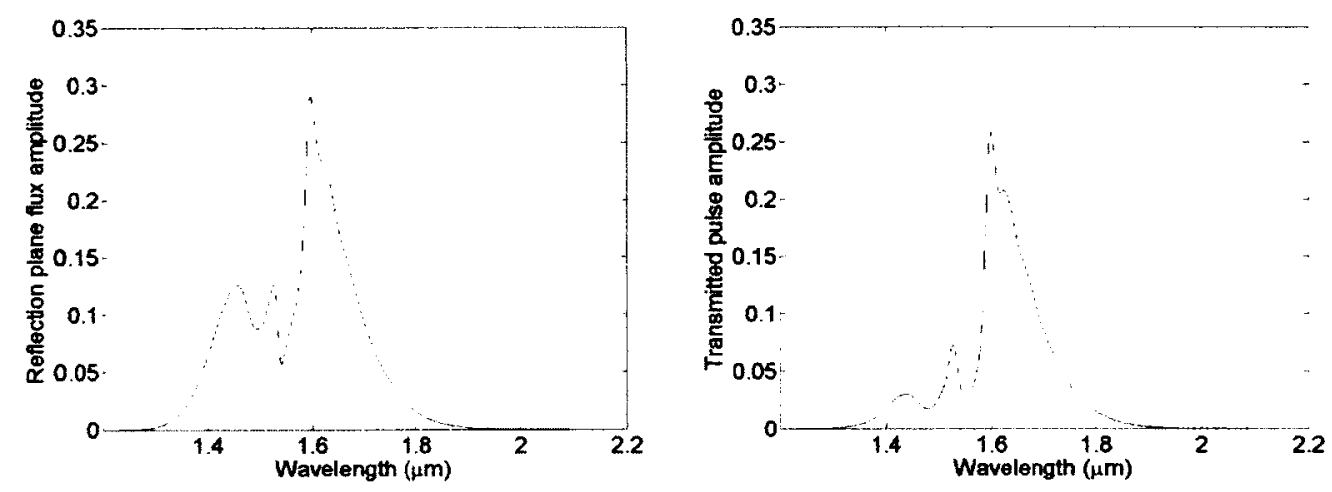

Figure 5.4 Spectrum of the reflection plane flux (left) prior to separating forward and backward travelling components next to the spectrum of the transmission plane flux (right). The reflection flux plane measurement contains both the source flux and the reflected flux while the transmission flux plane measurement contains the transmitted portion of the source flux.
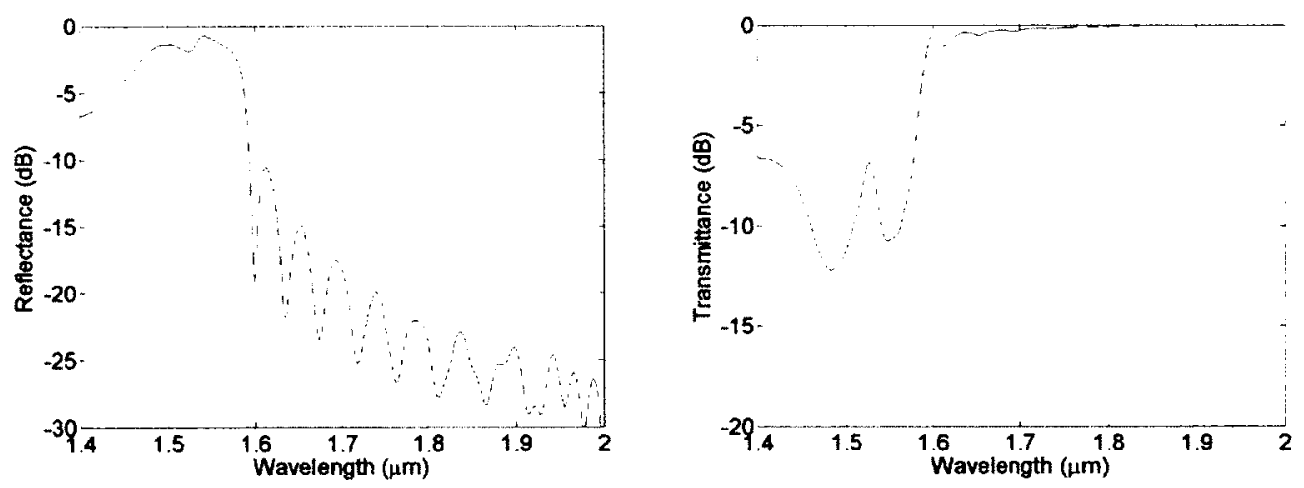

Figure 5.5 Reflectance (left) and transmittance (right) spectra.

Additional flux planes are considered to distinguish between the flux in and near the overlay from that of predominantly the guiding layer. The first set follows the existing flux planes $100 \mathrm{~nm}$ into the waveguide layer and the second continues from that 57 
breakpoint onwards as per the existing flux planes. Thus the set of reflectance and transmittance spectra for both the overlay and guiding layer can be obtained as shown in Figures 5.6 and 5.7 respectively. The amount of energy travelling in the portion of the structure where the periodic overlay appears is significantly smaller and thus note the change in vertical axis scale in Figure 5.6 made in order to better observe the traces.
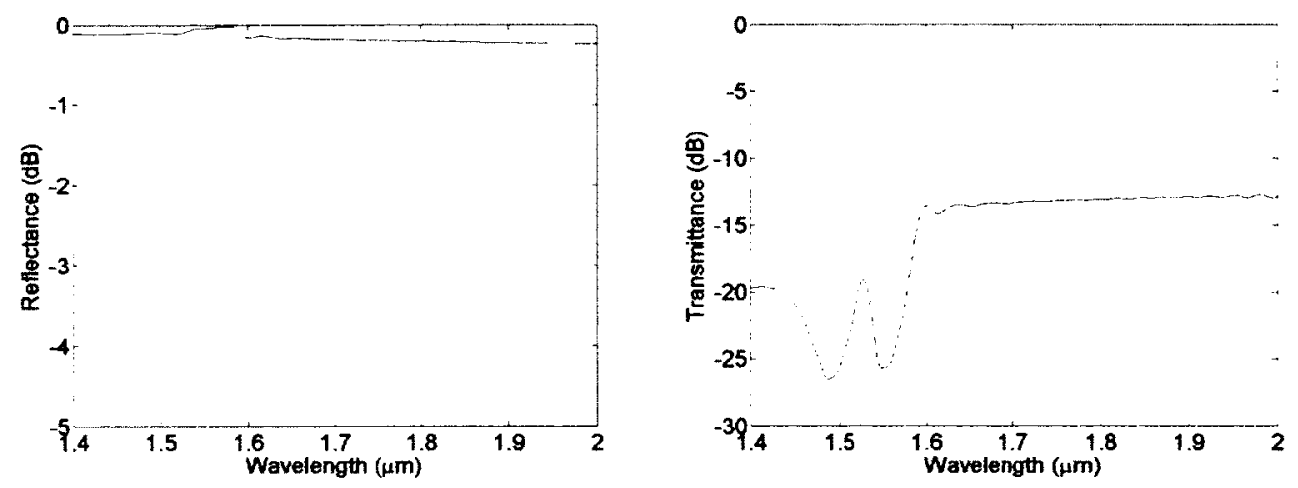

Figure 5.6 Reflectance (left) and transmittance (right) spectra representing light travel predominantly in line with the overlay.

It is apparent that, as shown in Figure 5.7, the majority of the energy travels in the guiding layer.
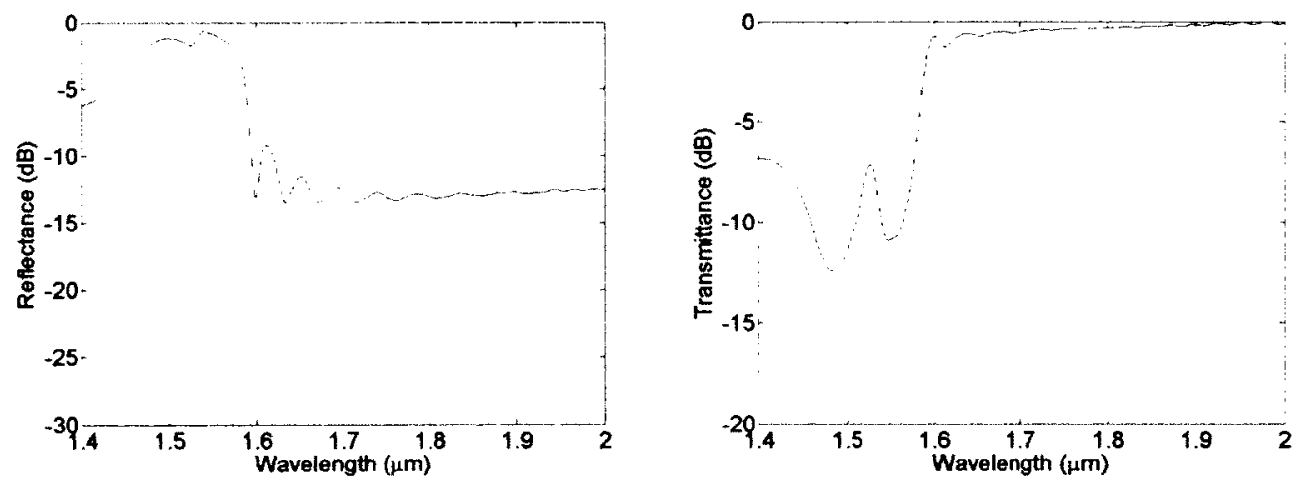

Figure 5.7 Reflectance (left) and transmittance (right) spectra representing light travel predominantly in line with the guiding layer.

58 
The larger the simulation space is, the longer the simulation time. Thus, in order to ensure that the simulation space has not been reduced at the expense of accuracy, results are compared when the PMLs are moved further from the periodic overlay. In Figure 5.8 the results are presented for when $10 \mu \mathrm{m}$ three layer waveguide sections are added in the propagation direction both before and after the periodic overlay section. This simulation was run for 94,000 time steps.
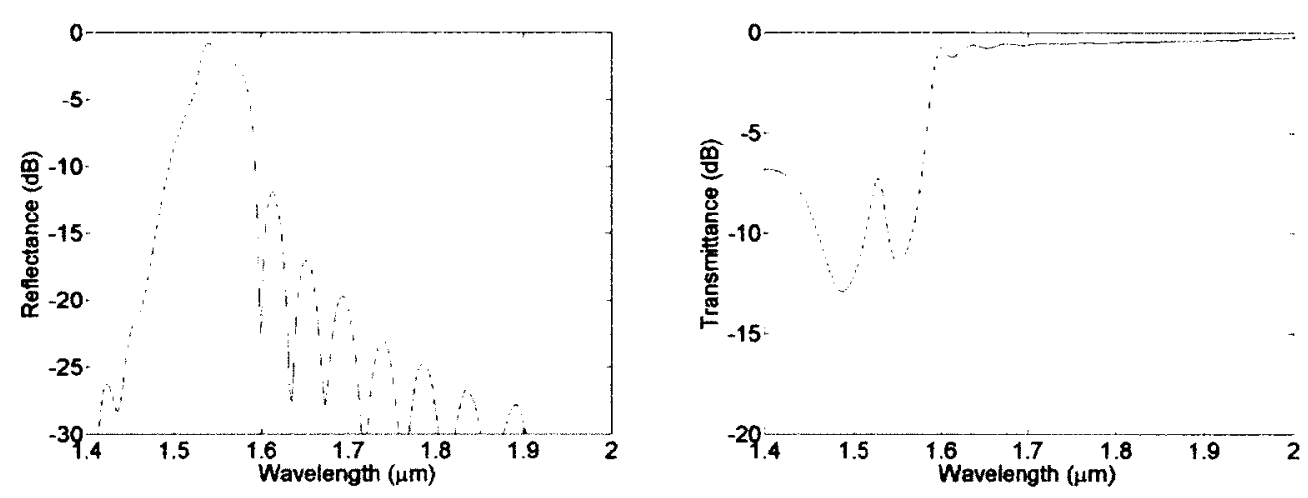

Figure 5.8 Reflectance (left) and transmittance (right) spectra for structure with $10 \mu \mathrm{m}$ lead in and lead out.

The source and the flux planes are moved an additional $90 \mu \mathrm{m}$ further away for the spectra shown in Figure 5.9 to verify accurate representation. This simulation was run for 454,000 time steps and took about 10 days to complete. These simulations have shown that matching the source profile to the three layer field solution of the central wavelength of the pulse allows the smaller simulation domain without requiring a propagation section for its evolution. The reflection plane does pick up a bit more flux on the low wavelength side when it is close to the periodic overlay demonstrating that it is picking up scattered light that would otherwise be absorbed by the PML as shown in Figure 5.10. 

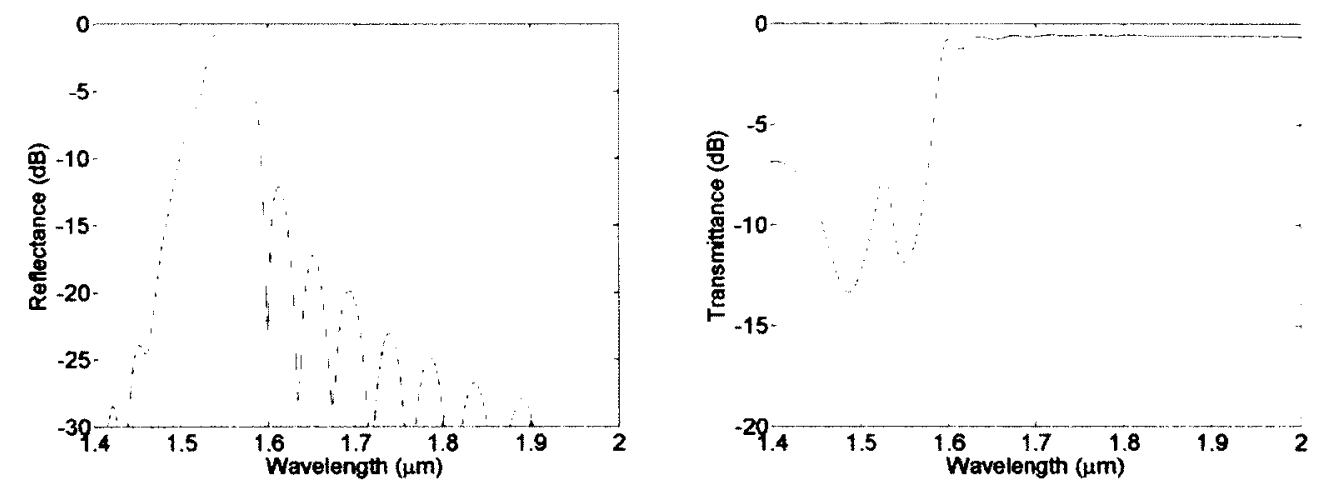

Figure 5.9 Reflectance (left) and transmittance (right) spectra for structure with $100 \mu \mathrm{m}$ lead in and lead out.
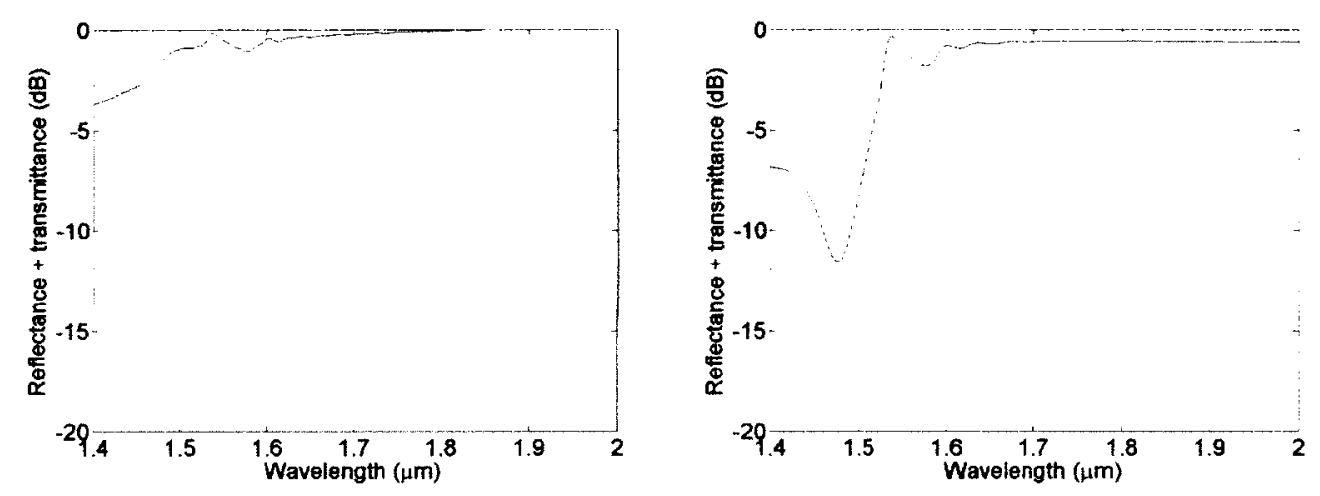

Figure 5.10 Spectra of the sum of reflectance and transmittance spectra for compact simulation domain (left) and for structure with $100 \mu \mathrm{m}$ lead in and lead out (right).

In order to be able to more accurately examine the structure's response for specific wavelengths a single frequency source was used in the simulation and the field distribution was observed. Leaving the source on continuously and waiting for the steady state makes it difficult to distinguish standing waves from travelling waves in a view of the field distribution for a specific simulation time step. One solution to this is to retain the field distributions for all simulation time steps, convert them into images that are then used as frames of a movie. Storage space required for the raw field data is tremendous. 60 
Storing only the images relieves but does not eliminate the storage space requirements. It was found that not every frame was required to be able to view a smoothly progressing field in the movie files. Dropping frames further reduces the requirements for both the storage of the movie itself and the computation involved in creating it. When the field distributions and respective images for the unneeded frames are neither created nor stored this improves simulation efficiency. Another method for distinguishing flow of light in the images was to use a pulse of the single frequency source. In this case when energy is found near the source when it is off, it is known that it is has been reflected by the structure. Another benefit of this strategy is that it is not necessary to continue to iterate until a steady state point. Gradual turning on and off of the source amplitude reduces the artifacts from the step-like turn on and off of the source but adding this increases fading and make it more difficult to distinguish exactly what was occurring with the light waves. Thus, field images were generated and stored for key points but when simulation efficiency was required for a multi-frequency response spectrum none were taken. In a similar manner to multi-frequency pulse shown in Figure 5.2, Figures $5.11,5.12$, and 5.13 show the single frequency pulses at 10,000 time steps. In Figure 5.11 the field is shown for the pass-through wavelength of $1.800 \mu \mathrm{m}$ and it is clear that the source pulse is travelling in the guide without the periodic overlay having a significant effect. In Figure 5.12 the field is shown for the design wavelength of $1.550 \mu \mathrm{m}$ where it is clear that the source pulse has been reflected back towards the source along the guide along with some scattering. While in Figure 5.13 the field is shown for the secondary dip wavelength of $1.500 \mu \mathrm{m}$ where the energy is also reflected and scattered at a bit more of an angle to the 
guide clarifying the findings of Figure 5.10 where, given more space, the unguided energy is found to be absorbed by the PML.

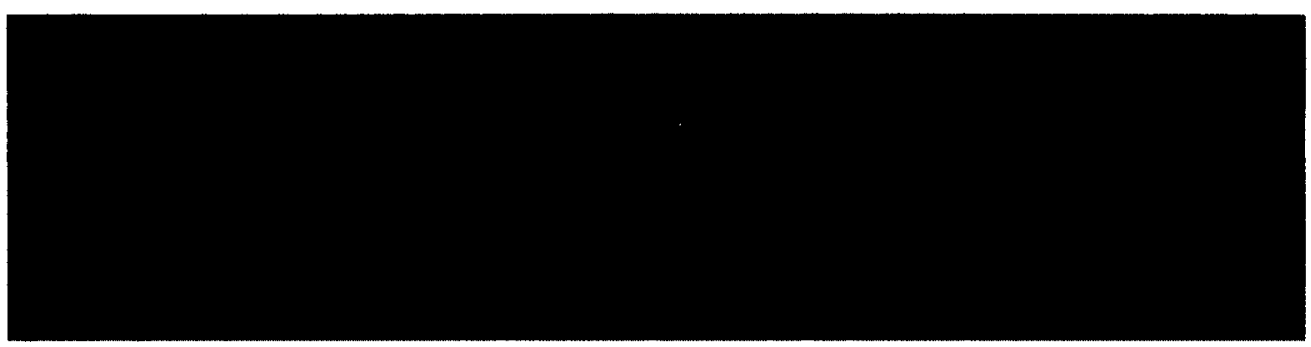

Figure 5.11 Simulation after 10,000 time steps showing $1.800 \mu \mathrm{m}$ pulse propagation.

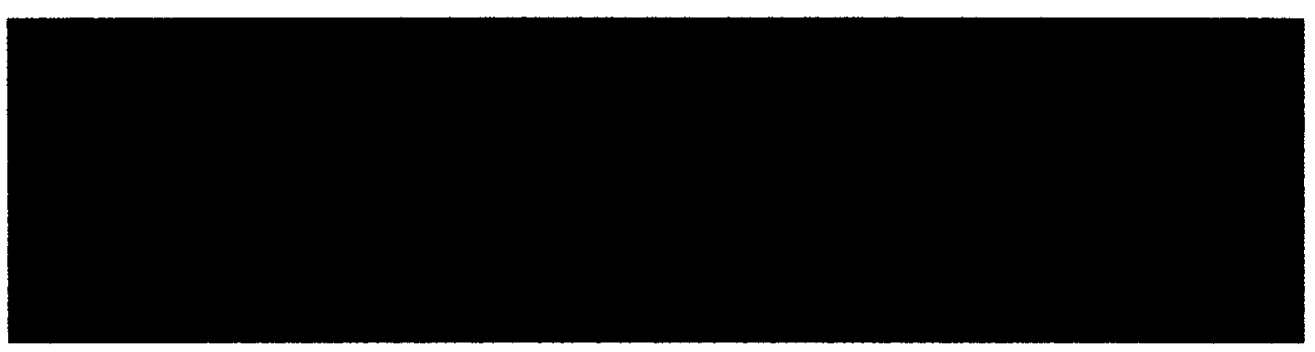

Figure 5.12 Simulation after 10,000 time steps showing $1.550 \mu \mathrm{m}$ pulse propagation.

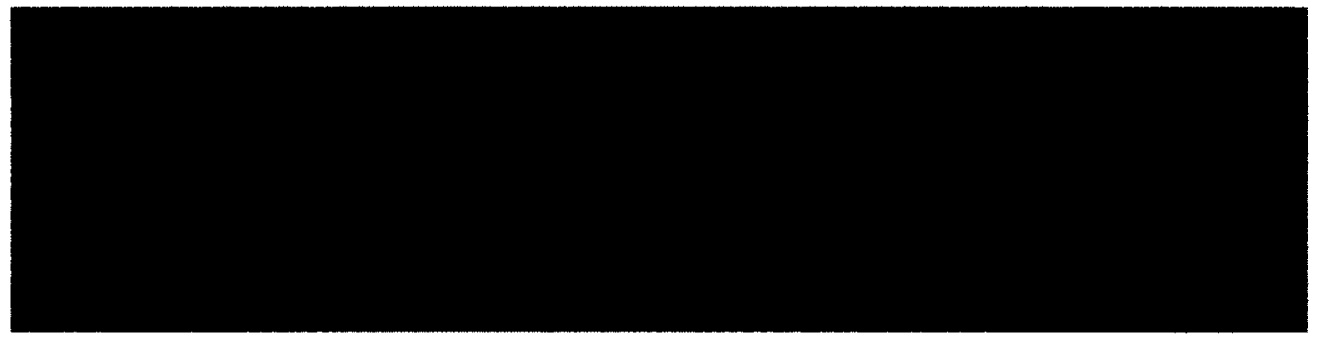

Figure 5.13 Simulation after 10,000 time steps showing $1.500 \mu \mathrm{m}$ pulse propagation.

In the next chapter, the structure is further examined, modified, and refined for specific novel applications. As previously mentioned in section 4.4 , the materials used are considered to be without material dispersion. However, in the sensor application, a material[77] is used that has more material dispersion. Thus before proceeding, it is pertinent to consider material dispersion in that case to ascertain how the added 
complexity of including it compares to results obtained assuming no material dispersion.

Figure 5.14 demonstrates the effect of material dispersion on sensor response by comparing the solid trace of a typical simulation with the dashed trace of the more complicated simulation including material dispersion by varying the refractive index with wavelength. The traces are superimposed throughout the central range of wavelength of interest while at the extremities the tendency is for the spectral response including material dispersion to be stretched slightly with regard to wavelength resulting in a slight reduction in slope. The effect is negligible, especially when considering that the sensor device operation centers around a wavelength of $1.55 \mu \mathrm{m}$ and that transmission is switched off at a wavelength of $1.57 \mu \mathrm{m}$.

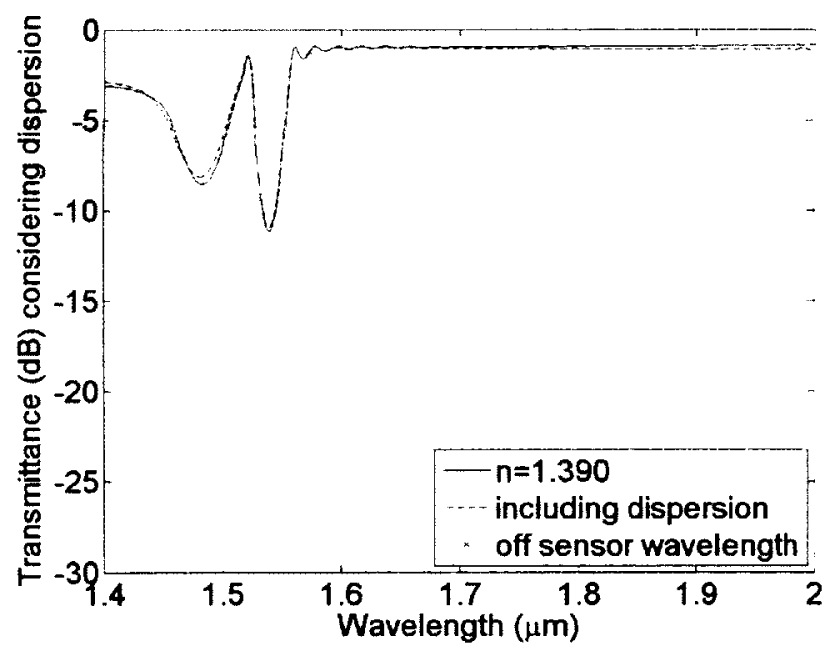

Figure 5.14 Simulation including material dispersion to show effect on sensor response at $1.57 \mu \mathrm{m}$.

The basics of the FDTD method have been described in the first section of this chapter. Subsequent sections outline the considerations involved, including limitations to be aware of and operated within, such as ensuring convergence, with the use of the FDTD method for the specific structure described by this work. Additional simulations 63 
explored in this body of work are included in Appendix C, Catalogue of simulations. The use of the described FDTD simulation domain is understood to represent the transmission spectrum in an accurate manner and is used for the published results described in the following chapter. 


\section{FDTD of Novel 2-D layouts}

This chapter presents the FDTD simulation of the novel 2-D layout. The work completed to design this layout can be expanded to utilize the platform for more complex photonic crystal designs. The design and objective of the structure is followed by the structural design details which are used to model that structure for FDTD simulation. The results of the simulations for the structure are followed by a comparison and performance evaluation.

In chapter 3 the constraints to maintain single mode operation in overlay structures were explored. In chapter 4 the use of modeling methods including FDTD for these structures was discussed and in the previous chapter the FDTD simulations of these structures were addressed. The structure described by this work operating in the single mode regime is analyzed in a parametric analysis followed by an application as a sensor.

\subsection{Patterned overlays: thin silicon layer applied to glass waveguides}

This section covers the parametric analysis of patterned overlays as presented in published work[9]. A three layer glass waveguide is modified into a 4 layer structure by adding a thin high dielectric overlay at the air-waveguide interface. The next section presents the details of the waveguide geometry and modal parameters obtained for uniform overlays. Considerations related to the patterning of the overlay follow. Next, a study of the optical transmission and reflection of the waveguide geometries is provided for the condition when the overlay parameters are set to typical values. A brief discussion of an optical filter designed from a thin patterned high dielectric clad glass waveguide completes the section. All un-patterned overlay waveguide configurations are analyzed 65 
by solving Maxwell's equations for the 3 and 4 layer structures while patterned overlay waveguide configurations are analyzed using the FDTD computation technique.

\subsubsection{Design and objective}

The basic 4 layer slab waveguide structure is shown in figure 6.1 (left). The substrate index is taken as glass, $\mathrm{n}_{4}=1.500$ and extends to infinity away from the glass waveguide layer of thickness, $t_{3}=1 \mu \mathrm{m}$, and index, $\mathrm{n}_{3}=1.600$. The overlay has a thickness, $\mathrm{t}_{2}$, and index, $\mathrm{n}_{2}=3.480$ and is assumed uniform over the waveguide. The superstrate extends to infinity above the guide and has index, $n_{1}=1.000$. The individual layers lie in the $(y, z)$ plane and the light's propagation direction is chosen along $y$. The index of refraction profile is shown in figure 6.1 (right) and demonstrates the high dielectric contrast available between glass waveguide layer and the overlay. The thickness and index of refraction values chosen for the waveguide are compatible with fibre based waveguide dimensions when the slab configuration is restricted in the $\mathrm{z}$ dimension making it a channel in nature. Given the index of refraction and layer thickness for the slab waveguide, the generic solution to Maxwell's equation indicates that modes of propagation may be possible with the maximum of the field component located in the high dielectric thin overlay layer. Such solutions would be periodic in this layer and exponentially decaying in the outer layers. Such modes of propagation are highly desirable for waveguide configurations built in the SOI platform, but, are not the types of modes of interest here. In the design of interest, the dielectric above and below the thin high dielectric layer are different and results in an asymmetric waveguide configuration

with respect to the thin high dielectric slab overlay. A convenient property of the asymmetric waveguide is the fact that it can demonstrate a lower mode cut-off for 66 

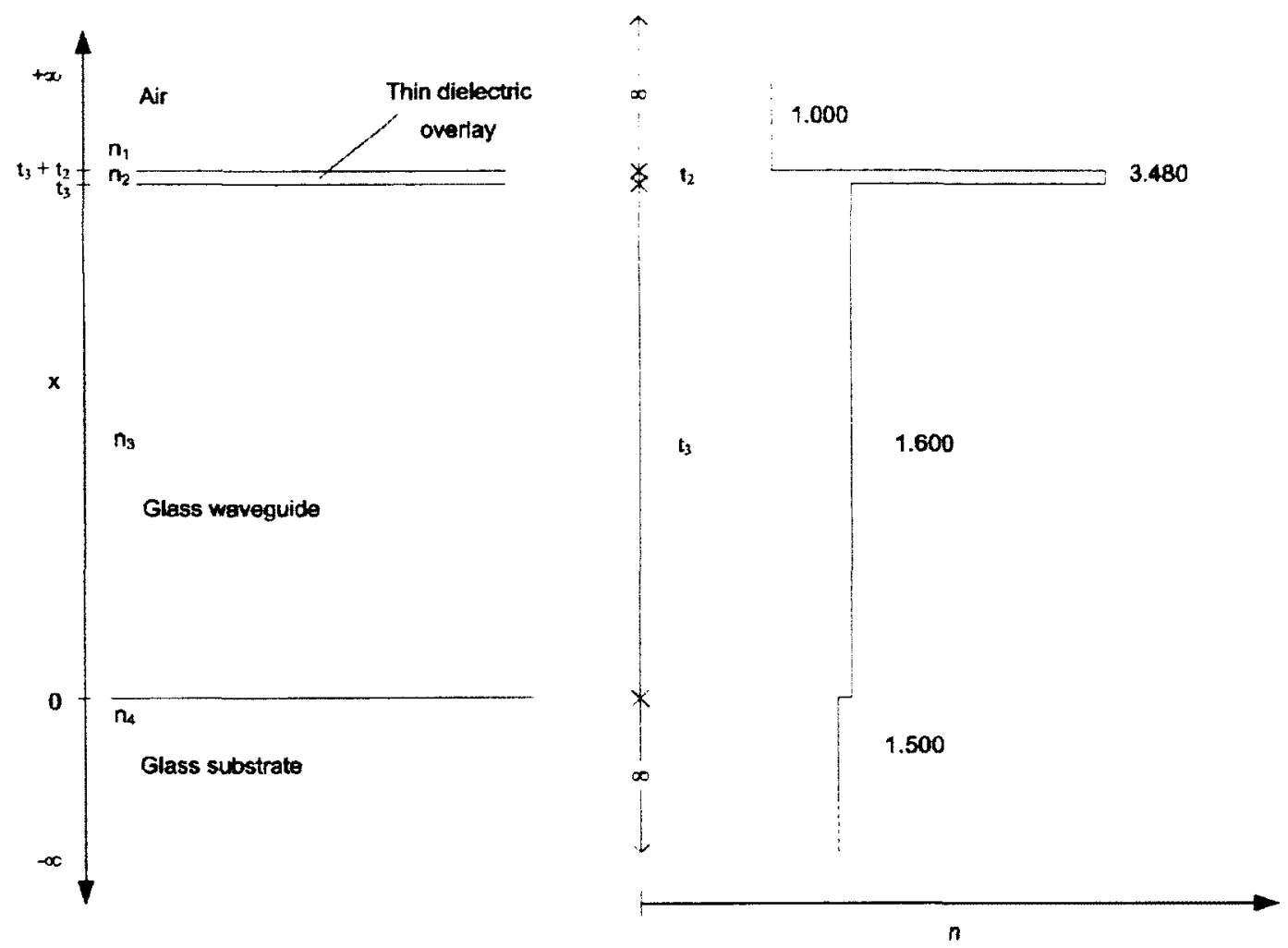

Figure 6.1 Left: four layer structure with $t_{3}$ and $t_{2}$ as the thickness of the thin film waveguide and overlay respectively. Right: corresponding refractive index profile.

which no effective mode indices lie above $n_{3}$. The lowest order mode of the entire structure can be designed to have an effective index between $n_{4}<\beta / k \leq n_{3}$ giving a peak in the mode profile near or in the glass waveguide layer. Thickness and index of refraction combinations are possible which result in a single mode waveguide with mode peak in the glass waveguide layer. The overlay has the effect of slightly pulling the mode up towards the higher dielectric region resulting in a significant field component present at the waveguide-silicon interface and in the thin overlay layer. Such field enhancement makes the mode sensitive to the properties of the overlay. The determination of the thicknesses for the 4 layer structure is obtained by solving the 4 layer slab waveguide 67 
configuration and ensuring that the lowest order mode has an effective index falling between $n_{4}<\beta / k \leq n_{3}$. In addition, the modal solutions are examined as a function of wavelength to ensure that the waveguide remains single mode throughout the communication wavelength band. The high dielectric layer is chosen to be $0.0347 \mu \mathrm{m}$ thick, giving a single mode to multi-mode waveguide transition below a wavelength of $1.414 \mu \mathrm{m}$.

Figure 6.2(top) shows the modal profile obtained for the single mode waveguide for a wavelength of $1.55 \mu \mathrm{m}$ when 4 layers are present. The modal amplitude is designed to peak in the region near the high dielectric overlay interface. This provides the largest field amplitude in the high refractive index dielectric thin layer, without having the mode take on an effective index in the high refractive index dielectric range. The equivalent mode solution when the high dielectric layer is removed in shown in the lower image of figure 6.2. All waveguide parameters are unchanged relative to the top image and the solution to Maxwell's equations indicates that this structure is also single mode. In both profiles, the peak of the mode profile is asymmetric due to the different indices of the superstrate and substrate. In a practical device configuration a transition from a 3 layer waveguide to a high dielectric clad overlay 4 layer configuration may exist. Mode overlap between the modes of the transition regions is calculated to be 0.77 . The coupling efficiency can be increased by smoothing the transition at the junction of the 3 and 4 layer waveguides. 

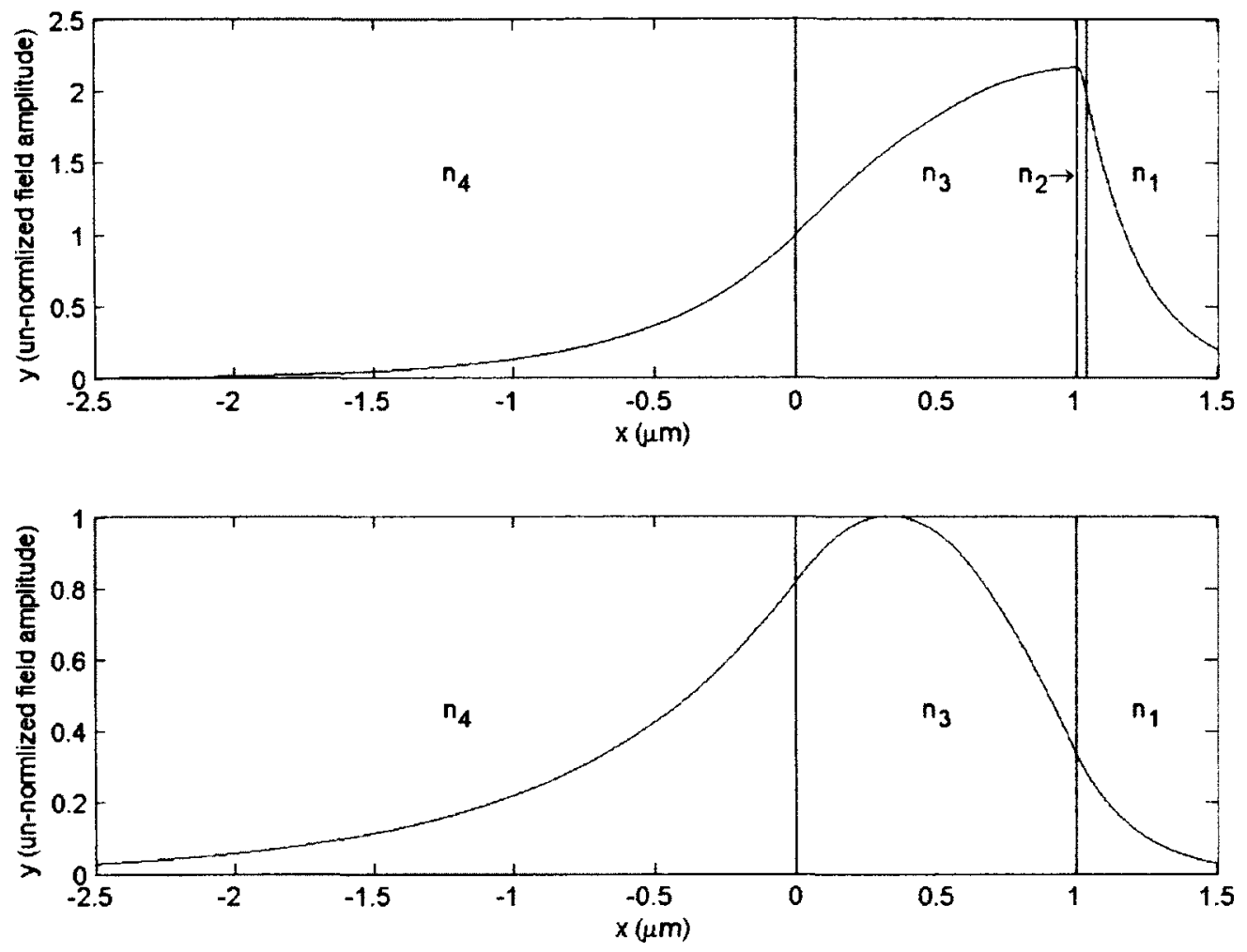

Figure 6.2 Four and three layer structure TE $\left(E_{Z}\right)$ field profiles respectively, $x$-axes represents position $(\mu \mathrm{m})$ while the $y$-axes represent un-normalized field amplitude.

A further examination of the mode profiles in figure 6.2 illustrates that in a 4 layer high dielectric clad configuration, substantial field components exist in the high dielectric medium. In order to fully exploit this property, the high dielectric overlay can be patterned such that regions of high dielectric are replaced with the superstrate material and the pattern repeated in the light propagation direction. One possible arrangement is to configure the overlay into a $1-\mathrm{D}$ grating with a pitch $\Lambda$. The patterning is chosen to go the full depth of the overlay such that the waveguide is formed from connected 3 layer and 4 layer segments. The dominant optical effect of the grating patterned overlay is to reflect the modes that match the Bragg condition $\left(\beta=\frac{l \pi}{\Lambda}\right.$ for $\left.l=1,2, \ldots\right)[66]$. Figure 69 
6.3(top) shows the overlay layer considered and figure 6.3(bottom) shows the spatial Fourier transform obtained based on the periodicity $(\Lambda=0.5 \mu \mathrm{m})$, index of refraction values $(3.480: 1.000)$ and duty ratio $(50 \%)$. The grating is designed to have a dominant spatial wavelength that corresponds with the design wavelength of $1.55 \mu \mathrm{m}$. The harmonics are quite distant from the fundamental and are outside of the wavelength region of interest. If the duty cycle is not properly balanced in space to take into account the value of the effective index of refraction, even harmonics are generated in the spectrum as well as a reduction the fundamental amplitude. These additional frequency components are sufficiently far from the fundamental as to be of no concern for the wavelength range examined. Fourier analysis techniques can also be used to compare different grating configurations. Based on these considerations the square modulated grating was selected as the pattern overlay with the parameters as have been described. 

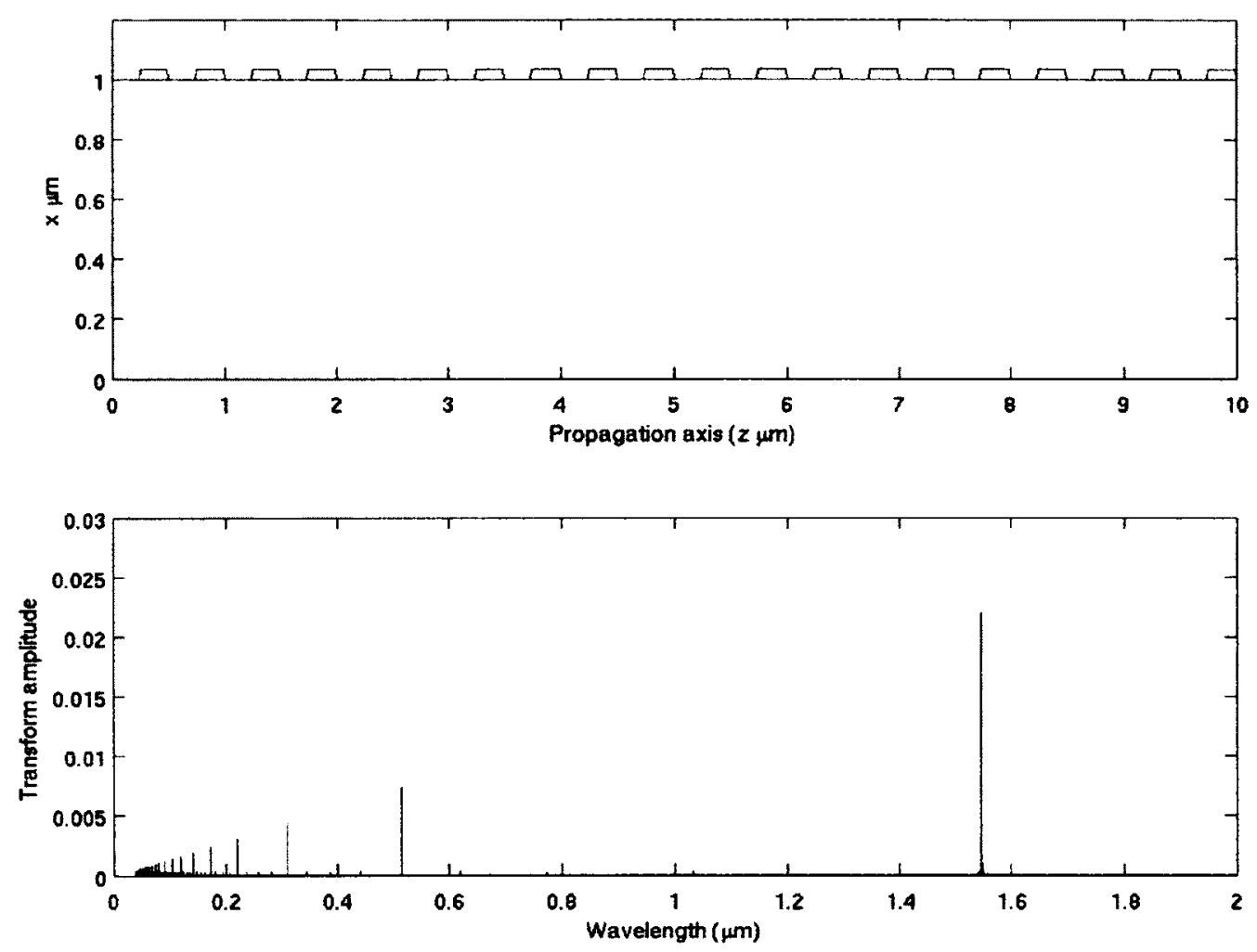

Figure 6.3 Top: $(x, z)$ plane cut through the grating region of the waveguide configuration. Bottom: Spatial Fourier transform of the grating layer demonstrating the fundamental corresponding to the design wavelength of $1.55 \mu \mathrm{m}$.

\subsubsection{Structure details for FDTD}

A 2-D FDTD environment was set up to obtain information on the propagation of light in the high dielectric clad waveguide structures. The $(x, z)$ plane of a typical simulation configuration is shown in figure 5.1 and consists of an input 3-layer segment of $3 \mu \mathrm{m}$ followed by a $20 \mu \mathrm{m}$ long grating and then terminated by a $2 \mu \mathrm{m}$ output 3-layer waveguide. The entire domain was bordered by a $1 \mu \mathrm{m}$ PML with matched dielectric values to reduce reflections from the grid boundary. The $y$-direction was taken as infinite and the entire structure was discretized on a square grid. The input source consisted of a 
short time duration pulse with a profile and field components that matched to those of figure 6.2 for the 3-layer geometry. The source has a spectral extent that covers the wavelengths of interest by centering it at a wavelength of 1.550 with a width $w=0.3340$. Its shape is the discrete-time derivative of a Gaussian as per equation (4.27). The wavelengths are relatively close such that the temporal pulse appears to be about 8 of the central wavelengths in length or about $40 \mathrm{fs}$ in duration. The propagation of short pulses are affected by chromatic dispersion in real devices; however, this source is used to mimic a broadband source for the purpose of simulation. Flux planes were located such that the transmittance and reflectance in various regions of the waveguide could be monitored. As per figure 5.1, the source plane is spaced $2 \mu \mathrm{m}$ from the grating while the reflection and transmission flux planes are $1 \mu \mathrm{m}$ from the grating.

Convergence tests performed indicate that a grid resolution of 160 points per micron are required to resolve the top layer in sufficient detail and that 60000 time iteration steps are necessary to ensure that the input source pulse has propagated the full length of the waveguide and through the flux planes. The simulation results are shown in figure 5.5, for the reflectance and transmittance spectra. The transmittance plot for the wavelength range examined shows the expected Bragg dip characteristic for the design wavelength of $1.55 \mu \mathrm{m}$. At high wavelengths the guided mode does not match the Bragg condition and a high throughput is observed. At lower wavelengths we see a second dip followed by a slow recovery of the transmission for decreasing wavelength. This matches the reflectance plot where the standard transmission dip at the design wavelength is followed by a fairly sharp transition to a pass band. The deviation from a typical Bragg reflector is due to the excitation of both reflected and radiated substrate modes.[72] 72 
Considering these properties in terms of wavelength the waveguide with the patterned overlay can be thought of as a high pass filter with a well-defined cut-off wavelength $\lambda_{c}=1.585 \mu \mathrm{m}$. Wavelengths that are shorter than $\lambda_{c}$ are coupled to backwards propagating modes or to the substrate. The substrate in the case of the waveguide described here is effectively infinite resulting in a continuum of substrate modes.

\subsubsection{Simulation results}

The reflectance and transmittance curves of figure 5.5, were determined for a specific set of design parameters. The following additional simulations provide results for geometries with varying grating length, grating period, overlay thickness, and refractive index.

The results of the reflectance and transmittance for grating lengths of 10,20 , and $30 \mu \mathrm{m}$ are shown in figure 6.4. All other simulation parameters remain as previously provided. For the short length grating the Bragg dip is unresolved with respect to the secondary dip caused by substrate mode conversion. As the length is varied the dips do get resolved and get deeper with grating length.
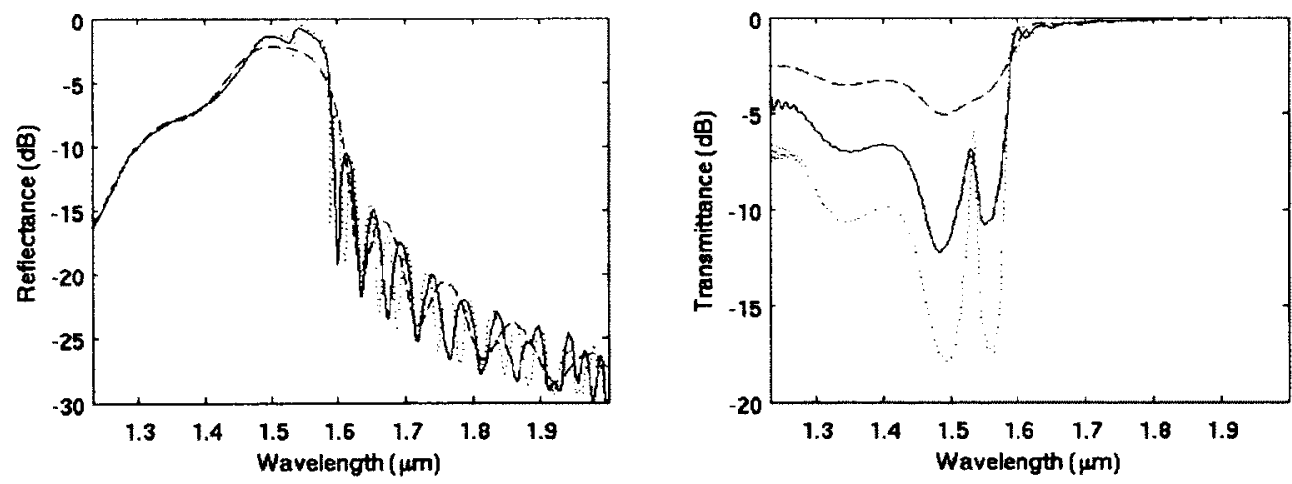

Figure 6.4 Left: reflectance and Right: transmittance for different grating lengths. The solid line represents the standard simulation with a $20 \mu \mathrm{m}$ length, while the dashed and dotted lines represent 10 and $30 \mu \mathrm{m}$ lengths respectively.

73 
In addition, a longer grating length was simulated to further the trends observed. In figure 6.5 the results for $40 \mu \mathrm{m}$ are shown to suggest a continuation of the deepening and narrowing of the transmission dips albeit with a simulation that was run for a shorter duration than would provide a guaranteed accurate result. This result was not published but has been included here to provide an idea of additional research that was conducted to develop a deeper understanding of the structure in preparation for the device considered in the next section.
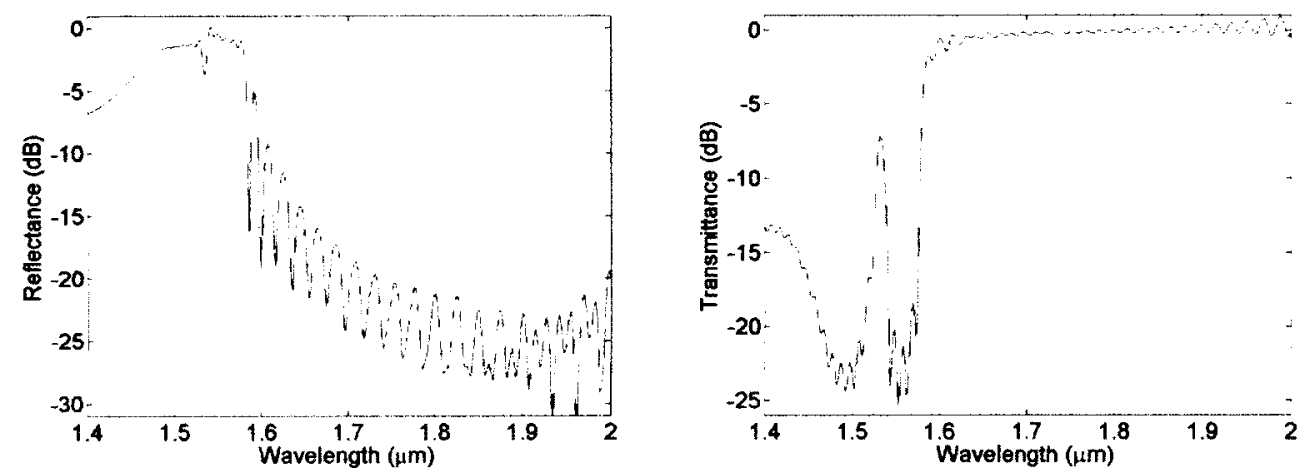

Figure 6.5 Reflectance (left) and transmittance (right) for a grating length of $40 \mu \mathrm{m}$ - note the change in vertical axis.

The grating pitch was determined in preliminary calculations made by the simulation code that would then invoke the FDTD engine. The structure parameters for the 3 layer and four layer waveguide sections would be provided to the simulation code along with the desired Bragg wavelength. The code would solve the field equations at that wavelength and provide the quarter wavelength sectional lengths for the effective index determined for the modes in the 3 and 4 layer sections. The sum of these sectional lengths provide the pitch of the grating. To investigate the effect of pitch on the optical characteristics of the structure we consider changing the pitch from $\Lambda=0.498$ to $\Lambda=$ 74 
0.453 and $\Lambda=0.554$ using the built in code for the calculations. Using a more accurate standalone field equation solver the correct pitch for the initial pitch is $\Lambda=0.495$. Nevertheless, since this is such a small difference the corresponding Bragg wavelengths are still expected at $\lambda_{B}=1.5500, \lambda_{B}=1.4091$, and $\lambda_{B}=1.7222$ as per the built in code. The grating length is kept constant thus the shorter pitch gratings contain more periods. Having more periods strengthens the response of the structure. Figure 6.6 shows the response for changing the pitch. The Bragg dip location is found to follow the calculated value with the secondary dip response following the same level of shift. The reflectance is observed to be stronger for the smaller pitch and weaker for the longer pitch due to increase in the number of periods in the $20 \mu \mathrm{m}$ length grating.
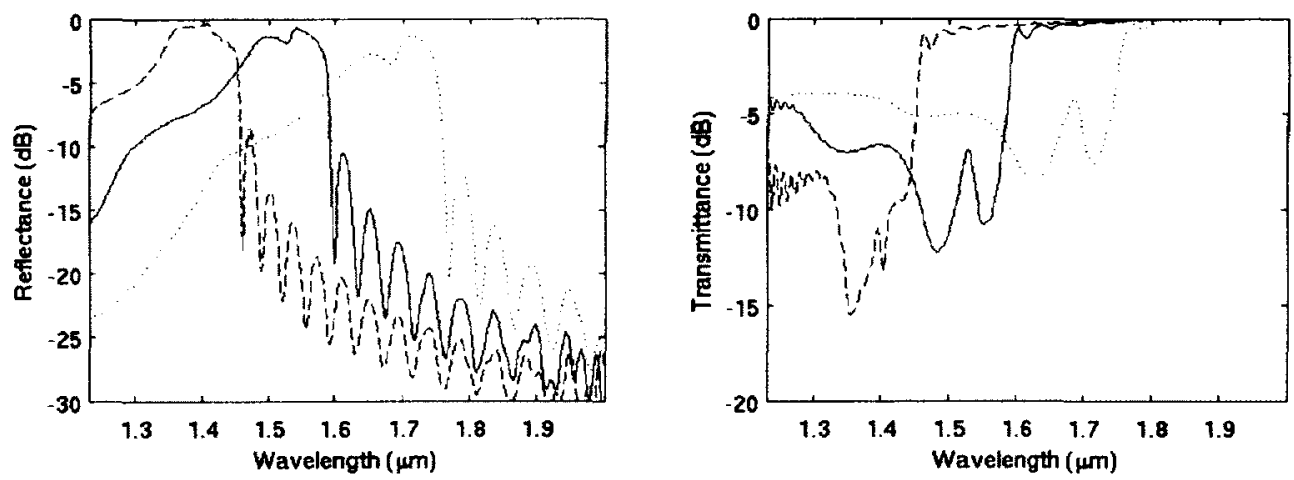

Figure 6.6 Left: reflectance and Right: transmittance for gratings with periods designed for different Bragg wavelengths. The solid line represents the standard simulation with $\lambda_{B}=1.5500$, while the dashed and dotted lines represent $\lambda_{B}=1.4091$ and $\lambda_{B}=1.7222$ respectively.

The response as a function of grating thickness is examined with the constraint that the top layer does not become a single mode waveguide on its own. The three thicknesses explored are $t_{2}=0.0222, t_{2}=0.02845$, and $t_{2}=0.0347$; and the response of 
the structure is shown in figure 6.7. As expected a thicker overlay pulls in more of the mode into its layer and thus a larger response is observed for thicker layers.
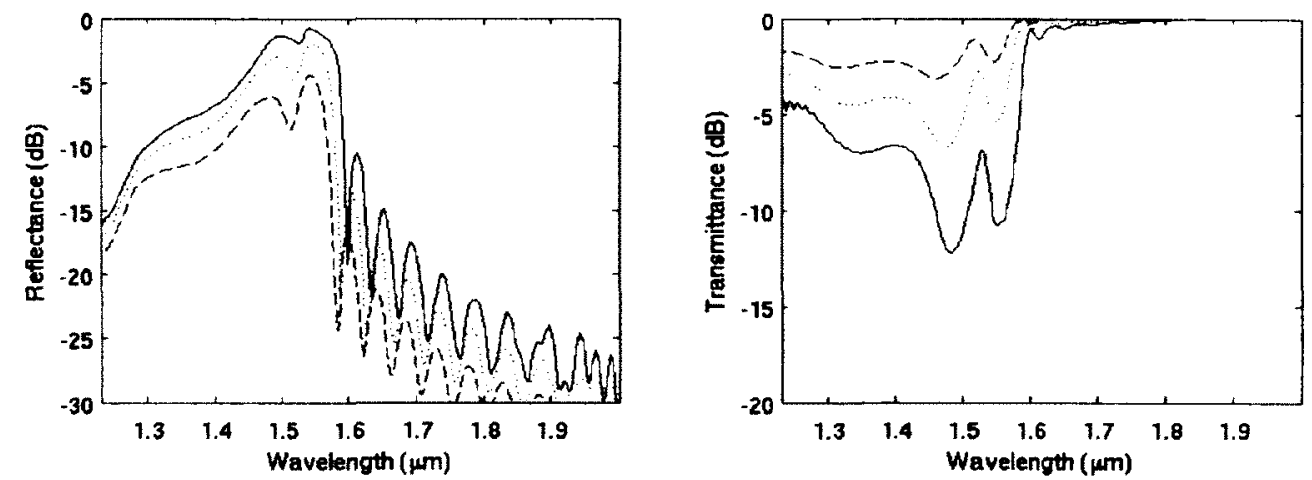

Figure 6.7 Left: reflectance and Right: transmittance for different overlay thicknesses. The solid line represents the standard simulation with $t_{2}=0.0347 \mu \mathrm{m}$, while the dashed and dotted lines represent $t_{2}=0.02222 \mu \mathrm{m}$ and $t_{2}=0.02845 \mu \mathrm{m}$ respectively.

Refractive index change can be accomplished by either changing the overlay material during fabrication or changing it in situ by using any one of many active techniques (e.g. thermo-optic, carrier-injection, ...). The response versus index change is shown in figure 6.8 , where the values for $\mathrm{n}_{2}$ are determined for silicon at room temperature, $300^{\circ} \mathrm{C}$, and $600^{\circ} \mathrm{C}$ with a thermo-optic coefficient resulting in $\frac{d n}{d t}$ of $1.8 \times 10-$ 4/K. Increasing the refractive index of the overlay causes the Bragg dip and substrate related mode coupling to become stronger. The corresponding regions in the transmittance trace get lower. 

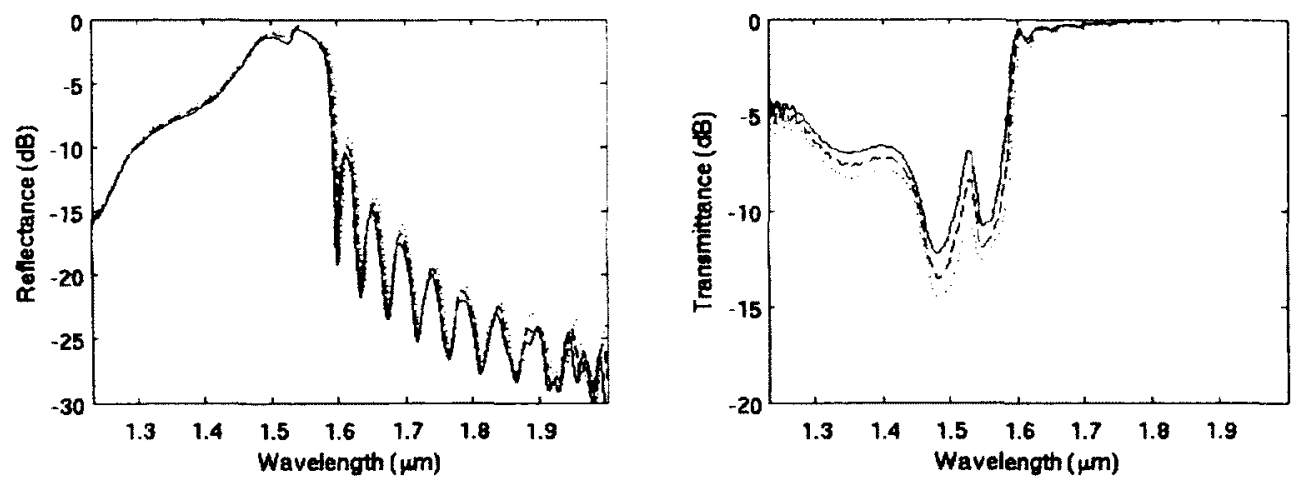

Figure 6.8 Left: reflectance and Right: transmittance for different overlay refractive indices. The solid line represents the standard simulation with $n_{2}=3.480$, while the dashed and dotted lines represent $n_{2}=3.534$ and $n_{2}=3.588$ respectively.

Active devices rely on structures whose response characteristics can be changed after fabrication has been completed. In our structure the in situ modification of the refractive index by heating achieves this. The Bragg dip in figure 6.8 , becomes lower with increased refractive index. Also note that the corner wavelength edge shifts towards lower values with increasing refractive index. The difference in transmittance at a heated state (higher index) versus transmittance at room temperature is plotted in figure 6.9 (left). It is observed that the largest change takes place near the pass-band edge. The second plot in figure 6.9 (right), shows this region enlarged for the corresponding transmittance plot (figure 6.8 right). The shifting of the pass-band edge through a thermal modification of the refractive can be used to modulate an optical signal and thus the high dielectric clad waveguide design can serve as an active device. The time response can be increased by using other non-thermal techniques to change the index of refraction of the silicon layer (carrier injection, non-linear, ...). The observed effect displayed in figure 6.9 can be further increased by optimizing the parameter set for the high dielectric clad 
overlay. Referring to figures 6.4 through 6.8 , the largest Bragg dip is observed for the longest, thickest, highest refractive index overlay and with the smallest pitch. Having the smallest pitch provides more periods for a given length. However, the pitch is normally set for a given design wavelength. The thickness and refractive index of the high dielectric layer must be chosen such that the overall waveguide structure is single mode. This leaves the grating length which can be increased considerably to provide a very sharp and strong response and still provide a device under $100 \mu \mathrm{m}$ in full length.
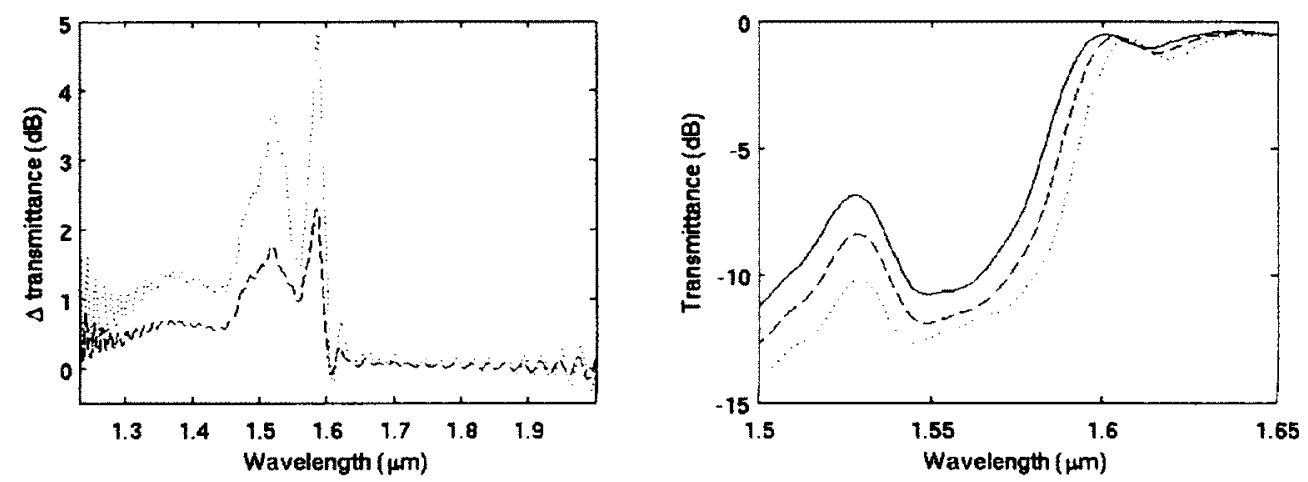

Figure 6.9 Left: change in transmittance. The dashed and dotted lines represent $n_{2}=3.534$ and $n_{2}=$ 3.588 versus the standard simulation respectively. Right: a closer look at transmittance for different overlay refractive indices. The solid line represents the standard simulation with $\mathbf{n}_{2}=3.480$, while the dashed and dotted lines represent $n_{2}=3.534$ and $n_{2}=3.588$ respectively.

\subsubsection{Comparison and performance evaluation}

Examination of the modifications to the optical properties of a waveguide with a patterned thin high dielectric overlay demonstrates that the asymmetric nature of the entire waveguide configuration makes it possible to keep the waveguide mode confined in the original waveguide while enhancing the evanescent wave-overlay interaction. For this thesis structures were developed making use of patterned overlays beginning with the 
development of the underlying structure. The high dielectric contrast of a thin coating of silicon placed on a slab waveguide was examined. The silicon can be polycrystalline in the case that the grain boundaries are on the same order of dimension as the final feature size. Then the field profile with and without the overlay was presented along with the calculated mode overlap. The enhanced extent of the evanescent field in the overlay magnifies the overlay effect on the guided light. This makes it possible to design optical waveguide devices that are directly optical fibre compatible while taking advantage of the high dielectric contrast that exists between the silicon overlay and air. This high contrast structure allows various features to be considered by patterning the overlay layer into many different geometries such as a grating or a photonic crystal. Gratings with high dielectric contrast provide the realization of features not normally present for a moderate index contrast in glass-based integrated optics. In the case of the grating structure considered, the high index contrast allows a much shorter grating which is useful in many cases such as integrated optics. Periodization of the overlay is considered in the frequency domain. The sensitivity of the guided light to variations in grating length, grating pitch, grating thickness, and refractive index of the patterned overlay were analyzed using Finite Difference Time Domain (FDTD) simulations. Transmission and reflection spectrums were obtained from which the aspects to consider for active and passive optical device configurations were explored detailing that the strongest filter effect is found for the longest, thickest, highest refractive index overlay with the smallest pitch. In order to consider active devices the refractive index change could be in situ such that the filter edge can be modulated. Thus possible passive and active integrated optical 
applications include use as a low pass filter as well as for the modulation of optical signals.

\subsection{Design of a silicon overlay glass waveguide sensor}

A glass-based slab waveguide, coated with a thin patterned high dielectric overlay, is configured into a refractive index sensor, as published in [11]. The asymmetric nature of the waveguide configuration is exploited by keeping the mode in the slab waveguide while enhancing the field level in the overlay-superstrate. The sensor's response is examined using the FDTD simulation technique. A sensitivity of up to one part in $10^{5}$ in the index of refraction discrimination is determined. The nature of the sensor enables optical fibre compatibility, requires sub- $\mu \mathrm{L}$ sample volumes and provides a high resolution. Previously proposed sensors which have similar footprints and responsivities include a 13 um sensor providing $90 \mathrm{~nm} / \mathrm{RIU}$ [43] and an 8 um sensor providing 130 nm/RIU[44] which have small non-fibre compatible mode sizes. The researchers of the latter mention a much longer 173 um sensor providing a lower resolution of 33 $\mathrm{nm} / \mathrm{RIU}[45]$. This longer sensor has a larger mode size. They mention the option of using a 10 um plasmon sensor providing $465 \mathrm{~nm} / \mathrm{RIU}$ but with a large $10 \mathrm{~dB}$ loss.

In order to demonstrate the principles involved in this hybrid waveguide configuration we employ a slab waveguide in glass and a silicon cover layer pattemed as a Bragg grating.[9] The functionality is explored by examining the structures performance as an index of refraction sensor sensitized to the superstrate region. The next section presents the design of the sensor. The sensor's performance is then presented.

\subsubsection{Design and objective}


The simplest planar optical structure is the traditional three layer glass slab waveguide (superstrate-waveguide-substrate) as shown in chapter 3 , modified here into a periodic 4 layer structure by adding a thin patterned high dielectric overlay at the superstratewaveguide interface (superstrate-overlay-waveguide-substrate). The optical properties of such structures can be modeled, as shown in chapter 4 , using numerical methods such as the Finite-Difference-Time-Domain (FDTD) $[30,31]$ technique, as shown in chapter 5, provided the thin overlay layer is well resolved in the discretized grid. In this case thermal and wavelength dispersion are not included. The structure analyzed parametrically in the previous section was thinned down to maintain single mode operation and lengthened to maintain a strong response as a liquid refractive index sensor.

\subsubsection{Structure details for FDTD}

The structure under consideration is shown in Figure 6.10. The substrate layer is glass with an index of refraction $\mathrm{n}_{4}=1.500$ and extends to $-\infty$ in the $\mathrm{x}$ direction. The waveguide layer is formed from a $1 \mu \mathrm{m}$ thick slab of index $n_{3}=1.600$. The superstrate layer is typically air of index $n_{1}=1.000$ and extends to $+\infty$. In the operation as a sensor, the superstrate material in the region of the overlay grating layer is changed to the

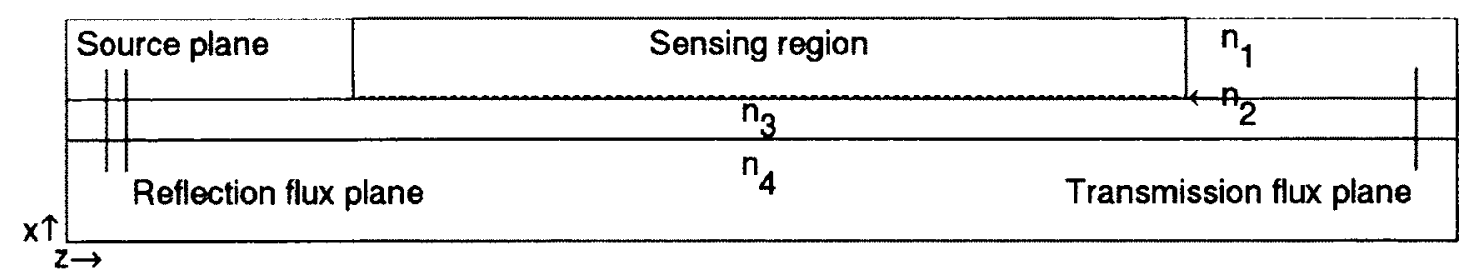

Figure 6.10 Slab waveguide in glass with thin patterned silicon grating overlay. Source, reflection, and transmission planes used in FDTD simulation shown.

81 
material index under consideration. The thin overlay layer, shown as a dashed line, has an index of $n_{2}=3.480$, a thickness of $t_{2}$ dependent on the desired sensing range. Initially when considering liquids consisting of water and glycerol mixtures, the highest target index was that of glycerol (1.4746). For this a thickness of $t_{2}=16.4 \mathrm{~nm}$ was used such that the overall wave guiding structure remains single mode for the highest index fluid. However for the published paper, the application considered sugar solutions with the highest target index as $77 \%$ sucrose (1.465). For this a thickness of $t_{2}=17.5 \mathrm{~nm}$ is patterned as a Bragg grating in the $z$ direction. The grating pitch is designed to meet the Bragg conditions for a wavelength of $1550 \mathrm{~nm}$ when the sensing region contains the highest index material to be sensed. A $40 \mu \mathrm{m}$ grating is used to increase sensitivity. The alternating high and low effective index regions of the grating are each made a quarter wavelength in length which is determined by using the propagation constants of the 3 and 4 layer modal solutions. The sum of a quarter wavelength long section of the three layer waveguide and a quarter wavelength section of the four layer waveguide provides the grating pitch.

The $(x, z)$ plane of the 2-D FDTD environment set up to model the propagation of light in the high dielectric clad waveguide sensor structures is shown in figure 6.10 and consists of an input 3-layer segment of $1 \mu \mathrm{m}$ for the source plus $12 \mu \mathrm{m}$ followed by a 40 $\mu \mathrm{m}$ long grating and is terminated by a $12 \mu \mathrm{m}$ output 3-layer waveguide. The entire domain was bordered by a $1 \mu \mathrm{m}$ PML with matched dielectric values to reduce reflections from the grid boundary. The y-direction was taken as infinite and out of the page. The entire structure was discretized on a square grid at 160 points per micron resolution to ensure suitable structure resolution and minimal numerical dispersion as 82 
confirmed through convergence tests. The input source consisted of a short time duration pulse with a profile and field components matched to those for the 3-layer geometry obtained by solving the field equation for the field polarized in the $y$ direction $\left(E_{Y}\right)$. The multi-wavelength source is defined as the discrete-time derivative of a Gaussian curve as per equation (4.27) with a spectral extent that covers the wavelengths of interest by centering it at a central wavelength of $1550 \mathrm{~nm}$ and with a width $w=0.334$. The source plane was spaced $12 \mu \mathrm{m}$ from the grating input and the detector planes are located $11 \mu \mathrm{m}$ from the grating end. The power reflectance and transmittance, normalized to source output, were computed on each detector plane following the technique presented in [65].

\subsubsection{Simulation results}

The simulated spectra for this structure when pure water is in the sensing region, $n_{1}=$ 1.315 for $25^{\circ} \mathrm{C}[77]$, are shown in Figure 6.11 . In the transmission spectrum, the deepest dip at just under $1550 \mathrm{~nm}$ corresponds to the Bragg wavelength of the grating and the secondary dip at a lower wavelength is associated with power coupling to substrate and radiation modes. A band pass region for wavelengths beyond the Bragg wavelength is also observed. The reflection spectrum is a dual of the transmission spectrum showing peaks where the respective dips are, except that there are also radiated modes which are not accounted for in these spectra as discussed in chapter 5. In the analysis of the sensor the transmission spectra are examined and attention is directed at the Bragg dip's wavelength and depth dependence on the sensing region's index. 

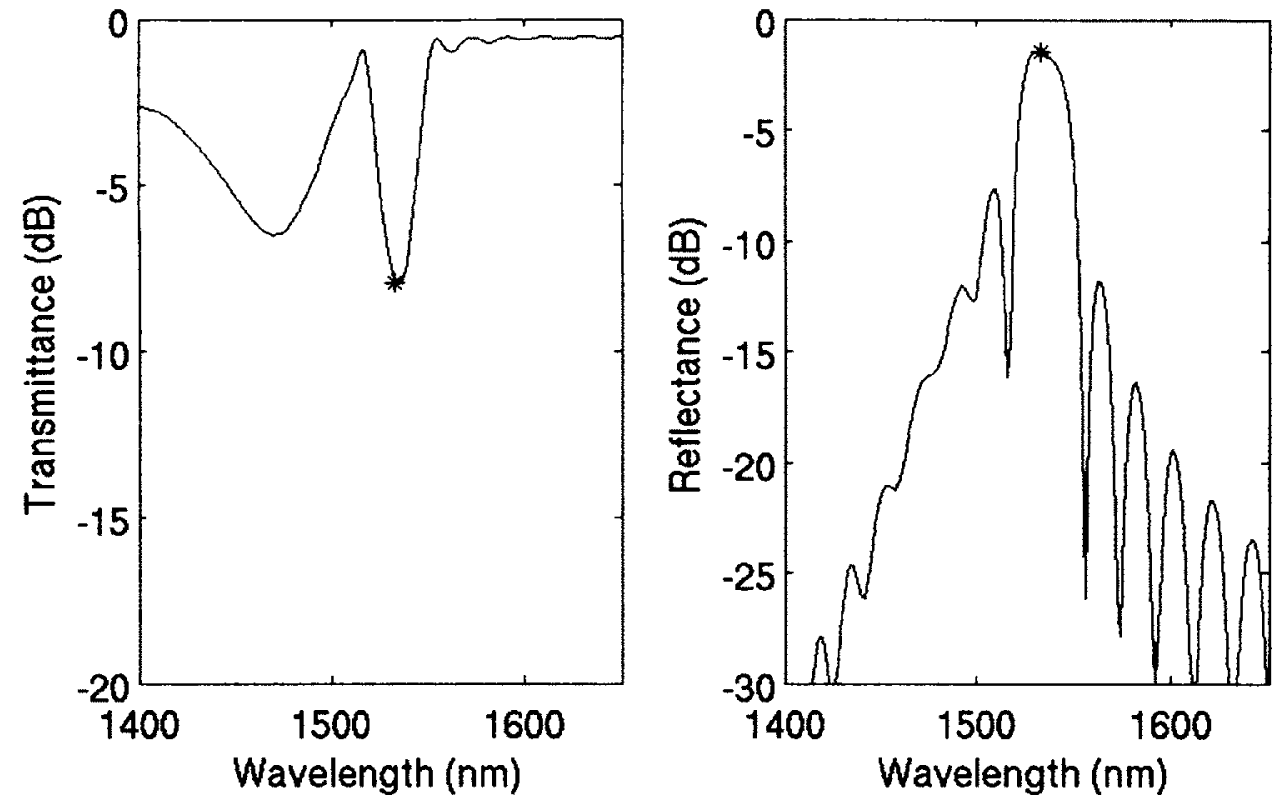

Figure 6.11 Transmittance (left) and reflectance (right) spectra for device design.

The structure's response with pure water in the sensing region is used as a reference for the response with the index of the sensing region increased to a maximum of $n_{1}=1.465$ (a change of 0.15 ) in steps of 0.025 . The index values and range were chosen to be representative of the wavelengths, temperature, and solutes considered. The highest refractive index of 1.465 corresponds to a $77 \%$, or 77 Brix[78], sucrose solution given a refractive index shift similar to that of water due to change of wavelength and the few degree temperature change from the $20^{\circ} \mathrm{C}$ data[79]. As the refractive index in the sensing region is increased the Bragg dip shifts to a longer wavelength as shown in Figure 6.12. In addition, the increasing index has the effect of modifying the effective length of the 4 layer quarter wavelength regions as well as the modal overlap between the cascade of three and 4 layer waveguide regions making up the grating. Since the grating 
is designed for when the sensing region contains the maximum index to be sensed the Bragg dip gets monotonically deeper over the refractive index value sensing range.
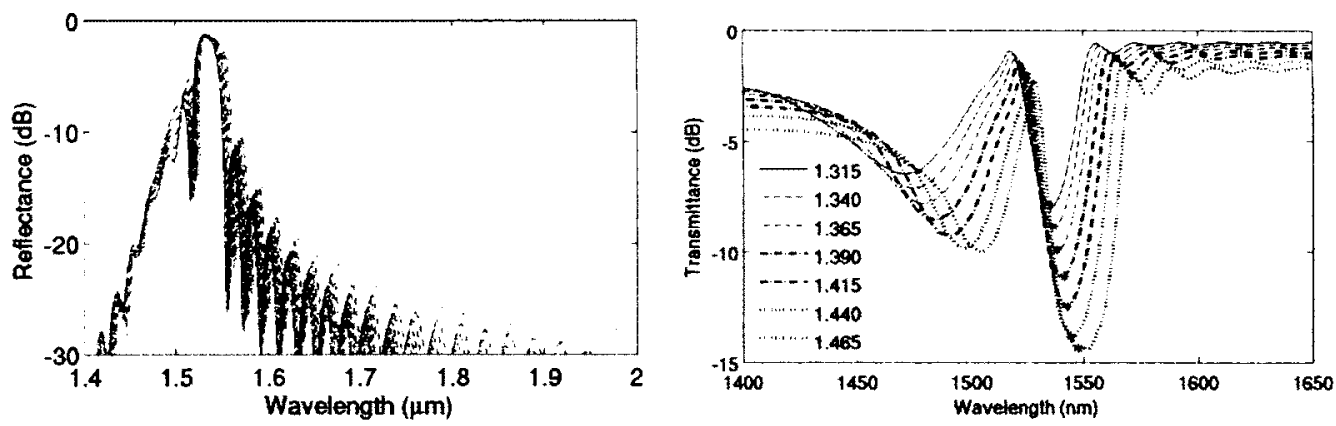

Figure 6.12 Reflectance (left) and transmittance (right) spectra for different cladding refractive indices. The solid line represents the pure water trace with $n_{1}=1.315$, while two each of the dashed, dashed and dotted, and dotted lines represent $n_{1}=1.340,1.365,1.390,1.415,1.440$, and 1.465 respectively. Star points on each trace indicate the location of the Bragg wavelength of the grating.

The sensor's response can be evaluated using two criteria. The first is to track the change in the Bragg dip wavelength and the second is to monitor the change in signal strength for a fixed operation wavelength. The sensor's performance in both modes of operation can be determined from the family of traces in figure 6.12 (right). In order to consider the use of the shift in the Bragg dip versus refractive index, the locations of the minimums in the primary dip are marked with stars in figure 6.12 (right). A wavelength shift of $85 \mathrm{~nm} / \mathrm{RIU}$ is determined and assuming a wavelength resolution of $1 \mathrm{pm}[80]$ a refractive index resolution of $10^{-5}$ is possible. It is also important to consider the full width at half maximum (FWHM) of the signal to determine the relative ease of measurement. A figure of merit (FOM) for sensing has been defined[81] which will be used to help determine 
this influence in equation (6.1) where $m$ is the slope of the change of photon energy for light corresponding to the Bragg wavelength provided in electron volts per RIU.

$$
F O M=\frac{m\left(e V R I U^{-1}\right)}{F W H M(e V)}
$$

The FOM is calculated to be 3.8 which is right in between those presented $(1.6,5.4)$ in the paper that it is defined. More recent phase sensitive collective plasmon resonance sensor work since that paper suggest that a sensitivity of up to $10^{-10}$ RIU is possible.[82] Using the FOM calculation for sensors both based on tilted fiber Bragg gratings and on long period gratings have resolutions on the order of $10^{-4}$ RIU.[83] This highlights the reason that research groups focus on plasmonics. Calculating detection limits from the instrumental resolution limit is simplistic but, in the case of numerical modeling with repeatable results, it does provide a method of comparison. Sensors with much greater wavelength shifts such as $6000 \mathrm{~nm} / \mathrm{RIU}$ have been demonstrated; however, additional factors must be considered such as an example where the detection limit of a ring resonator with a shift of $1000 \mathrm{~nm} / \mathrm{RIU}$ is found to be $3.4 \times$ $10^{-6} \mathrm{RIU}$, poorer than that of a ring resonator with a shift of $25 \mathrm{~nm} / \mathrm{RIU}$ for which it is found to be $1.2 \times 10^{-6}$ RIU.[84] In real systems, measurements can be modeled using a standard deviation approach. The limit of detection for chemical sensors can be determined by standard deviations at low concentration and by using a calibration curve.[85]

When the output intensity is monitored directly, it is instructive to use the pure water trace as a reference and then determine the change in output intensity as a function of wavelength and sensing region index relative to the chosen reference. The families of 86 
traces in figure 6.13 represent change in output intensity derived from the data of figure 6.12(right). The maximum of the change in transmittance is used to start the sensor wavelength selection. Next the response for wavelengths slightly above and below this were compared to find a wavelength with the strongest change in response matching the center of the index range considered.

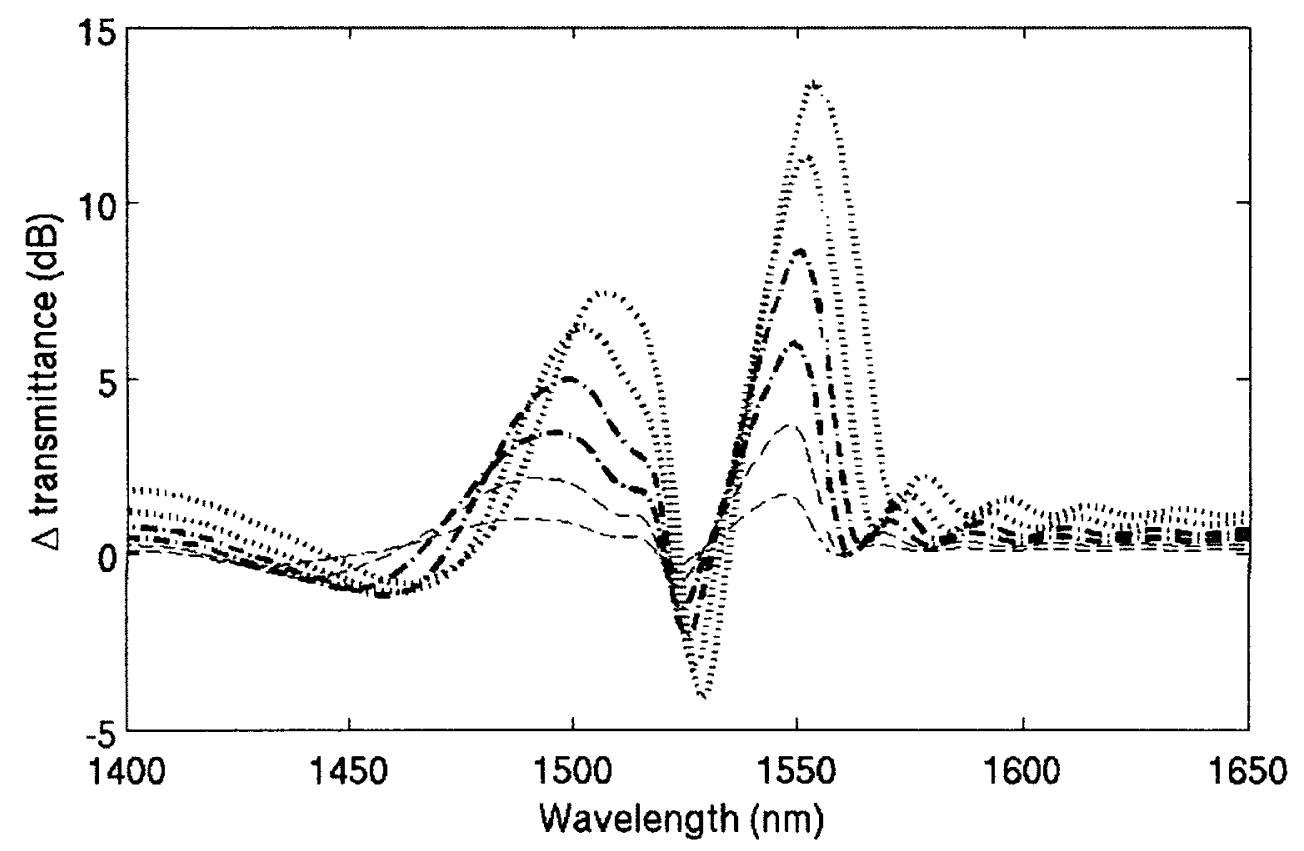

Figure 6.13 Change in transmittance. Two each of the dashed, dashed and dotted, and dotted lines represent $n_{1}=1.340,1.365,1.390,1.415,1.440$, and 1.465 versus the $n_{1}=1.315$ simulation respectively.

The sensor response for a wavelength of $1556 \mathrm{~nm}$ is shown in figure 6.14. Depending on the desired sensing index range, the sensing wavelength can be optimized for the maximum slope and linearity for that range. Since amplitude detection units are able to resolve $0.1 \%$ intensity changes[86] this provides a sensitivity of being able to 
detect changes as low as $1.8 \times 10^{-4}$ in refractive index for a $40 \mu \mathrm{m}$ sensor. Fabrication roughness can reduce the sharpness and strength of a grating dip which can be compensated by increased length.[32]

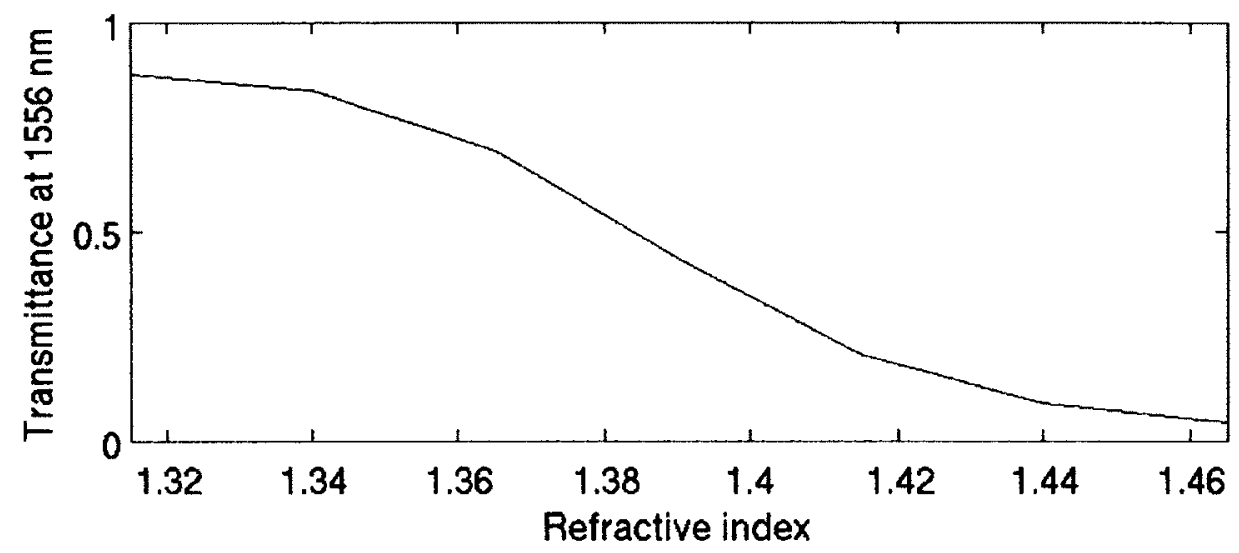

Figure 6.14 Sensor normalized power response for source wavelength of $1556 \mathrm{~nm}$.

A possible device configuration in addition to a power level output response from a single sensor would be to have the source branched into several gratings with slightly modified pitches such that the output of each would be detectable at different refractive indices providing a digital readout.

\subsubsection{Comparison and performance evaluation}

A refractive index sensor has been presented based on the optical properties of a slab waveguide coated with a patterned thin high dielectric overlay. The hybrid nature of the entire waveguide configuration makes it possible to keep the waveguide mode in the slab waveguide compatible to optical fibre while enhancing the evanescent wave-overlay interaction using the high dielectric. The sensitivity of the guided light to variations in the cladding refractive index was examined using FDTD simulations and transmission 
spectra are obtained for various superstrate refractive indices. The response of the sensor is examined using both the change in the Bragg dip wavelength and transmitted signal strength and is shown to provide the more accurate index resolution of up to one part in $10^{5}$ when tracking the Bragg dip with a figure of merit in the range of plasmon sensors. 


\section{Conclusions}

It has been found that high refractive index overlays can provide the benefits of high refractive index contrast to an optical fibre compatible waveguide platform. The size of the proposed devices would allow several sensors to interrogate a single applied droplet. State of the art devices with either similar insertion loss, compactness, or sensitivity are referenced demonstrating that this work is significant in that it combines all of these characteristics into a single structure. The modal theory was used to explain the coupling characteristics and to be able to calculate field amplitude distributions for the structure. The reasoning for the use of FDTD for simulations was following by a description of the method including sample equations and results. Novel devices and their tuning were then presented which this chapter summarizes and then presents possible directions for future work.

\subsection{Novel findings}

This work produced many novel findings which were published based on structures with thin high refractive index overlays. Although the emphasis of this work was on single mode structures, multimode structures were investigated alongside. They were found to provide methods of coupling to a high refractive index layer via an MMI like structure which could also be used for devices such as sensors. Close analysis of the four layer modes demonstrated clearly the unique scenario of the peak of a mode entering the high dielectric overlay prior to its effective index exceeding the core guiding layer material's refractive index. This transition region is the suggested operating point for the patterned overlay structures. These findings were published in concert with this work. 
In the effort to model the proposed structure and the related devices, approaches were compared. A matrix-based technique and a perturbation approach were compared to FDTD simulations. It was discovered that the matrix approach provides reasonably accurate predictions of the strength and location of the Bragg dip until the refractive index of the overlay approaches and exceeds that of silicon. The perturbation approach was found to predict a reasonable value for the strength of the Bragg dip for overlays with refractive index approaching and exceeding that of silicon. The FDTD simulations demonstrated that there is a range of refractive index for this structure in which the location of the Bragg dip in wavelength does not change appreciably for changes in overlay refractive index allowing for material tolerance which can be for fabrication error, for carrier injection, and for doping. FDTD is recommended as the preferred approach. In addition, these simulations enabled the production of the appended catalog of valuable results.

When addressing the FDTD computation engine the results of preliminary computations were discussed. A modeling issue was the duration of simulations and the reduction of the problem space and use of a multi-wavelength pulse along with parallel computing provided a solution to this. In the preliminary results from the patterned high refractive index overlay, in addition to the primary Bragg dip, a secondary dip was formed by the coupling to a continuum of substrate modes.

The research focused on providing a published parametric analysis of a periodic thin overlay. Structures making use of this were found to provide opportunities for compact devices and a demonstration of such a use of this platform for integrated optic solutions was provided. The asymmetric nature of the entire waveguide configuration 91 
makes it possible to keep the waveguide mode confined in the original waveguide while enhancing the evanescent wave-overlay interaction. The silicon can be polycrystalline in the case that the grain boundaries are on the same order of dimension as the final feature size. Gratings with high dielectric contrast provide the realization of features not normally present for a moderate index contrast in glass-based integrated optics. Transmission and reflection spectrums were obtained from which the aspects to consider for active and passive optical device configurations were explored detailing that the strongest filter effect is found for the longest, thickest, highest refractive index overlay with the smallest pitch. In order to consider active devices the refractive index change could be in situ such that the filter edge can be modulated. Thus possible passive and active integrated optical applications include use as a low pass filter as well as for the modulation of optical signals. These applications are relevant to the telecom industry in the current push for inexpensive integrated optic FTTx solutions. In these solutions it would be possible to leverage the optical fibre compatibility of the structure. At the same time, these solutions could use the ability of the researched platform to provide a high dielectric contrast in order to make use of photonic crystal devices which would expand on the one dimensional Bragg grating examined.

The structure was further investigated for a refractive index sensor application for which the results were then published. The response of the sensor is examined using both the change in the Bragg dip wavelength and transmitted signal strength and is shown to provide the more accurate index resolution of up to one part in $10^{5}$ when tracking the Bragg dip with a figure of merit in the range of plasmon sensors. This platform shows 
that it is possible to combine high refractive index contrast compact devices with fibre compatible waveguides.

\subsection{Future work}

It would be most interesting to take the devices presented to production. Additional sensor analytes such as those which have an imaginary component to their refractive index could be considered. Additional methods of interrogating the sensor could also be considered such as with a source than performs a wavelength scan. In order to prepare for this the fabrication of prototypes of the investigated designs would be essential. Alongside production process flow development, staying up to date with 3-D simulation opportunities to investigate additional device designs would be beneficial. It would help both by having simulation runs that could be attempted in the background during other work and by providing insight into how channel waveguides could form several integrated devices. 


\section{Appendices}

\section{Appendix A Material properties}

When using a refractive index sensor for fluids, it is important to be aware of the properties of the fluids. In the case of solutions, the refractive indices of the fluids change with concentration and with temperature. Solutions also have a temperature dependent saturation point. In terms of biosensors, it is possible that only the refractive index near an interface changes. Figure A.1 provides a chart of refractive index versus concentration for two sugars, glycerol and salt. Sucrose solutions were used in main text (chapter 6) as the representative fluid for sensing. The figure shows that a sensor that can detect refractive index change can be used to measure the properties of several different

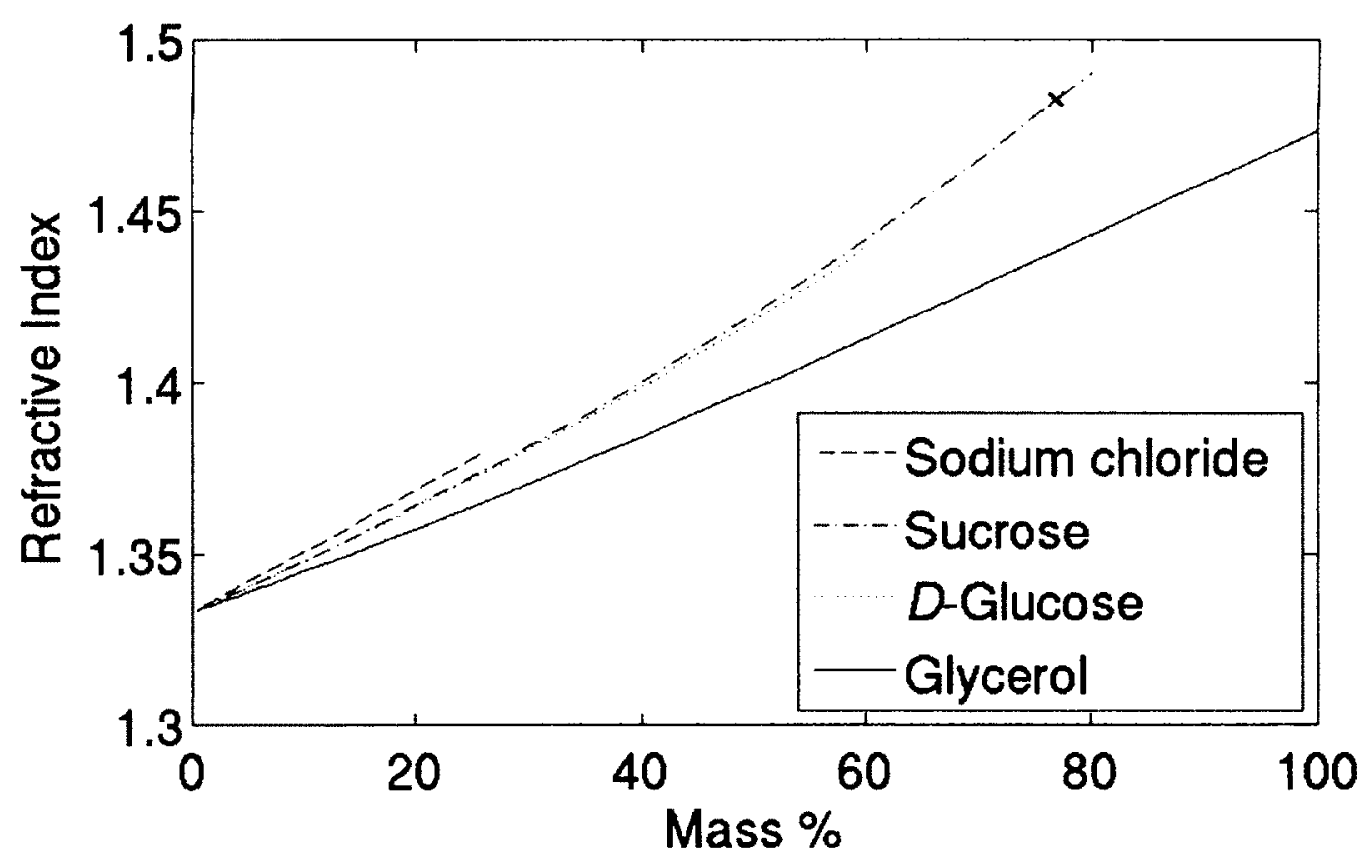

Figure A.1 Refractive index versus mass percentages for two sugars, glycerol, and salt showing similar linear trends. 
materials in a similar way. The sensor requires material specific calibration. It is important to be aware of conventions and terminology used by authors especially when considering concentrations of solutions. Mass percentage of the solute is taken with respect to the total mass of the solution. However, it is possible to find instances where the solute is compared to the solvent in volume, which can be quite different. In this work, water as the solvent has a mass of approximately $1 \mathrm{~kg}$ per cubic decimetre depending on temperature, atmospheric pressure, purity, etc. which means that certain sets of units are approximately equivalent and similar enough for this work. In the body of this work, the refractive index of water is taken to be 1.315.[77] However, this appendix discusses a different set of published data and thus it is taken to be 1.333.[80] The refractive index of water is increased with salt concentration up to the brine saturation point in figure A.1. The slope of the chart demonstrates that the saline $\Delta \mathrm{n}$ is fairly linear with $\Delta \mathrm{n}=1.9225 \times 10^{-4} \mathrm{~g} / \mathrm{kg}$. Sugars are considered since they are also a possible analyte. D-glucose $\Delta \mathrm{n}=1.4 \times 10^{-4} \mathrm{~g} / \mathrm{kg}$ around room temperature, thus as the sugar concentration changes from $0.1 \%$ to $0.3 \%$ (the range for diabetes using the definition $\mathrm{g} / 100 \mathrm{~mL}=\%$ concentration), the index of refraction changes from 1.3334 to 1.3337.[87]. Sucrose is used in this work and in similar work in the food industry where concentration percentages are referred in degrees Brix[78]. In order to compare to the literature, glycerol is also charted. At $100 \%$ glycerol, the refractive index $n$ is $1.4746 .[80]$ 


\section{Appendix B Discussion regarding extending analysis to 3-D}

This appendix discusses approaches taken to extend the modeling of the structure described by this work in 3-D. Computing has become powerful so quickly[88] that it was possible to simulate the two dimensional structures in this work. The primary obstacle to widespread use of accurate three dimensional modeling for optics at this point is the amount of time it takes to complete a simulation. Iterative development of 2-D structures can be done with some insight, [89] however, a reasonable amount of time for a single simulation is still considered to be under 16 hours. Parallel processing provides a way of increasing computational throughput. Embarrassingly parallel processing[90] is used in cases where very little communication needs to take place between tasks which are executing. An example of this is for the comparison of two different optical structures. Instead of running a simulation of the first structure and then running the second structure on the same computer to compare results, the two structures could be run at the same time on different computers and then the results could be compared. On the other end of the spectrum, many modern software programs[91] will compile sections of for loops, even if originally written as a serial program, into tasks that can be executed by several processor cores, processors, or computers at the same time. Although this does speed up the amount of time that it takes to get a final result, Amdahl's law[92] states that there are diminishing returns on adding additional processors. Amdahl's law's accuracy has been called into question[93] and should new paradigms be able to improve performance of extensively scaled, systems it will be a boon to simulations similar to 
those completed for this work. A secondary obstacle to simulation of 3-D structures at high resolution is the memory required to store the field values at each point.

\section{B.1 Parallel code execution}

In a single optical structure that is segmented (or meshed) into smaller regions, it is often the case that each region can be solved independently but it depends on its neighbours for the initial values for each computational step. Thus for each iteration, there is an amount of communication that needs to take place between tasks which are executing. The amount of communication and the amount of necessarily serial execution is subtracted as a percentage of the program which cannot be parallelized. Developing code to maximize the potential for parallelization can considerably add to development time itself and tools have been developed to aid with this.[94] The primary c++ software library that has been used for the work herein takes optimization of segmenting for parallelization into account.[30] The software developers used the Debian GNU/Linux operating system environment making it straightforward to use on the popular Debian-derived Ubuntu distribution. If such an environment is not available, it is possible to run simulations via a web browser interface on nanoHUB.org.[95] It is also possible to use the Cygwin package[96] which makes tools available in Unix available on a computer using Microsoft Windows as an operating system. Each of these approaches was tested and found to be lacking in compatibility with running a $\mathrm{c}++$ described electromagnetic simulation in parallel in an effective manner. The developers have moved on to using a Scheme scripting language front end while some users have developed a Python scripting based front end. Using these scripting language front ends make it possible to access the 
library and develop simulation code more quickly but disconnect the user from the core software. The Scheme front end was tested but having a native language connection to the core code allows understanding of functionality and communication with developers of alternative software. It also provides stronger control over automatic and default behaviour of the software.

\section{B.2 Cluster use}

Three Beowulf cluster[97] options were investigated. The first option was to use the networked computers in our research lab. This served as a trial configuration to mimic our Department of Electronics (DOE) network. The second option was to use the DOE network itself, which at the time had 5 nodes each with 8 cores and 9 GB RAM available at off peak times. Our department uses RedHat-derived CentOS since the widely used Cadence software Canadian Microelectronics Corporation's (CMC Microsystems') licensing scheme provides compatible binaries for it. The ability to mimic the DOE network and troubleshoot with the assistance of the software support forums made it a slightly less arduous undertaking. The third option was the use of the High Performance Computing Virtual Laboratory (HPCVL) funded by the Canada Foundation for Innovation's (CFI's) National Platform Fund (NPF) whose collaborators provide access to machines typically with more than $1 \mathrm{~GB}$ of memory per core and with up to 39,168 cores.[98] The access fee for a small research group is $\$ 2,000$ per annum which covers the use of 200 threads or processes for $50 \%$ of the time on a combination of the clusters. The access fee for larger research groups is $\$ 5000$ per annum for the use of up to 900 threads for 6 weeks prior to entering into special fee territory. In order to test the 
suitability of the system for this work the software needed to be installed with the appropriate dependencies accounted for. The experience of supporting the DOE network install provided the required ability to respond to challenges that arose. Through the use of a trial account it was discovered that jobs requiring many cores had low priority and thus days could pass before they were run. In cases where a job required extensive memory it would crash if at some point during its run the required memory was not available. These circumstances give some sense of the added complexity required from a coding perspective to mitigate problems arising from using a shared resource. For the networked computers in our research lab, $8 \mathrm{~GB}$ of consumer level DDR2 was found to cost just under $\$ 300$ at the time of inquiry. For the specific optical structure simulated, the most effective approach was using the departmental network until it became unsupported and thus unstable. Follow-up simulations were completed using the networked computers in our lab. In order to be able to predict both the amount of time a simulation would take and the amount of installed memory required, simulations were monitored and empirical equations were formed. For single threaded simulation it is possible to calculate the memory usage by considering the allocation by the software running on specific hardware. However, empirical equations were used to take into account additional memory consumption as problems were scaled across several nodes.

The 2-D performance was considered in order to be able to determine the limitations of the first two cluster arrangements. Computation time depends on the size of the simulation region as well as the amount of time for results to converge for the specific structure. In chapter 6 the number of time steps used for each structure was provided and using a very general rule of 1 second per time step for these structures provides a rough 99 
idea of the simulation time. When simulations exceeded several days, the likelihood of them completing successfully reduced to the point where a month long simulation was unlikely to complete. One such simulation did complete but the remaining attempts experienced power outages, network changes, etc. A clearer limit that was hit is the memory capacity of the clusters. The empirical estimation equation (B.1) was found to be accurate for both 2-D and 3-D simulations.

$$
R A M(G B)=\left(\frac{\text { number of simulation points }}{1.1 \times 10^{6}}\right)^{0.82}
$$

The 3-D performance was considered for the second and third cluster arrangements with the hope that the HPCVL would be able to overcome both the speed and memory obstacles. Unfortunately the performance of these two clusters was found to be very similar as shown in figure B.1. The lowest horizontal axis value corresponds to a $3 \mu \mathrm{m}$ by $3 \mu \mathrm{m}$ cross section structure that is $10 \mu \mathrm{m}$ long at a resolution of 10 points $/ \mu \mathrm{m}$ while the highest horizontal axis value corresponds to a $7 \mu \mathrm{m}$ by $7 \mu \mathrm{m}$ cross section structure that is $20 \mu \mathrm{m}$ long at a resolution of 40 points $/ \mu \mathrm{m}$. Both of these are relatively small numbers of simulation points considering that a resolution of approximately 160 points $/ \mu \mathrm{m}$ would likely be required making this a future endeavour. 


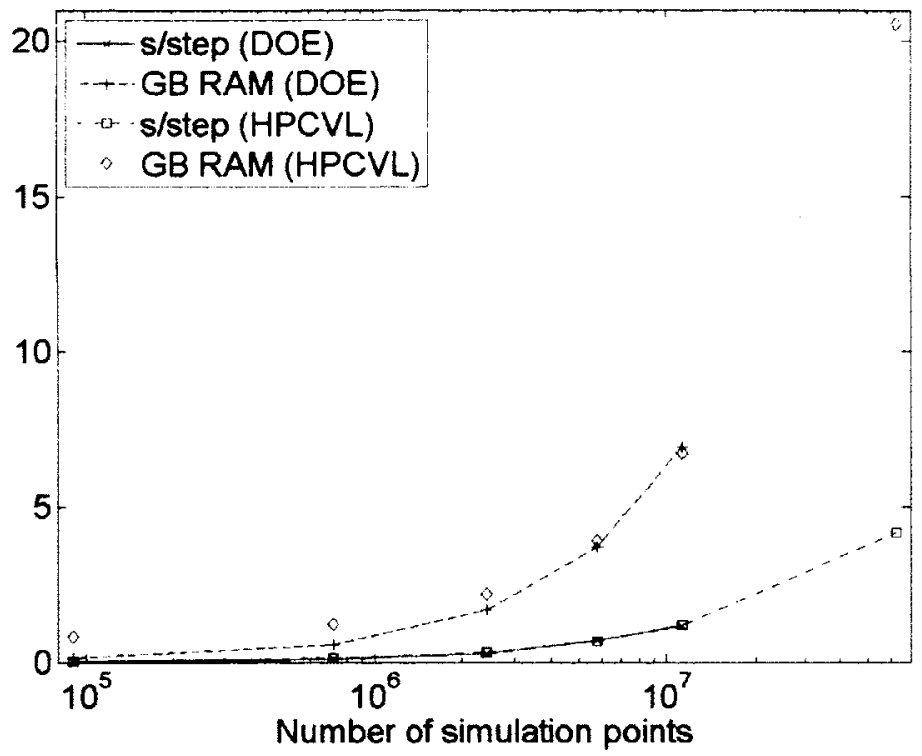

Figure B.1 Number of seconds per simulation time step and GB of RAM required for simulations as the number of simulation points increases.

\section{B.3 Expected response for channel structure}

In concert with the results discussed in appendix D, a 2-D FDTD dielectric evolution simulation engine has been used[61] for a channel structure. The technique works for unidirectional wave propagation by introducing the out of plane propagation constant for a 2-D cross-section of the waveguide. The dielectric profile can be slowly changed to correspond to the environment encountered as the wave front propagates along the waveguide. This can be used for the tapers discussed in appendix D but not for the other structures where multiple reflections result in additional forward travelling wave components. Increased losses are observed for the channel configuration when compared to the slab but the results indicate that the structures can achieve significant coupling in the channel environment as per the slab waveguide configurations. In the full 3-D FDTD 
simulations that ran successfully, it was found that the sharpness of the features in the spectral responses was reduced but retained the shape observed in the 2-D simulation results. Due to the additional degree of freedom for the quasi-TE modes provided by the 3-D environment, the spectral response was also shifted towards longer wavelengths corresponding to the longer effective length of features. 


\section{Appendix C Catalogue of simulations}

This appendix catalogues the majority of the numerous simulations that were run in addition to the simulations discussed in depth in the body of this thesis. Each simulation catalogued generated a summary of results chart along with the data files as shown in the examples in C.3. Unless otherwise noted, the simulations utilized a PML of $1 \mu \mathrm{m}$ surrounding the simulation domain and a $1 \mu \mathrm{m}$ waveguide as described in section 4.4 . In the propagation direction $1 \mu \mathrm{m}$ was used each for the source, flux planes, and buffer distance from the structured overlay region. $7 \mu \mathrm{m}$ was used in the transverse direction. Some simulations are duplicated for various reasons such as the use of different platforms to compare execution time, in order to compare results with different decayed remaining energy in the structure, in order to use different sources, and in order to generate specific field amplitude intensity distribution plots since the data for them is too cumbersome to save in entirety.

\section{C.1 Preliminary simulations}

This set of simulations duplicated and extended results found in previous work including convergence testing in order to confirm the operation of the simulation engine and develop ideas respectively. The simulation run cataloging began once the preliminary ground work and methodology was established. In order to determine the effects of nonperiodic transitions simulations listed in tables C.1, C.2, C.3, and C.4 were run. An additional comparison was the evolution of the structure towards the better known relief grating through the simulations listed in tables C.5 and C.6. 
Table C.1 Periodic overiay simulations.

\begin{tabular}{|c|c|c|c|c|c|}
\hline $\begin{array}{c}\text { Resolution } \\
(\text { points } / \mu \mathrm{m})\end{array}$ & $\begin{array}{c}\text { Overlay } \\
\text { refractive } \\
\text { index }\end{array}$ & $\begin{array}{c}\text { Overlay } \\
\text { thickness } \\
(\mu \mathrm{m})\end{array}$ & $\begin{array}{c}\text { Overlay } \\
\text { length } \\
(\mu \mathrm{m})\end{array}$ & $\begin{array}{c}\text { Bragg design } \\
\text { wavelength } \\
(\mu \mathrm{m})\end{array}$ & $\begin{array}{c}\text { Duration of } \\
\text { simulation }\end{array}$ \\
\hline 40 & 3.6 & 0.032 & 20 & 1.55 & $\begin{array}{c}752 \mathrm{~s} \\
60000 \text { time steps }\end{array}$ \\
\hline 80 & 3.6 & 0.032 & 20 & 1.55 & $\begin{array}{c}2416 \mathrm{~s} \\
60000 \text { time steps }\end{array}$ \\
\hline
\end{tabular}

Table C.2 Simulations of a three layer waveguide terminated with a four layer exit section.

\begin{tabular}{|c|c|c|c|c|c|}
\hline $\begin{array}{c}\text { Resolution } \\
(\text { points } / \mu \mathrm{m})\end{array}$ & $\begin{array}{c}\text { Overlay } \\
\text { refractive } \\
\text { index }\end{array}$ & $\begin{array}{c}\text { Overlay } \\
\text { thickness } \\
(\mu \mathrm{m})\end{array}$ & $\begin{array}{c}\text { Overlay } \\
\text { length } \\
(\mu \mathrm{m})\end{array}$ & $\begin{array}{c}\text { Bragg design } \\
\text { wavelength } \\
(\mu \mathrm{m})\end{array}$ & $\begin{array}{c}\text { Duration of } \\
\text { simulation }\end{array}$ \\
\hline 40 & 3.6 & 0.032 & 20 & 1.55 & $\begin{array}{c}2242 \mathrm{~s} \\
60000 \text { time steps }\end{array}$ \\
\hline 80 & 3.6 & 0.032 & 20 & 1.55 & $\begin{array}{c}7804 \mathrm{~s}=\sim 2.2 \mathrm{~h} \\
60000 \text { time steps }\end{array}$ \\
\hline
\end{tabular}

Table C.3 Simulations using a four layer lead in and a three layer waveguide for the remainder of the structure.

\begin{tabular}{|c|c|c|c|c|c|}
\hline $\begin{array}{c}\text { Resolution } \\
(\text { points } / \mu \mathrm{m})\end{array}$ & $\begin{array}{c}\text { Overlay } \\
\text { refractive } \\
\text { index }\end{array}$ & $\begin{array}{c}\text { Overlay } \\
\text { thickness } \\
(\mu \mathrm{m})\end{array}$ & $\begin{array}{c}\text { Overlay } \\
\text { length } \\
(\mu \mathrm{m})\end{array}$ & $\begin{array}{c}\text { Bragg design } \\
\text { wavelength } \\
(\mu \mathrm{m})\end{array}$ & $\begin{array}{c}\text { Duration of } \\
\text { simulation }\end{array}$ \\
\hline 40 & 3.6 & 0.032 & 20 & 1.55 & $\begin{array}{c}2238 \mathrm{~s} \\
60000 \text { time steps }\end{array}$ \\
\hline 80 & 3.6 & 0.032 & 20 & 1.55 & $\begin{array}{c}7838 \mathrm{~s}=-2.2 \mathrm{~h} \\
60000 \text { time steps }\end{array}$ \\
\hline
\end{tabular}

Table C.4 Simulations using a $10 \mu \mathrm{m}$ four layer section sandwiched in between matching three layer $20 \mu \mathrm{m}$ sections.

\begin{tabular}{|c|c|c|c|c|c|}
\hline $\begin{array}{c}\text { Resolution } \\
(\text { points/ } \mu \mathrm{m})\end{array}$ & $\begin{array}{c}\text { Overlay } \\
\text { refractive } \\
\text { index }\end{array}$ & $\begin{array}{c}\text { Overlay } \\
\text { thickness } \\
(\mu \mathrm{m})\end{array}$ & $\begin{array}{c}\text { Overlay } \\
\text { length } \\
(\mu \mathrm{m})\end{array}$ & $\begin{array}{c}\text { Bragg design } \\
\text { wavelength } \\
(\mu \mathrm{m})\end{array}$ & $\begin{array}{c}\text { Duration of } \\
\text { simulation }\end{array}$ \\
\hline 80 & 3.6 & 0.032 & 10 & 1.55 & $\begin{array}{c}13272 \mathrm{~s}=-3.7 \mathrm{~h} \\
60000 \text { time steps }\end{array}$ \\
\hline 80 & 3.6 & 0.032 & 10 & 1.55 & $\begin{array}{c}19750 \mathrm{~s}=-5.5 \mathrm{~h} \\
60000 \text { time steps }\end{array}$ \\
\hline
\end{tabular}


Table C.5 Simulations with three layer lead in and lead out waveguide sections.

\begin{tabular}{|c|c|c|c|c|c|}
\hline $\begin{array}{c}\text { Resolution } \\
(\text { points/ } \\
\mu \mathrm{m})\end{array}$ & $\begin{array}{c}\text { Overlay } \\
\text { refractive } \\
\text { index }\end{array}$ & $\begin{array}{c}\text { Overlay } \\
\text { thickness } \\
(\mu \mathrm{m})\end{array}$ & $\begin{array}{c}\text { Overlay } \\
\text { length } \\
(\mu \mathrm{m})\end{array}$ & $\begin{array}{c}\text { Bragg } \\
\text { design } \\
\text { wavelength } \\
(\mu \mathrm{m})\end{array}$ & $\begin{array}{c}\text { Duration of } \\
\text { simulation }\end{array}$ \\
\hline 40 & 1.6 & 0.025 & 185 & 1.55 & n/a \\
\hline 160 & 3.6 & 0.032 & 20 & 1.55 & $\begin{array}{c}7505 \mathrm{~s}=2.1 \mathrm{~h} \\
60000 \text { time steps }\end{array}$ \\
\hline 120 & 3.6 & 0.032 & 20 & 1.55 & $\begin{array}{c}4555 \mathrm{~s} \\
60000 \text { time steps }\end{array}$ \\
\hline 80 & 3.1 & 0.046 & 20 & 1.55 & $\begin{array}{c}2351 \mathrm{~s} \\
60000 \text { time steps }\end{array}$ \\
\hline 80 & 2.6 & 0.0734 & 20 & 1.55 & $\begin{array}{c}2325 \mathrm{~s} \\
60000 \text { time steps }\end{array}$ \\
\hline 80 & 2.1 & 0.145 & 20 & 1.55 & $\begin{array}{c}2342 \mathrm{~s} \\
60000 \text { time steps }\end{array}$ \\
\hline 80 & 1.6 & 0.721 & 20 & 1.55 & $\begin{array}{c}2245 \mathrm{~s} \\
60000 \text { time steps }\end{array}$ \\
\hline 80 & 1.6 & 0.721 & 30 & 1.55 & $\begin{array}{c}2782 \mathrm{~s} \\
60000 \text { time steps }\end{array}$ \\
\hline 80 & 1.6 & 0.721 & 40 & 1.55 & $\begin{array}{c}3237 \mathrm{~s} \\
60000 \text { time steps }\end{array}$ \\
\hline 80 & 1.6 & 0.721 & 50 & 1.55 & $\begin{array}{c}3850 \mathrm{~s} \\
60000 \text { time steps }\end{array}$ \\
\hline 80 & 1.6 & 0.721 & 60 & 1.55 & $\begin{array}{c}4445 \mathrm{~s} \\
60000 \text { time steps }\end{array}$ \\
\hline
\end{tabular}

Table C.6 Simulations with four layer lead in and lead out waveguide sections.

\begin{tabular}{|c|c|c|c|c|c|c|}
\hline $\begin{array}{c}\text { Resolution } \\
(\text { points/ } \\
\mu \mathrm{m})\end{array}$ & $\begin{array}{c}\text { Overlay } \\
\text { refractive } \\
\text { index }\end{array}$ & $\begin{array}{c}\text { Overlay } \\
\text { thickness } \\
(\mu \mathrm{m})\end{array}$ & $\begin{array}{c}\text { Waveguide } \\
\text { thickness } \\
(\mu \mathrm{m})\end{array}$ & $\begin{array}{c}\text { Overlay } \\
\text { length } \\
(\mu \mathrm{m})\end{array}$ & $\begin{array}{c}\text { Bragg } \\
\text { design } \\
\text { wavelength } \\
(\mu \mathrm{m})\end{array}$ & $\begin{array}{c}\text { Duration of } \\
\text { simulation }\end{array}$ \\
\hline 80 & 1.6 & 0.721 & 1.000 & 40 & 1.55 & $\begin{array}{c}3324 \mathrm{~s} \\
60000 \mathrm{steps}\end{array}$ \\
\hline 80 & 1.6 & 0.72 & 1.00 & 60 & 1.55 & $\begin{array}{c}4439 \mathrm{~s} \\
60000 \mathrm{steps}\end{array}$ \\
\hline 80 & 1.6 & 0.48 & 1.24 & 60 & 1.55 & $\begin{array}{c}4278 \mathrm{~s} \\
60000 \text { steps }\end{array}$ \\
\hline 80 & 1.6 & 0.24 & 1.48 & 60 & 1.55 & $\begin{array}{c}4613 \mathrm{~s} \\
60000 \text { steps }\end{array}$ \\
\hline 40 & 1.6 & 0.24 & 1.48 & 120 & 1.55 & $\begin{array}{c}2373 \mathrm{~s} \\
60000 \mathrm{steps}\end{array}$ \\
\hline
\end{tabular}




\begin{tabular}{|c|c|c|c|c|c|c|}
\hline 80 & 1.6 & 0.24 & 1.48 & 120 & 1.55 & $\begin{array}{c}7554 \mathrm{~s} \\
60000 \text { steps }\end{array}$ \\
\hline 40 & 1.6 & 0.24 & 1.48 & 180 & 1.55 & $\begin{array}{c}3390 \mathrm{~s} \\
60000 \text { steps }\end{array}$ \\
\hline 40 & 1.6 & 0.24 & 1.48 & 180 & 1.55 & $\begin{array}{c}4629 s \\
90000 \text { steps }\end{array}$ \\
\hline 40 & 1.6 & 0.24 & 1.48 & 180 & 1.55 & $\begin{array}{c}3047 \mathrm{~s} \\
60000 \text { steps } \\
\end{array}$ \\
\hline 40 & 1.6 & 0.24 & 1.48 & 180 & 1.55 & $\begin{array}{c}2733 \mathrm{~s} \\
60000 \text { steps }\end{array}$ \\
\hline 40 & 1.6 & 0.12 & 1.00 & 180 & 1.55 & $\begin{array}{c}3214 \mathrm{~s} \\
60000 \text { steps }\end{array}$ \\
\hline 40 & 1.6 & 0.06 & 1.00 & 180 & 1.55 & $\begin{array}{c}2922 \mathrm{~s} \\
60000 \text { steps }\end{array}$ \\
\hline 40 & 1.6 & 0.03 & 1.00 & 180 & 1.55 & $\begin{array}{c}2952 \mathrm{~s} \\
60000 \text { steps } \\
\end{array}$ \\
\hline 40 & 1.6 & 0.03 & 0.97 & 180 & 1.55 & $\begin{array}{c}2981 \mathrm{~s} \\
60000 \text { steps }\end{array}$ \\
\hline 40 & 1.6 & 0.06 & 0.94 & 180 & 1.55 & $\begin{array}{c}121 \mathrm{~s} \\
60000 \text { steps }\end{array}$ \\
\hline 40 & 1.6 & 0.12 & 0.88 & 180 & 1.55 & $\begin{array}{c}2879 \mathrm{~s} \\
60000 \text { steps }\end{array}$ \\
\hline 40 & 1.6 & 0.24 & 0.76 & 180 & 1.55 & $\begin{array}{c}2863 \mathrm{~s} \\
60000 \text { steps } \\
\end{array}$ \\
\hline 40 & 1.6 & 0.03 & 0.97 & 180 & 1.55 & $\begin{array}{c}2977 \mathrm{~s} \\
60000 \text { steps }\end{array}$ \\
\hline 40 & 1.6 & 0.03 & 0.97 & 180 & 1.55 & $\begin{array}{c}3097 \mathrm{~s} \\
60000 \text { steps }\end{array}$ \\
\hline 40 & 1.6 & 0.03 & 0.97 & 180 & 1.55 & $\begin{array}{c}2971 \mathrm{~s} \\
60000 \text { steps }\end{array}$ \\
\hline 40 & 1.6 & 0.03 & 0.97 & 180 & 1.55 & $\begin{array}{c}3005 \mathrm{~s} \\
60000 \text { steps }\end{array}$ \\
\hline 40 & 1.6 & 0.03 & 0.97 & 180 & 1.55 & $\begin{array}{c}4499 \mathrm{~s} \\
60000 \text { steps }\end{array}$ \\
\hline 40 & 1.6 & 0.03 & 0.97 & 180 & 1.55 & 60000 steps \\
\hline 80 & 1.6 & 0.03 & 0.97 & 180 & 1.55 & $\begin{array}{c}15871 \mathrm{~s}=-4.5 \mathrm{~h} \\
90000 \text { steps }\end{array}$ \\
\hline 80 & 1.6 & 0.03 & 0.97 & 180 & 1.55 & $\begin{array}{c}20866 s=\sim 5.8 h \\
120000 \text { steps }\end{array}$ \\
\hline 160 & 3.6 & 0.032 & 1.00 & 20 & 1.55 & $\begin{array}{c}7872 s=-2.2 h \\
60000 \text { steps }\end{array}$ \\
\hline 160 & 3.6 & 0.032 & 1.10 & 20 & 1.55 & $\begin{array}{c}7278 \mathrm{~s} \\
60000 \text { steps }\end{array}$ \\
\hline 160 & 3.6 & 0.032 & 0.90 & 20 & 1.55 & $6949 \mathrm{~s}$ \\
\hline
\end{tabular}




\begin{tabular}{|c|c|c|c|c|c|c|}
\hline 160 & 3.48 & 0.0347 & 1.00 & 20 & 1.55 & $\begin{array}{c}60000 \text { steps } \\
7334 \mathrm{~s} \\
60000 \text { steps }\end{array}$ \\
\hline 160 & 3.4 & 0.025 & 1.00 & 20 & 1.55 & $\begin{array}{c}20266 \mathrm{~s} \\
60000 \text { steps }\end{array}$ \\
\hline 160 & 3.4 & 0.028 & 1.00 & 20 & 1.55 & 60000 steps \\
\hline
\end{tabular}

\section{C.2 Parametric analysis simulations}

Simulations for the parametric analysis began as listed in table C.7. During this set of simulation convergence continued to be confirmed.

Table C.7 Simulations probing convergence of simulation results and structure parameters.

\begin{tabular}{|c|c|c|c|c|c|}
\hline $\begin{array}{l}\text { Resolution } \\
\text { (points/ } \mu \mathrm{m} \text { ) }\end{array}$ & $\begin{array}{l}\text { Overlay } \\
\text { refractive } \\
\text { index }\end{array}$ & $\begin{array}{c}\text { Overlay } \\
\text { thickness } \\
(\mu \mathrm{m})\end{array}$ & $\begin{array}{c}\text { Overlay } \\
\text { length } \\
(\mu \mathrm{m})\end{array}$ & $\begin{array}{l}\text { Bragg design } \\
\text { wavelength } \\
(\mu \mathrm{m})\end{array}$ & $\begin{array}{l}\text { Duration of } \\
\text { simulation }\end{array}$ \\
\hline 160 & 3.6 & 0.015 & 10 & 1.55 & $134440 \mathrm{~s}$ \\
\hline 160 & 3.6 & 0.015 & 30 & 1.55 & $638568 s$ \\
\hline 160 & 3.1 & 0.015 & 18.5 & 1.55 & $144385 \mathrm{~s}$ \\
\hline 160 & 2.6 & 0.015 & 18.5 & 1.55 & $161608 \mathrm{~s}$ \\
\hline 160 & 1.6 & 0.015 & 18.5 & 1.55 & $144680 \mathrm{~s}$ \\
\hline 40 & 3.6 & 0.025 & 18.5 & 1.55 & $791 \mathrm{~s}$ \\
\hline 80 & 3.6 & 0.025 & 18.5 & 1.55 & $16686 \mathrm{~s}$ \\
\hline 120 & 3.6 & 0.025 & 18.5 & 1.55 & $85490 \mathrm{~s}$ \\
\hline 150 & 3.6 & 0.025 & 18.5 & 1.55 & $93386 \mathrm{~s}$ \\
\hline 160 & 3.6 & 0.025 & 18.5 & 1.55 & $135214 \mathrm{~s}=\sim 38 \mathrm{~h}$ \\
\hline 40 & 3.6 & 0.026 & 18.5 & 1.55 & $7702 \mathrm{~s}$ \\
\hline 80 & 3.6 & 0.026 & 18.5 & 1.55 & $14373 \mathrm{~s}$ \\
\hline 120 & 3.6 & 0.026 & 18.5 & 1.55 & $46736 \mathrm{~s}$ \\
\hline 150 & 3.6 & 0.026 & 18.5 & 1.55 & $96031 \mathrm{~s}=\sim 27 \mathrm{~h}$ \\
\hline 160 & 3.6 & 0.026 & 18.5 & 1.55 & $128878 \mathrm{~s}$ \\
\hline 160 & 3.6 & 0.025 & 18.5 & 1.4091 & $288399 \mathrm{~s}$ \\
\hline 160 & 3.6 & 0.025 & 18.5 & 1.4762 & $142996 \mathrm{~s}$ \\
\hline 160 & 3.6 & 0.025 & 18.5 & 1.55 & $135214 \mathrm{~s}$ \\
\hline 160 & 3.6 & 0.025 & 18.5 & 1.6316 & $134188 \mathrm{~s}$ \\
\hline 160 & 3.6 & 0.025 & 18.5 & 1.7222 & $140542 \mathrm{~s}$ \\
\hline 160 & 3.6 & 0.032 & 10 & 1.55 & $117071 \mathrm{~s}=-33 \mathrm{~h}$ \\
\hline 160 & 3.6 & 0.032 & 20 & 1.55 & $148449 \mathrm{~s}$ \\
\hline 160 & 3.6 & 0.032 & 30 & 1.55 & $664389 \mathrm{~s}=\sim 185 \mathrm{~h}$ \\
\hline 160 & 3.6 & 0.032 & 18.5 & 1.55 & $133363 \mathrm{~s}$ \\
\hline 160 & 3.6 & 0.030 & 18.5 & 1.55 & $129532 \mathrm{~s}$ \\
\hline 160 & 3.6 & 0.026 & 18.5 & 1.55 & $128878 \mathrm{~s}$ \\
\hline
\end{tabular}




\begin{tabular}{|c|c|c|c|c|c|}
\hline 160 & 3.6 & 0.025 & 18.5 & 1.55 & $135214 \mathrm{~s}$ \\
\hline 160 & 3.6 & 0.020 & 18.5 & 1.55 & $137884 \mathrm{~s}$ \\
\hline 160 & 3.6 & 0.015 & 18.5 & 1.55 & $143261 \mathrm{~s}$ \\
\hline 160 & 3.6 & 0.025 & 18.5 & 1.55 & $135214 \mathrm{~s}$ \\
\hline 160 & 3.1 & 0.025 & 18.5 & 1.55 & $142374 \mathrm{~s}$ \\
\hline 160 & 2.6 & 0.025 & 18.5 & 1.55 & $149050 \mathrm{~s}$ \\
\hline 160 & 2.1 & 0.025 & 18.5 & 1.55 & $157160 \mathrm{~s}$ \\
\hline 160 & 1.6 & 0.025 & 18.5 & 1.55 & $139210 \mathrm{~s}$ \\
\hline 160 & 3.6 & 0.025 & 10 & 1.55 & $127220 \mathrm{~s}=-35 \mathrm{~h}$ \\
\hline 160 & 3.6 & 0.025 & 20 & 1.55 & $346488 s=-96 h$ \\
\hline 160 & 3.6 & 0.025 & 30 & 1.55 & $\begin{array}{c}273658 \mathrm{~s}=\sim 76 \mathrm{~h} \\
255139 \text { time steps }\end{array}$ \\
\hline 160 & 3.6 & 0.025 & 40 & 1.55 & $420971 \mathrm{~s}=\sim 117 \mathrm{~h}$ \\
\hline 160 & 3.6 & 0.025 & 50 & 1.55 & $\begin{array}{c}710912 \mathrm{~s}=198 \mathrm{~h} \\
438392 \text { time steps }\end{array}$ \\
\hline 160 & 3.6 & 0.032 & 10 & 1.55 & $62197 \mathrm{~s}=\sim 17.3 \mathrm{~h}$ \\
\hline 160 & 3.6 & 0.032 & 10 & 1.55 & $\begin{array}{c}2658 \mathrm{~s}=\sim 0.75 \mathrm{~h} \\
25575 \text { time steps }\end{array}$ \\
\hline 160 & 3.6 & 0.032 & 10 & 1.55 & $\begin{array}{c}1753 \mathrm{~s} \\
16962 \text { time steps }\end{array}$ \\
\hline 160 & 3.6 & 0.032 & 10 & 1.55 & $\begin{array}{c}1480 \mathrm{~s} \\
16972 \text { time steps }\end{array}$ \\
\hline 160 & 3.6 & 0.032 & 10 & 1.55 & $2175 \mathrm{~s}$ \\
\hline 160 & 3.6 & 0.032 & 10 & 1.55 & $2535 \mathrm{~s}$ \\
\hline 160 & 3.6 & 0.032 & 30 & 1.55 & $5095 \mathrm{~s}=\sim 1.5 \mathrm{~h}$ \\
\hline 160 & 3.6 & 0.032 & 10 & 1.55 & $26918 \mathrm{~s}=\sim 7.5 \mathrm{~h}$ \\
\hline 160 & 3.6 & 0.032 & 10 & 1.55 & $56913 s=\sim 16 h$ \\
\hline 160 & 3.6 & 0.032 & 10 & 1.55 & $\begin{array}{c}2869 \mathrm{~s} \\
32000 \text { time steps }\end{array}$ \\
\hline 160 & 3.6 & 0.032 & 10 & 1.55 & $\begin{array}{c}2923 \mathrm{~s} \\
32000 \text { time steps }\end{array}$ \\
\hline 160 & 3.6 & 0.032 & 10 & 1.9375 & $\begin{array}{c}2909 \mathrm{~s} \\
32000 \text { time steps }\end{array}$ \\
\hline 160 & 3.6 & 0.032 & 10 & 1.55 & $\begin{array}{c}26918 \mathrm{~s}=\sim 7.5 \mathrm{~h} \\
240000 \text { time steps }\end{array}$ \\
\hline 160 & 3.6 & 0.032 & 20 & 1.55 & $\begin{array}{c}6280 \mathrm{~s}=\sim 1.75 \mathrm{~h} \\
40000 \text { time steps }\end{array}$ \\
\hline 160 & 3.6 & 0.032 & 20 & 1.55 & $\begin{array}{c}5902 \mathrm{~s} \\
50000 \text { time steps }\end{array}$ \\
\hline 160 & 3.6 & 0.032 & 20 & 1.55 & $\begin{array}{c}11969 \mathrm{~s}=\sim 3.3 \mathrm{~h} \\
100000 \text { time steps }\end{array}$ \\
\hline 160 & 3.6 & 0.032 & 30 & 1.55 & $\begin{array}{c}19556 \mathrm{~s}=\sim 5.5 \mathrm{~h} \\
100000 \text { time steps }\end{array}$ \\
\hline 160 & 3.6 & 0.032 & 30 & 1.55 & $18230 \mathrm{~s}=\sim 5.1 \mathrm{~h}$ \\
\hline
\end{tabular}




\begin{tabular}{|c|c|c|c|c|c|}
\hline & & & & & 120000 time steps \\
\hline 160 & 3.6 & 0.032 & 40 & 1.55 & $\begin{array}{c}2265185 \mathrm{~s}=\sim 26.2 \mathrm{~d} \\
1589000 \text { time steps }\end{array}$ \\
\hline 160 & 3.6 & 0.032 & 20 & 1.55 & $\begin{array}{c}22172 \mathrm{~s}=\sim 6.2 \mathrm{~h} \\
60000 \text { time steps }\end{array}$ \\
\hline 160 & 3.6 & 0.032 & 20 & 1.55 & $\begin{array}{c}6721 \mathrm{~s}=\sim 1.9 \mathrm{~h} \\
60000 \text { time steps }\end{array}$ \\
\hline 160 & 3.6 & 0.020 & 10 & 1.4091 & 30000 time steps \\
\hline 160 & 3.6 & 0.026 & 10 & 1.4091 & 30000 time steps \\
\hline 160 & 3.6 & 0.032 & 10 & 1.4091 & 30000 time steps \\
\hline 160 & 3.6 & 0.038 & 10 & 1.4091 & 30000 time steps \\
\hline 160 & 3.6 & 0.020 & 10 & 1.5500 & 30000 time steps \\
\hline 160 & 3.6 & 0.026 & 10 & 1.5500 & 30000 time steps \\
\hline 160 & 3.6 & 0.032 & 10 & 1.5500 & 30000 time steps \\
\hline 160 & 3.6 & 0.038 & 10 & 1.5500 & 30000 time steps \\
\hline 160 & 3.6 & 0.020 & 10 & 1.7222 & 30000 time steps \\
\hline 160 & 3.6 & 0.026 & 10 & 1.7222 & 30000 time steps \\
\hline 160 & 3.6 & 0.032 & 10 & 1.7222 & 30000 time steps \\
\hline 160 & 3.6 & 0.038 & 10 & 1.7222 & 30000 time steps \\
\hline 160 & 3.6 & 0.020 & 10 & 1.9375 & 30000 time steps \\
\hline 160 & 3.6 & 0.026 & 10 & 1.9375 & 30000 time steps \\
\hline 160 & 3.6 & 0.032 & 10 & 1.9375 & 30000 time steps \\
\hline 160 & 3.6 & 0.038 & 10 & 1.9375 & 30000 time steps \\
\hline 160 & 3.6 & 0.020 & 20 & 1.4091 & 60000 time steps \\
\hline 160 & 3.6 & 0.026 & 20 & 1.4091 & 60000 time steps \\
\hline 160 & 3.6 & 0.032 & 20 & 1.4091 & 60000 time steps \\
\hline 160 & 3.6 & 0.038 & 20 & 1.4091 & 60000 time steps \\
\hline 160 & 3.6 & 0.020 & 20 & 1.5500 & 60000 time steps \\
\hline 160 & 3.6 & 0.026 & 20 & 1.5500 & 60000 time steps \\
\hline 160 & 3.6 & 0.032 & 20 & 1.5500 & 60000 time steps \\
\hline 160 & 3.6 & 0.038 & 20 & 1.5500 & 60000 time steps \\
\hline 160 & 3.6 & 0.020 & 20 & 1.7222 & 60000 time steps \\
\hline 160 & 3.6 & 0.026 & 20 & 1.7222 & 60000 time steps \\
\hline 160 & 3.6 & 0.032 & 20 & 1.7222 & 60000 time steps \\
\hline 160 & 3.6 & 0.038 & 20 & 1.7222 & 60000 time steps \\
\hline 160 & 3.6 & 0.020 & 20 & 1.9375 & 60000 time steps \\
\hline 160 & 3.6 & 0.026 & 20 & 1.9375 & 60000 time steps \\
\hline 160 & 3.6 & 0.032 & 20 & 1.9375 & 60000 time steps \\
\hline & 3.6 & 0.038 & 20 & 1.9375 & 60000 time steps \\
\hline
\end{tabular}




\section{C.3 Sensor structure simulations}

Initially sensor structure simulations were run for varying saline concentrations and sensor interaction lengths. The simulations listed in table C.8 began to investigate the sensor structure with the decided upon length and with glycerol in mind.

Table C.8 Sensor structure simulations to determine the response for mixtures of water and glycerol.

\begin{tabular}{|c|c|c|c|c|c|}
\hline $\begin{array}{c}\text { Resolution } \\
(\text { points } / \mu \mathrm{m})\end{array}$ & $\begin{array}{c}\text { Superstrate } \\
\text { refractive } \\
\text { index }\end{array}$ & $\begin{array}{c}\text { Overlay } \\
\text { thickness } \\
(\mu \mathrm{m})\end{array}$ & $\begin{array}{c}\text { Overlay } \\
\text { length } \\
(\mu \mathrm{m})\end{array}$ & $\begin{array}{c}\text { Bragg design } \\
\text { wavelength } \\
(\mu \mathrm{m})\end{array}$ & $\begin{array}{c}\text { Duration of } \\
\text { simulation }\end{array}$ \\
\hline 160 & 1.3300 & 0.020 & 20 & 1.55 & 60000 time steps \\
\hline 160 & $1.3541^{*}$ & 0.020 & 20 & 1.55 & 60000 time steps \\
\hline 160 & $1.3782^{*}$ & 0.020 & 20 & 1.55 & 60000 time steps \\
\hline 160 & $1.4023^{*}$ & 0.020 & 20 & 1.55 & 60000 time steps \\
\hline 160 & $1.4264^{*}$ & 0.020 & 20 & 1.55 & 60000 time steps \\
\hline 160 & $1.4505^{*}$ & 0.020 & 20 & 1.55 & 60000 time steps \\
\hline 160 & 1.4746 & 0.020 & 20 & 1.55 & 60000 time steps \\
\hline
\end{tabular}

*For these simulations the summary of results charts follow the table as examples. 

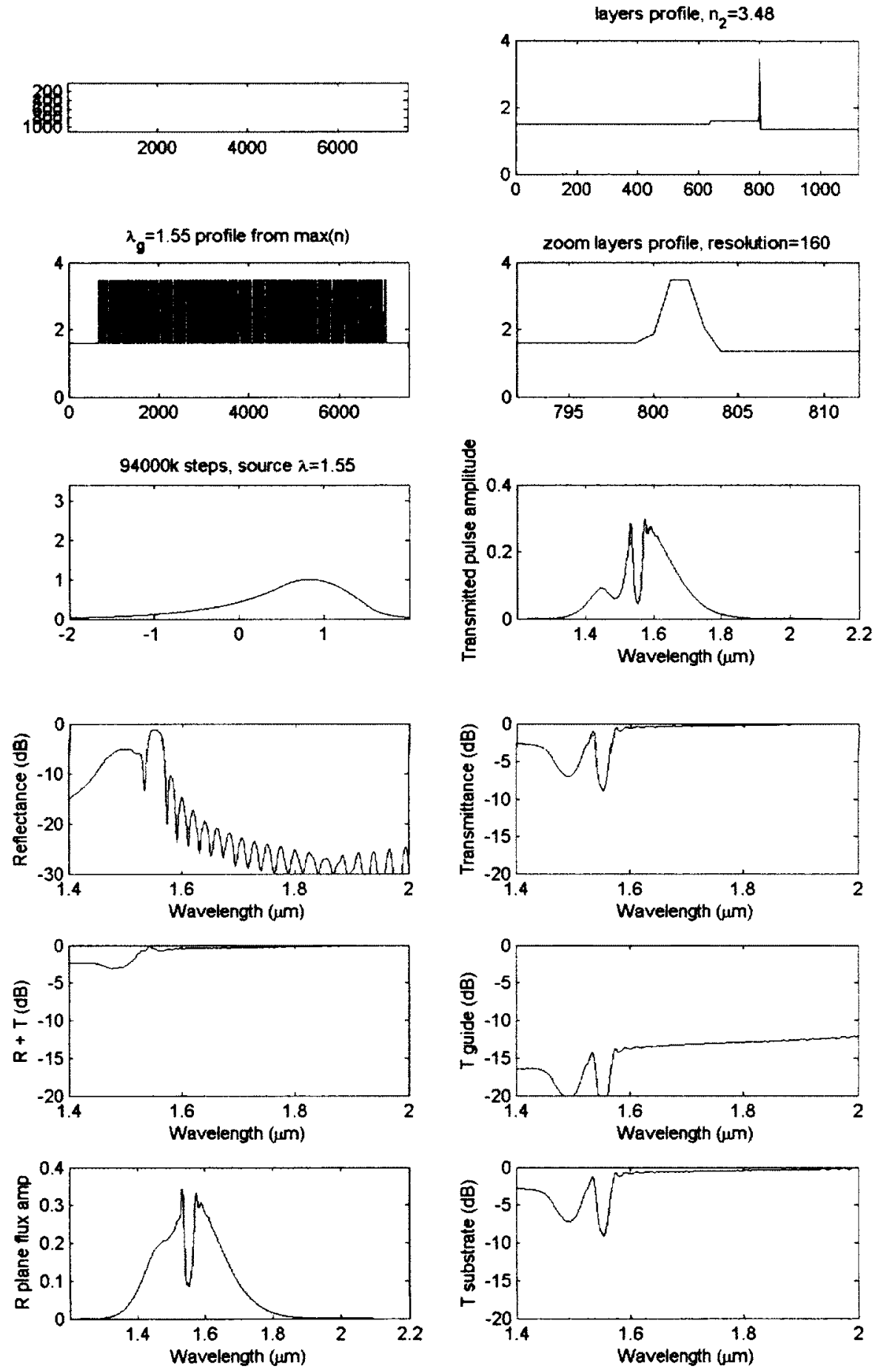

Figure C.1 Summary of results chart for a sensor structure superstrate refractive index of $\mathbf{1 . 3 5 + 1}$. 

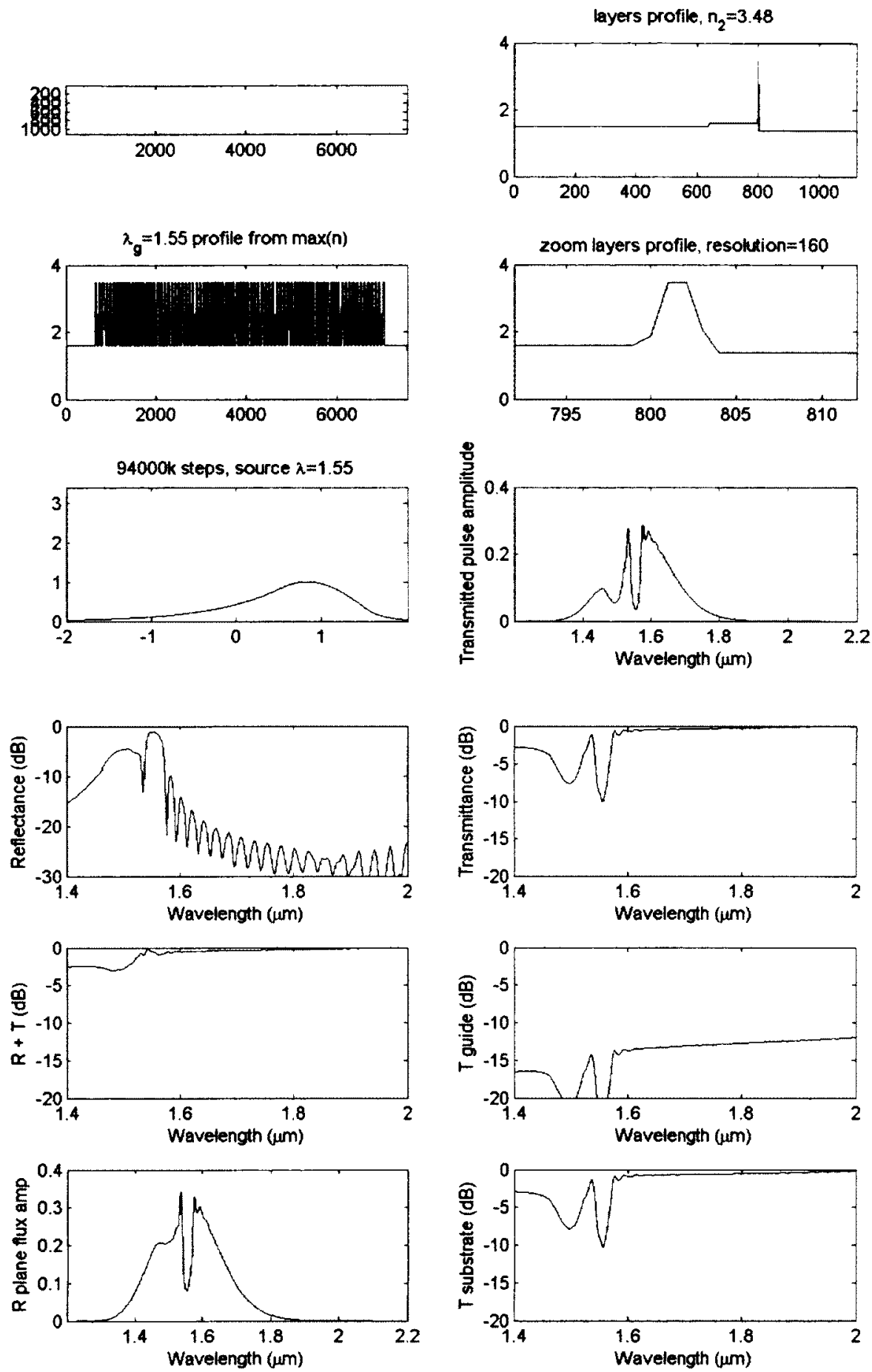

Figure C.2 Summary of results chart for a sensor structure superstrate refractive index of $\mathbf{1 . 3 7 9 2}$.

112 

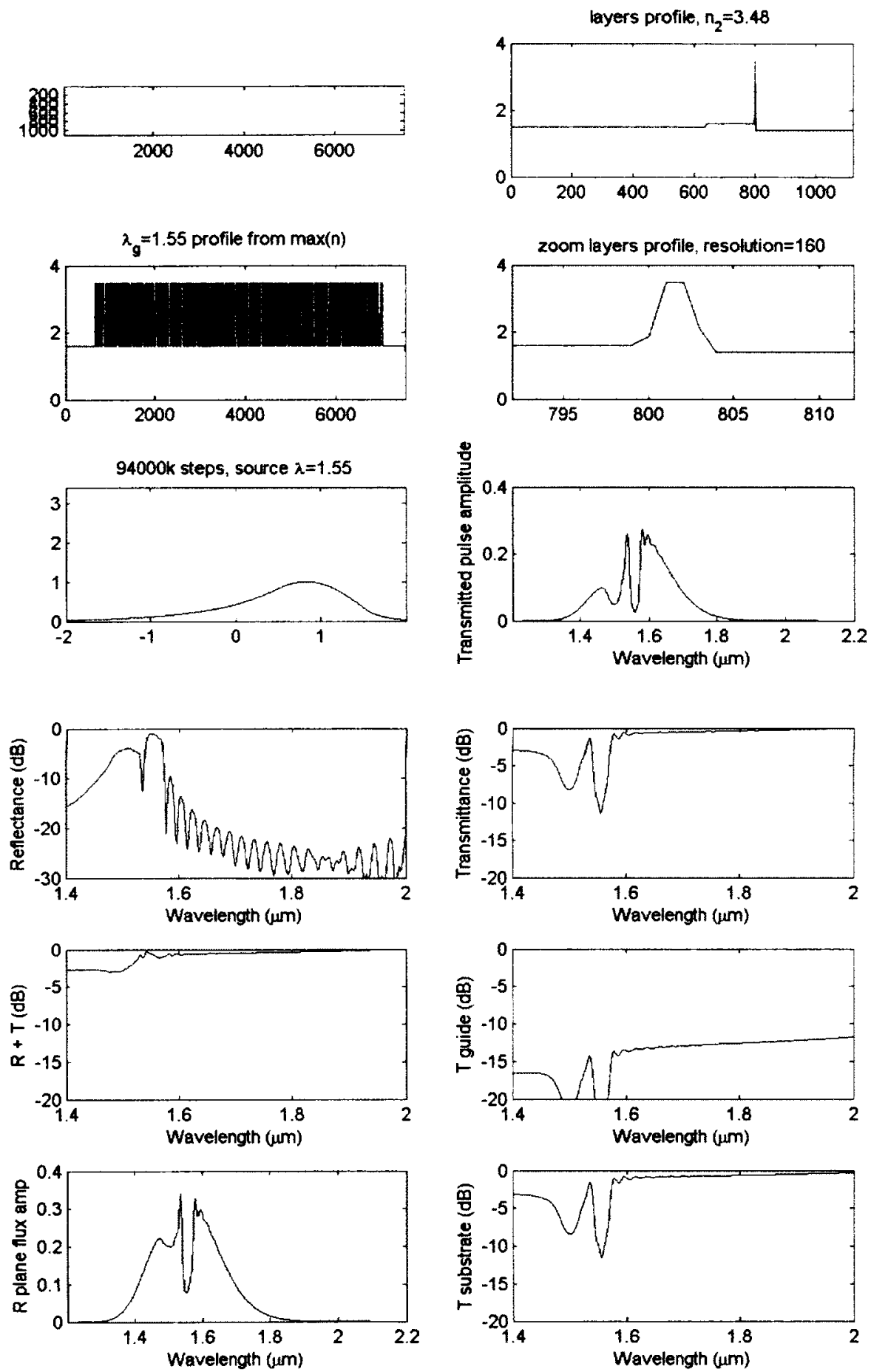

Figure C.3 Summary of results chart for a sensor structure superstrate refractive index of 1.4023. 

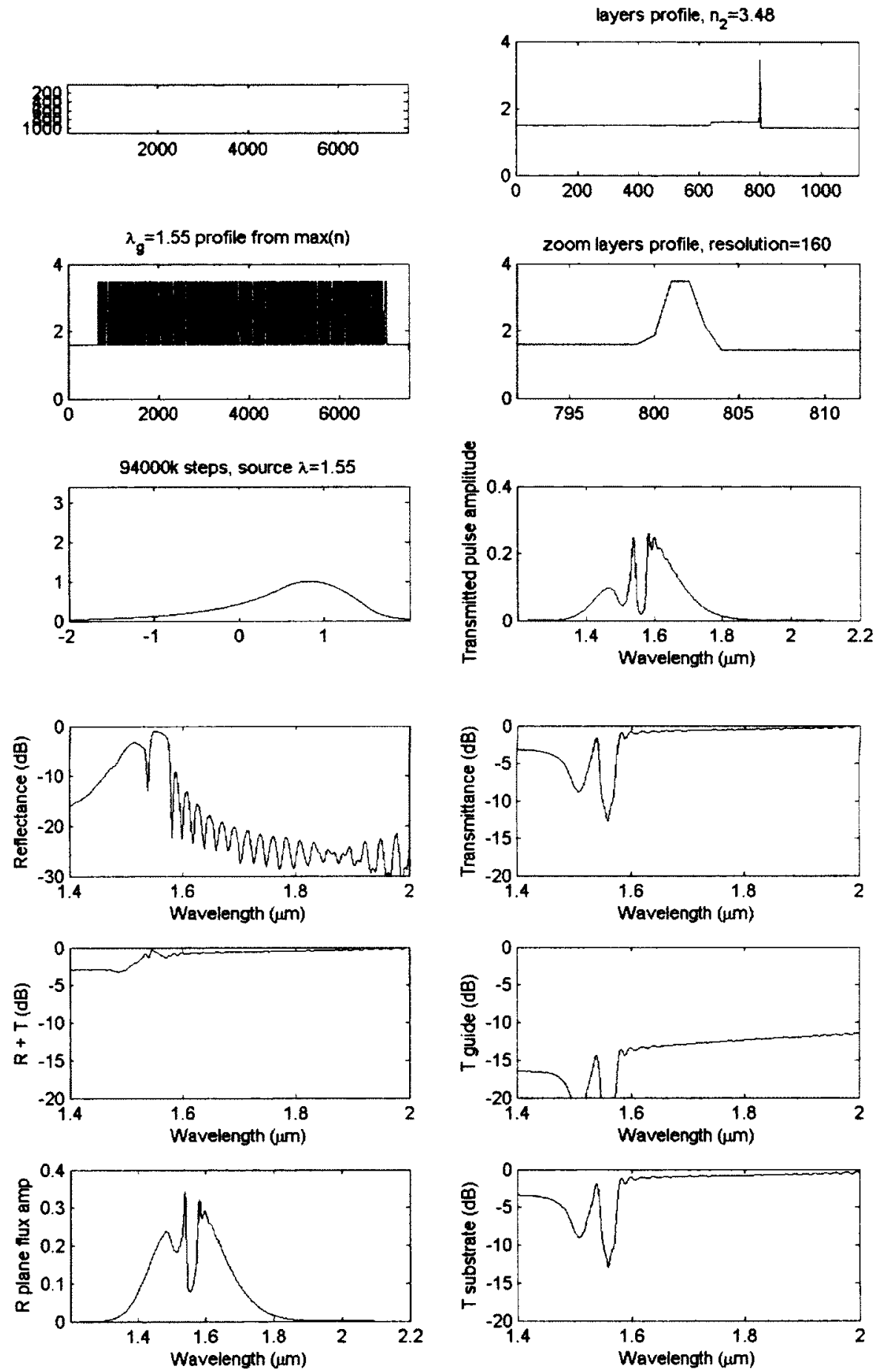

Figure C.4 Summary of results chart for a sensor structure superstrate refractive index of $\mathbf{1 . 4 2 6 4}$. 

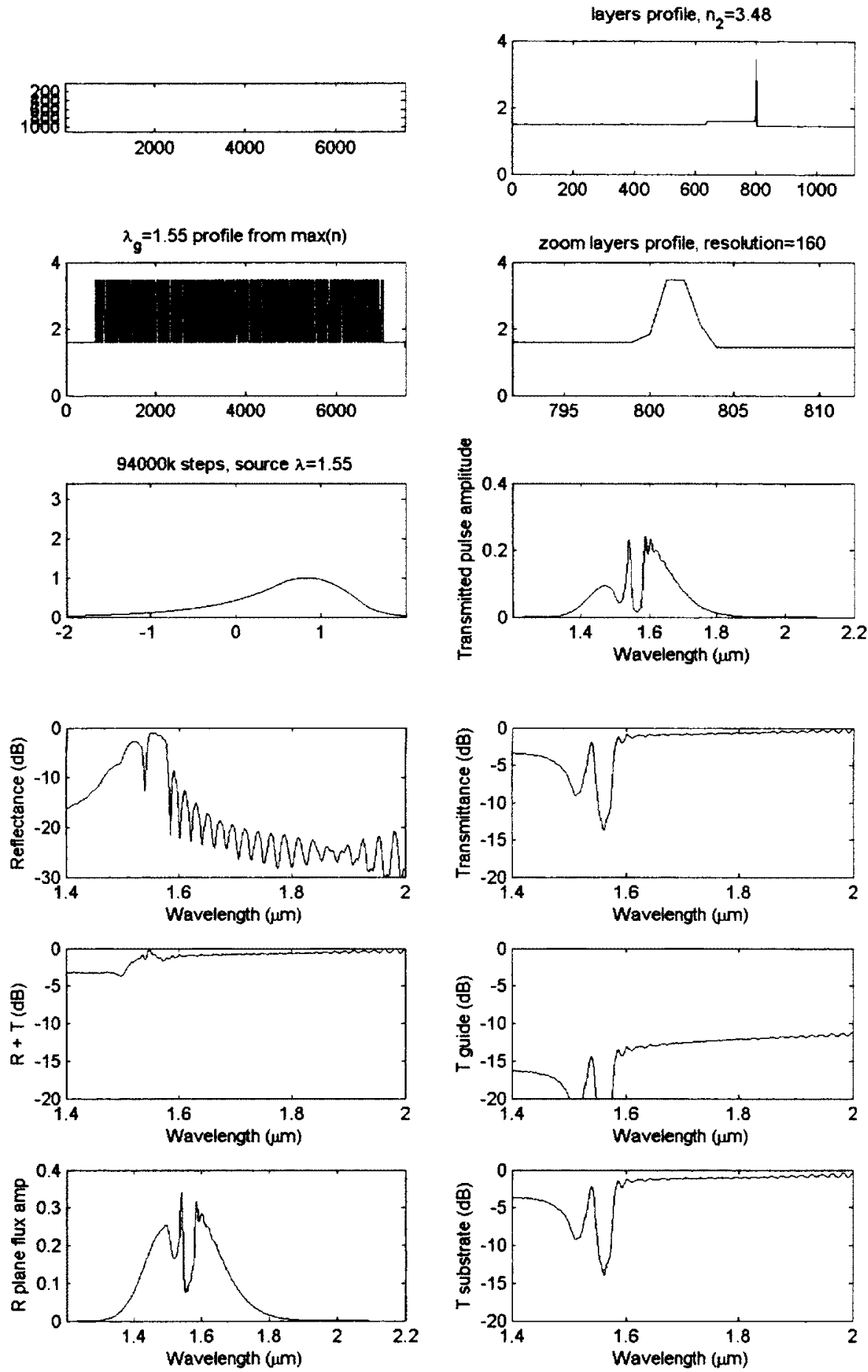

Figure C.5 Summary of results chart for a sensor structure superstrate refractive index of 1.4505.

115 


\section{C.4 3-D simulations}

Table C.9 lists the 3-D simulations which were run on several platforms as discussed in appendix B.

Table C.9 List of 3-D simulations run including the amount of RAM that simulation of the structure required and the duration of the execution of a corresponding simulation time step.

\begin{tabular}{|c|c|c|c|}
\hline Dimensions in $\mu \mathrm{m}$ & $\begin{array}{c}\text { Resolution } \\
\text { (points/ } \mu \mathrm{m})\end{array}$ & GB RAM & s/step \\
\hline $3 \times 3 \times 10$ & 10 & 0.821 & 0.03 \\
\hline $3 \times 3 \times 10$ & 20 & 1.204 & 0.1 \\
\hline $3 \times 3 \times 10$ & 30 & 2.179 & 0.3 \\
\hline $3 \times 3 \times 10$ & 40 & 3.922 & 0.6 \\
\hline $3 \times 3 \times 10$ & 50 & 6.737 & 1.3 \\
\hline $7 \times 7 \times 20$ & 40 & 20.562 & 4 \\
\hline $3 \times 3 \times 10$ & 160 & $\mathrm{n} / \mathrm{a}$ & $\begin{array}{c}11 \\
\text { plus } 1.5 \mathrm{~h} \text { to discretize } \\
\text { simulation domain }\end{array}$ \\
\hline
\end{tabular}

116 


\section{Appendix D Multimode and taper structures}

This appendix examines several structures of interest that were discovered alongside the main body of this work.[60,61] In the multimode discussion in section 3.2 , it is discovered that it is possible to have the effective index of a 3 layer mode and a 4 layer mode match. This results in the field profile of the fundamental mode of the 3 layer waveguide overlapping exactly for exceptional coupling in the substrate and waveguide regions with the field profile of the second mode when the overlay is added. The second novel feature was that the MMI behaviour of the 4 layer waveguide structure between two 3 layer waveguide sections provides layer specific coupling control. Expanding on these a third interesting feature is the way tapers can be used to couple to the overlay. Although in the previous finding, a 4 layer mode overlaps well with a three layer mode, the energy is still predominantly in the guiding layer. In order to couple energy into the overlay, it is possible to both taper the underlying waveguide and taper the overlay such that the mode is transitioned into the overlay. Figure D.1 shows the cross section of such a structure with the addition of the reverse process, allowing the mode to be coupled back into the waveguide.

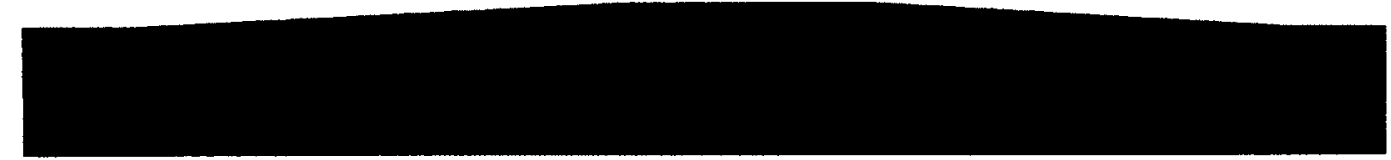

Figure D.1 Double taper layer configuration shown by shading in proportion to refractive index. In addition, the taper can take place from above such that the overlay is placed directly on the substrate akin to SOI and the guiding layer is deposited afterward. Figure D. 2 shows 
such a structure showing the optional tapering of the deposited guiding layer with a dashed line.

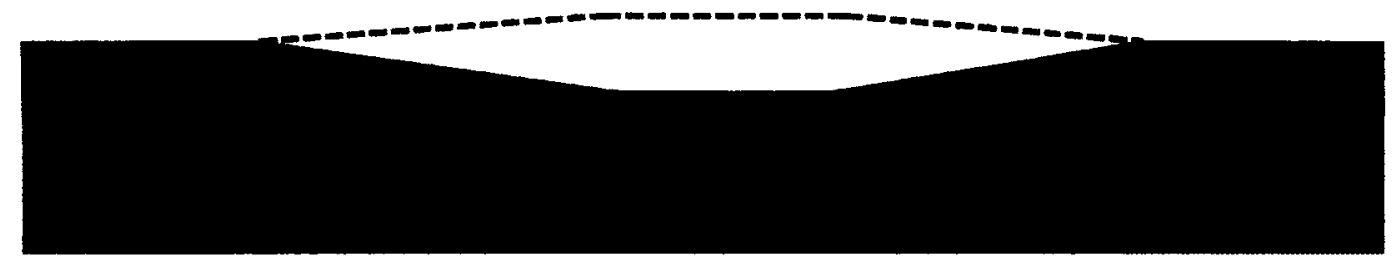

Figure D.2 Over-guide configuration in the SOI platform showing optional removal of a portion of the guiding layer material.

\section{D.1 Structure details for simulation}

The source polarization considered for these structures is TE with a wavelength ranging from $1.2 \mu \mathrm{m}$ to $2.0 \mu \mathrm{m}$. For the overlapping 3 and 4 layer modes with the same effective index, the waveguide is $2 \mu \mathrm{m}$ thick with an index of refraction of 1.60 and the overlay is $285 \mathrm{~nm}$ thick with an index of refraction of 3.48 . The superstrate is taken to have an index of refraction of 1.00 and the substrate is taken to have an index of refraction of 1.50. The field equations were analytically solved and for modal simulation, a $10 \mu \mathrm{m}$ three layer section is followed by a $100 \mu \mathrm{m}$ four layer region. For the MMI simulation, the 3 layer lead in is $5 \mu \mathrm{m}$ long and the 4 layer structure with the un-patterned overlay is $40 \mu \mathrm{m}$ long followed by a $5 \mu \mathrm{m}$ long 3 layer exit waveguide. The superstrate above the overlay is taken to have a nominal refractive index of 1.00 unless used as a sensor in which case it takes the value of the material to be sensed. The length of the four layer section is also modified for sensor applications. The structure is discretized on a square grid of 240 points per micron with a $1.5 \mu \mathrm{m}$ thick PML. 
The design of the coupling tapers uses a waveguide $1 \mu \mathrm{m}$ thick with no overlay which is transitioned by using a $50 \mu \mathrm{m}$ long tapered section to the full thickness of the high refractive index overlay of $250 \mathrm{~nm}$ and no extra waveguide. The modal overlap was evaluated every $0.1 \mu \mathrm{m}$ along the tapers. The taper structure with the guide deposited over the tapered SOI-like high refractive index layer is simulated for both the case with the tapered guide and the case with the guide at a consistent thickness.

\section{D.2 Simulation results}

For the multimode structure the modal approach, results in figure D.3 demonstrate the feature where the 3 layer mode overlaps the 4 layer mode identically in the substrate and waveguide for a wavelength of $1.78 \mu \mathrm{m}$. Also demonstrated is the self-imaging and periodic behaviour at the design wavelength of $1.55 \mu \mathrm{m}$.

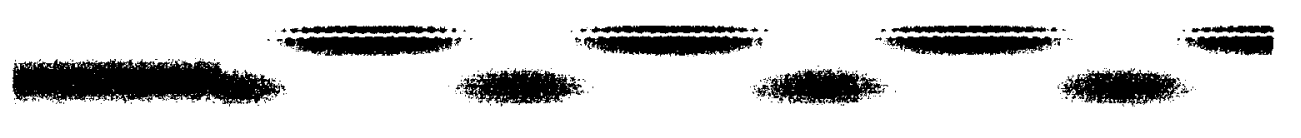

Figure D.3 Modal approach results for wavelengths of 1.55 and $1.78 \mu \mathrm{m}$ from top to bottom respectively.

For both the well overlapped case and the MMI case, the modal evolution is shown with the addition of the time varying component from the FDTD simulation as pictured in figure D.4. The FDTD simulation results also differ from the modal result appearance due to the shorter extent of both the overlay and the entire domain in the propagation direction. The scattering of the light is also apparent for the wavelength of $1.55 \mu \mathrm{m}$ due 119 
to the length of the overlay being selected at a point where coupling has taken place to the overlay and not a self-imaging length.

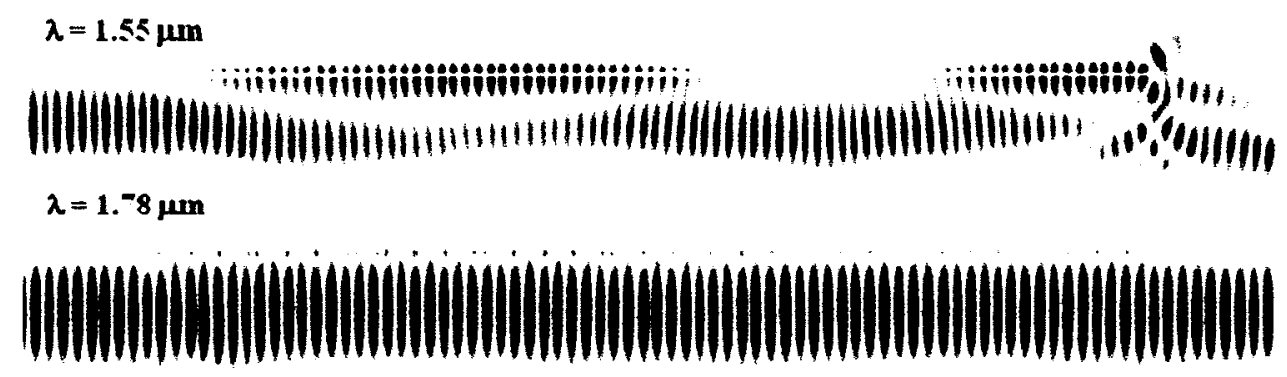

Figure D.4 FDTD results for wavelengths of 1.55 and $1.78 \mu \mathrm{m}$ from top to bottom respectively.

For the sensor application, a 4 layer section length of $32.4 \mu \mathrm{m}$ is simulated to demonstrate a reduction trend in the structure's output 3 layer power as the superstrate refractive index value increases in a range from 1.00 representing air to be able to sense low index gasses. In addition, this 4 layer section length provides increasing output 3 layer power for a second range starting from a refractive index of 1.315 representing pure water increasing towards refractive indices representative of saturated glucose solutions. As shown in figure D.5, a longer length can be used to provide higher sensitivity for smaller ranges. 


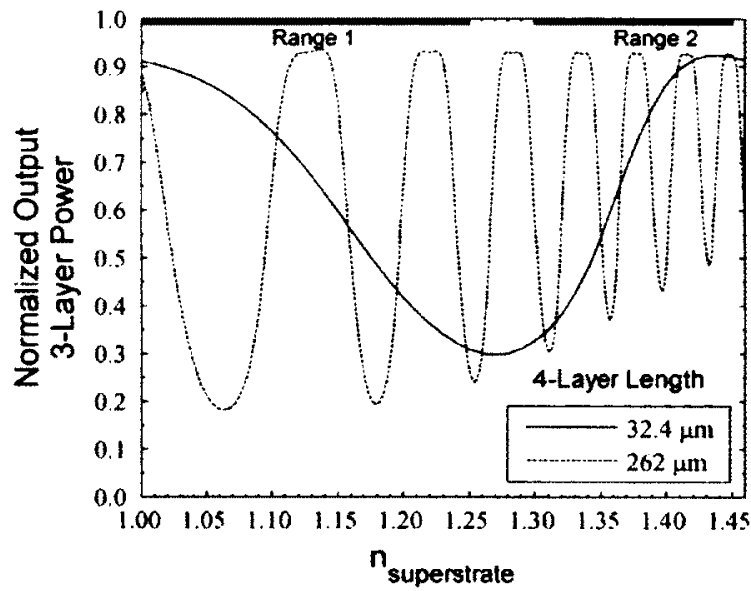

Figure D.5 Three layer output power versus 4 layer superstrate refractive index for lengths of 32.4 $\mu \mathrm{m}$ and $262 \mu \mathrm{m}$.

The modal approach is used to address coupling to high dielectric modes and the double taper is found to couple $98.2 \%$ of the input light into the high dielectric layer mode. The FDTD simulation result of this double taper configuration pictured in figure D.1 is shown in figure D.6.

Figure D.6 FDTD field distribution for double taper structure with the design wavelength of 1.55 $\mu \mathbf{m}$.

Using the time averaged pointing vector, the FDTD result is found to have coupled $96.2 \%$ of the input light into the high dielectric. Calculating the overlap integral of the modes of the two 3 layer structures provides the direct coupling field coefficient of 0.17 . When the high dielectric overlay is applied directly to the substrate and the guiding layer is deposited over it, simulations are again conducted using a modal approach as well as 121 
FDTD. The results are shown in figure D.7 for both including the removal and tapering of the deposited layer over the high dielectric layer as well as for the case with the constant thickness deposited waveguide.

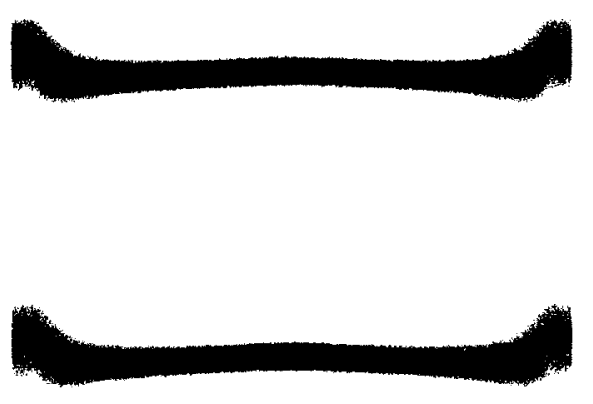

Figure D.7 Modal approach results on left and FDTD results on right for taper coupler with deposited waveguide also tapered and removed above dielectric (top images) and with constant thickness deposited waveguide (bottom images).

\section{D.3 Comparison and performance evaluation}

A brief foray has shown that multimode waveguides can provide alternate coupling scenarios and devices not possible in single mode configurations. The well overlapped 4 layer mode can be well coupled to; however, then the light continues to propagate primarily in a mode similar to the 3 layer mode without much energy in the overlay. However, at the correct wavelength, an MMI like behaviour is demonstrated with the energy transitioning back and forth from the guide to the overlay. When the appropriate length is chosen, this can provide device opportunities for filters, sensors, etc. The sensor results demonstrate a trade-off of sensitivity and specificity. A shorter 4 layer length not only has less sensor area but also provides distinguishable readings for a larger range of 122 
refractive indices while a longer 4 layer sensing region provides higher sensitivity albeit with a smaller range of distinguishable readings.

With some fabrication effort, taper couplers can provide many times more efficient coupling to high dielectric waveguides from glass than direct coupling albeit consuming platform real estate. The modal method and FDTD simulation results are in close agreement. The investigation of depositing the waveguide over top of the high refractive index dielectric layer provides a useful method of coupling from glass mode dimensions yet allowing the benefits of high refractive index devices typical of the SOI platform without substantial power losses. It is interesting that the removal and tapering of the deposited guide over the high dielectric region does not significantly change the simulation results. This suggests that this step is not a necessary added complexity since, although the structure becomes multimode, only the fundamental mode is coupled to provided that the conditions are not changed significantly from the simulation. It would be important to consider the design conditions when considering longer devices with increased opportunity for scattering from surface roughness. 


\section{References}

[1] M. Kawachi, "Recent progress in silica-based planar lightwave circuits on silicon," IEE Proceedings: Optoelectronics, vol. 143, pp. 257-262, 1996.

[2] J. Broquin, "Glass integrated optics: State of the art and position toward other technologies," in Integrated Optics: Devices, Materials, and Technologies XI, 2007, pp. 647507-1.

[3] R. T. Chen, "Hybrid and monolithic integration of planar lightwave circuits (PLCs)," in Integrated Optics: Devices, Materials, and Technologies XII, 2008, pp. 689611-1.

[4] J. D. Joannopoulos, S. G. Johnson, J. N. Winn and R. D. Meade, Photonic Crystals: Molding the Flow of Light (Second Edition). Princeton University Press, 2008.

[5] R. A. Bellman, G. Bourdon, G. Alibert, A. Beguin, E. Guiot, L. B. Simpson, P. Lehuede, L. Guiziou and E. LeGuen, "Ultralow loss high delta silica germania planar waveguides," J. Electrochem. Soc., vol. 151, pp. G541-G547, 2004.

[6] P. J. Bock, P. Cheben, J. H. Schmid, J. Lapointe, A. Delage, S. Janz, G. C. Aers, D. $\mathrm{Xu}, \mathrm{A}$. Densmore and T. J. Hall, "Subwavelength grating periodic structures in siliconon-insulator: A new type of microphotonic waveguide," Optics Express, vol. 18, pp. 20251-20262, 2010.

[7] A. A. Bettiol, E. J. Teo, S. Prashant, X. Boqian and M. B. H. Breese, "Fabrication of porous silicon channel waveguides with multilayer bragg cladding," in Silicon Photonics $V$, January 24, 2010 - January 27, 2010, pp. The Society of Photo-Optical Instrumentation Engineers (SPIE).

[8] G. B. Hocker, "Strip-loaded diffused optical waveguides," IEEE J. Quant. Electron., vol. QE12, pp. 232-6, 04, 1976.

[9] K. E. Medri and R. C. Gauthier, "Patterned overlays: thin silicon layer applied to glass waveguides," Proc. of SPIE, vol. 7943, pp. 0L-1-0L-12, 2011.

[10] J. Kim, K. A. Winick, C. Florea and M. McCoy, "Design and fabrication of low-loss hydrogenated amorphous silicon overlay DBR for glass waveguide devices," IEEE Journal on Selected Topics in Quantum Electronics, vol. 8, pp. 1307-1315, 2002.

[11] K. E. Medri and R. C. Gauthier, "Design of a Silicon Overlay Glass Waveguide Sensor," OPJ, vol. 1, December 2011, 2011.

[12] A. Yimit, A. G. Rossberg, T. Amemiya and K. Itoh, "Thin film composite optical waveguides for sensor applications: A review," Talanta, vol. 65, pp. 1102-1109, 2005.

[13] T. M. Libish, M. C. Bobby, J. Linesh, P. Biswas, S. Bandyopadhyay, K. Dasgupta and $P$. Radhakrishnan, "The effect of grating period on refractive index sensitivity of long period gratings written in hydrogen loaded SMF-28 fiber," Journal of Optoelectronics and Advanced Materials, vol. Vol. 13, No. 5, pp. 491-496, May 2011. 
[14] C. Lerma Arce, K. De Vos, T. Claes, K. Komorowska, D. Van Thourhout and P. Bienstman, "Silicon-on-insulator microring resonator sensor integrated on an optical fiber facet," IEEE Photonics Technology Letters, vol. 23, pp. 890-892, 2011.

[15] B. Wang, T. Siahaan, M. A. Dundar, R. Notzel, D. H. Van, S. He and D. H. Van, "Photonic crystal cavity on optical fiber facet for refractive index sensing," Opt. Lett., vol. 37, pp. 833-835, 2012.

[16] G. Shambat, J. Provine, K. Rivoire, T. Sarmiento, J. Harris and J. Vuckovic, "Optical fiber tips functionalized with semiconductor photonic crystal cavities," Appl. Phys. Lett., vol. 99, 2011.

[17] K. Okamoto, Fundamentals of Optical Waveguides, Second Edition. Academic Press, 2005.

[18] C. Raum, "Thermo-mechanically tunable Bragg grating filters on silicon-oninsulator rib waveguide bridges," 2010.

[19] J. Colladon, "On the reflections of a ray of light inside a parabolic liquid stream," Comptes Rendus, vol. 15, pp. 800-802, 1842.

[20] W. H. Bragg, "X-rays and crystals," Nature, vol. 90, pp. 219, 10/24, 1912.

[21] W. L. Bragg, "Diffraction of short electromagnetic waves by a crystal," Proceedings of the Cambridge Philosophical Society, vol. 17, pp. 43-57, 02/14, 1913.

[22] Nobelprize.org. The nobel prize in physics 1915. 2012(5/11), Available: http:/www.nobelprize.org/nobel prizes/physics/laureates/1915/.

[23] A. Yariv and R. C. C. Leite, "Dielectric-waveguide mode of light propagation in p-n junctions," Appl. Phys. Lett., vol. 2, pp. 55-57, 02/01, 1963.

[24] R. Shubert and J. H. Harris, "Optical surface waves on thin films and their application to integrated data processors," IEEE Trans. Microwave Theory Tech., vol. MTT-16, pp. 1048-1054, 1968.

[25] C. Chen, Foundations for Guided-Wave Optics. Wiley-Interscience, 2006.

[26] C. Chen, "Development and Implementation of Novel Numerical Techniques for Integrated Optics and Microwave Planar Structures," 2000.

[27] Y. El Hassouani, C. Li, Y. Pennec, E. H. El Boudouti, H. Larabi, A. Akjouj, O. Bou Matar, V. Laude, N. Papanikolaou, A. Martinez and B. D. Rouhani, "Dual phononic and photonic band gaps in a periodic array of pillars deposited on a thin plate," Physical Review B (Condensed Matter and Materials Physics), vol. 82, pp. 155405 (7 pp.), 10/15, 2010.

[28] R. E. Collin, Field Theory of Guided Waves. New York : IEEE ; c 1991., 1991.

[29] A. Yariv and P. Yeh, Optical Waves in Crystals : Propagation and Control of Laser Radiation (Wiley Series in Pure and Applied Optics). Wiley-Interscience, 2002. 
[30] A. F. Oskooi, D. Roundy, M. Ibanescu, P. Bermel, J. D. Joannopoulos and S. G. Johnson, "Meep: A flexible free-software package for electromagnetic simulations by the FDTD method," Comput. Phys. Commun., vol. 181, pp. 687-702, 2010.

[31] I. Giuntoni, M. Krause, H. Renner, J. Bruns, A. Gajda, E. Brinkmeyer and K. Petermann, "Numerical survey on bragg reflectors in silicon-on-insulator waveguides," in 2008 5th International Conference on Group IV Photonics, GFP, September 17, 2008 September 19, 2008, pp. 285-287.

[32] L. Poladian, F. Ladouceur and P. D. Miller, "Effects of surface roughness on gratings," Journal of the Optical Society of America B (Optical Physics), vol. 14, pp. 1339-44, 06, 1997.

[33] P. Y. Kuan, A. Delage, J. Lapointe, B. Lamontagne, J. H. Schmid, P. Waldron, B. A. Syrett and S. Janz, "Correlation of scattering loss, sidewall roughness and waveguide width in silicon-on-insulator (SOI) ridge waveguides," J. Lightwave Technol., vol. 27, pp. 3999-4008, 2009.

[34] S. Fujii, S. Kuroki, M. Numata, K. Kotani and T. Ito, "Roughness reduction in polycrystalline silicon thin films formed by continuous-wave laser lateral crystallization with cap $\mathrm{SiO} 2$ thin films," Japanese Journal of Applied Physics, vol. 48, pp. 04-129, 04, 2009.

[35] W. Huang, X. Li, C. Xu, X. Hong, C. Xu and W. Liang, "Optical transceivers for fiber-to-the-premises applications: System requirements and enabling technologies," in 2007, pp. 11-27.

[36] A. W. Fang, G. Fish and E. Hall, "Heterogeneous photonic integrated circuits," in Optoelectronic Interconnects XII, January 23, 2012 - January 25, 2012, pp. The Society of Photo-Optical Instrumentation Engineers (SPIE).

[37] A. Weirich, "Monolithic photonic integrated circuits for $10 \mathrm{G}$ passive optical networks," Photonics Spectra, vol. 44, 2010.

[38] Anonymous "New products," Communications Magazine, IEEE, vol. 48, pp. 42-42, 2010.

[39] D. Feng, B. J. Luff and M. Asghari, "Recent advances in manufactured silicon photonics," in Optoelectronic Integrated Circuits XIV. January 25, 2012 - January 26, 2012, pp. The Society of Photo-Optical Instrumentation Engineers (SPIE).

[40] S. H. Law and L. Poladian, "Effect of waveguide optical parameters on alignment tolerances for fibre attachment," Electronic Components and Technology Conference, 2002. Proceedings. 52nd, pp. 1694-1698, 2002.

[41] L. Wosinski, Z. Wang, F. Lou, D. Dai, S. Lourdudoss and L. Thylen, "Advanced silicon device technologies for optical interconnects," in Optoelectronic Integrated Circuits XIV, January 25, 2012 - January 26, 2012, pp. The Society of Photo-Optical Instrumentation Engineers (SPIE). 
[42] R. M. Briggs, J. Grandidier, S. P. Burgos, E. Feigenbaum and H. A. Atwater, "Efficient coupling between dielectric-loaded plasmonic and silicon photonic waveguides," Nano Letters, vol. 10, pp. 4851-4857, 2010.

[43] P. Prabhathan, V. M. Murukeshan and J. Zhang, "Compact SOI nanowire refractive index sensor using phase shifted Bragg grating," Optics Express, vol. 17, pp. 1533015341, 2009.

[44] S. Mandal, R. Akhmechet, L. Chen, S. Nugen, A. Baeumner and D. Erickson, "Nanoscale optofluidic sensor arrays for dengue virus detection," in Nanoengineering: Fabrication, Properties, Optics, and Devices IV, August 27, 2007 - August 30, 2007, pp. The International Society for Optical Engineering (SPIE).

[45] V. M. N. Passaro, R. Loiacono, G. D'Amico and F. De Leonardis, "Design of Bragg grating sensors based on submicrometer optical rib waveguides in SOI," IEEE Sensors Journal, vol. 8, pp. 1603-11, 2008.

[46] P. Debackere, S. Scheerlinck, P. Bienstman and R. Baets, "Surface plasmon interferometer in silicon-on-insulator: novel concept for anintegrated biosensor," Opt. Express, vol. 14, pp. 7063-7072, 08/07, 2006.

[47] Anonymous "Rewiring the home," Nat. Photon., vol. 1, pp. 131, 2007.

[48] D. Jin, H. Chen, A. Barklund, J. Mallari, G. Yu, E. Miller and R. Dinu, "EO polymer modulators reliability study," in Organic Photonic Materials and Devices XII, San Francisco, CA, 2010.

[49] C. T. DeRose, R. Himmelhuber, D. Mathine, R. A. Norwood, J. Luo, A. K. Jen and N. Peyghambarian, "High n strip-loaded electro-optic polymer waveguide modulator with low insertion loss," Optics Express, vol. 17, pp. 3316-3321, 2009.

[50] Enami, Y., Derose, C. T., Mathine, D., Loychik, C., Greenlee, C., Norwood, R. A., Kim, T. D., Luo, J., Tian, Y., Jen, A. K. Y. and Peyghambarian, N., "Hybrid polymer//sol-gel waveguide modulators with exceptionally large electro-optic coefficients," Nat Photon, vol. 1, pp. 180-185, print, 2007.

[51] I. E. Araci, R. Himmelhuber, C. T. DeRose, J. D. Luo, A. K. -. Jen, R. A. Norwood and N. Peyghambarian, "Alignment-free fabrication of a hybrid electro-optic polymer/ion-exchange glass coplanar modulator," Optics Express, vol. 18, pp. 2103821046, 2010.

[52] Y. Sakurai and F. Koyama, "Proposal of tunable hollow waveguide distributed bragg reflectors," Jpn J Appl Phys Part 2 Letter, vol. 43, pp. L631-L633, 2004.

[53] J. Albert and Gar Lam Yip, "Insertion loss reduction between single-mode fibers and diffused channel waveguides," Appl. Opt., vol. 27, pp. 4837-43, 12/01, 1988.

[54] D. Celo, E. Post, M. Summers, T. Smy, M. J. Brett and J. Albert, "Interferometric sensing platform with dielectric nanostructured thin films," Opt. Express, vol. 17, pp. 6655-6664, 04/13, 2009. 
[55] Y. Okamura, S. Yoshinaka and S. Yamamoto, "Measuring mode propagation losses of integrated optical waveguides: a simple method," Appl. Opt., vol. 22, pp. 3892-4, 12/01, 1983.

[56] S. Pissadakis, M. N. Zervas, L. Reekie and J. S. Wilkinson, "High-reflectivity bragg gratings fabricated by 248-nm excimer laser holographic ablation in thin Ta2O5 films overlaid on glass waveguides," in Laser Ablation, 2004, pp. 1093-1096.

[57] M. Hammer and O. V. Ivanova, "Effective index approximations of photonic crystal slabs: A 2-to-1-D assessment," Opt. Quant. Electron., vol. 41, pp. 267-283, 2009.

[58] A. Yariv, "Coupled-Mode Theory for Guided-Wave Optics," IEEE J. Quant. Electron., vol. QE-9, pp. 919-933, 1973.

[59] S. O. Kasap, Optoelectronics and Photonics: Principles and Practices. Prentice Hall, 2001.

[60] R. C. Gauthier, K. E. Medri and S. R. Newman, "Modal analysis and device considerations of thin high index dielectric overlay slab waveguides," Appl. Opt., 2011.

[61] R. C. Gauthier and K. E. Medri, "Glass-based silicon-clad optical device designs," Proc. of SPIE, vol. 8266, pp. 10, 2012.

[62] R. C. Gauthier, "ELEC5702 Course Notes," Winter 2007, .

[63] R. C. Alferness, "Waveguide electrooptic modulators," IEEE Trans. Microwave Theory Tech., vol. MTT-30, pp. 1121-37, 08, 1982.

[64] R. Syms and J. R. Cozens, Optical Guided Waves and Devices. London[etc.] McGraw-Hill 1992, 1992.

[65] B. E. A. Saleh and M. C. Teich, Fundamentals of Photonics. Wiley-Interscience, 1991.

[66] A. Yariv and M. Nakamura, "PERIODIC STRUCTURES FOR INTEGRATED OPTICS," IEEE J. Quant. Electron., vol. QE-13, pp. 233-253, 1977.

[67] D. C. Flanders, R. V. Schmidt, H. Kogelnik and C. V. Shank, "Grating filters for thin-film optical waveguides," Appl. Phys. Lett., vol. 24, pp. 194-6, 02/15, 1974.

[68] D. M. Sullivan, Electromagnetic Simulation using the FDTD Method Wiley-IEEE Press $<$ SKU $>$ 705-067895-F005L-0780347471, 2000.

[69] A. Taflove and S. C. Hagness, Computational Electrodynamics: The FiniteDifference Time-Domain Method, Third Edition. Artech House Publishers, 2005.

[70] A. Jaun, "Numerical Methods for Partial Differential Equations: an Overview and Applications," KTH 2D5246, Royal Institute of Technology, Stockholm, 2005.

[71] K. Yee, "Numerical solution of inital boundary value problems involving maxwell's equations in isotropic media," Antennas and Propagation, IEEE Transactions on [Legacy, Pre - 1988], vol. 14, pp. 302-307, 1966.

[72] Y. Liu, S. B. Lee, T. S. Park, K. M. Jo and S. S. Choi, "Analysis of long-period and short-period fiber bragg gratings by phase matching condition," in Fiber Optic 128 
Components and Optical Communication, November 4, 1996 - November 5, 1996, pp. 441-448.

[73] R. Kashyap, Fiber Bragg gratings, 458, San Diego, CA: Academic Press, 1999.

[74] J. Hong, W. Huang and T. Makino, "On the transfer matrix method for distributedfeedback waveguide devices," Lightwave Technology, Joumal of, vol. 10, pp. 18601868, 1992.

[75] Ye, Zhuo, "The theoretical study of passive and active optical devices via planewave based transfer (scattering) matrix method and other approaches" (2011). Graduate Theses and Dissertations. Iowa State University Paper 12070.

[76] O. Khayam, C. Cambournac, H. Benisty, M. Ayre, R. Brenot, G. -. Duan and W. Pernice, "In-plane Littrow lasing of broad photonic crystal waveguides," Appl. Phys. Lett., vol. 91, pp. 041111, 23 July 2007, 2007.

[77] A. H. Harvey, J. S. Gallagher and J. M. H. L. Sengers, "Revised Formulation for the Refractive Index of Water and Steam as a Function of Wavelength, Temperature and Density," Journal of Physical and Chemical Reference Data, vol. 27, pp. 761-74, 07, 1998.

[78] F. J. Bates and et al, "Polarimetry, Saccharimetry and the Sugars," National Bureau of Standards C440, U.S. Government Printing Office, 1942.

[79] R. C. Weast, Handbook of Chemistry and Physics. Cleveland, Ohio: CRC Press, 1977.

[80] A. Iadicicco, A. Cusano, A. Cutolo, R. Bernini and M. Giordano, "Thinned fiber Bragg gratings as high sensitivity refractive index sensor," IEEE Photonics Technology Letters, vol. 16, pp. 1149-1151, 2004.

[81] L. J. Sherry, S. Chang, G. C. Schatz, R. P. Van Duyne, B. J. Wiley and Y. Xia, "Localized surface plasmon resonance spectroscopy of single silver nanocubes," Nano Letters, vol. 5, pp. 2034-2038, 2005.

[82] V. G. Kravets, F. Schedin, A. V. Kabashin and A. N. Grigorenko, "Sensitivity of collective plasmon modes of gold nanoresonators to local environment," Opt. Lett., vol. 35 , pp. 956-958, 04/01, 2010.

[83] J. Albert, L. Shao and C. Caucheteur, "Tilted fiber Bragg gratings sensors," Laser \& Photonics Reviews, Wiley, 2012.

[84] I. M. White and X. Fan, "On the performance quantification of resonant refractive index sensors," Opt. Express, vol. 16, pp. 1020-1028, 01/21, 2008.

[85] H. Loock and P. D. Wentzell, "Detection limits of chemical sensors: Applications and misapplications," Sensors Actuators B: Chem., vol. 173, pp. 157-163, 10, 2012.

[86] A. Iadicicco, S. Campopiano, A. Cutolo, M. Giordano and A. Cusano, "Refractive index sensor based on microstructured fiber Bragg grating," IEEE Photonics Technology Letters, vol. 17, pp. 1250-1252, 2005. 
[87] Y. Liu, P. Hering and M. O. Scully, "An integrated optical sensor for measuring glucose concentration," Applied Physics B (Photophysics and Laser Chemistry), vol. B54, pp. 18-23, 01, 1992.

[88] G. E. Moore, "Cramming more components onto integrated circuits," Electronics, vol. 38, pp. 114, April 19, 1965.

[89] P. I. Borel, L. H. Frandsen, A. Harpoth, M. Kristensen, T. Nemi, P. Xing, J. S. Jensen and O. Sigmund, "Design and fabrication of SOI-based photonic crystal components," in Proceedings of 2004 6th International Conference on Transparent Optical Networks, 2004, pp. 271-275.

[90] I. Foster, Designing and Building Parallel Programs: Concepts and Tools for Parallel Software Engineering. Boston, MA, USA: Addison-Wesley Longman Publishing Co., Inc, 1995.

[91] G. Sharma and J. Martin, "MATLAB\&reg: A language for parallel computing," International Journal of Parallel Programming, vol. 37, pp. 3-36, 2009.

[92] G. M. Amdahl, "Validity of the single processor approach to achieving large scale computing capabilities," in Proceedings of the April 18-20, 1967, Spring Joint Computer Conference, Atlantic City, New Jersey, 1967, pp. 483-485.

[93] X. Sun and Y. Chen, "Reevaluating Amdahl's law in the multicore era," J. Parallel Distrib. Comput., vol. 70, pp. 183-188, 2010.

[94] G. Liu, H. L. Schmider and K. E. Edgecombe, "The HPCVL working template: A tool for high-performance programming," in 19th International Symposium on High Performance Computing Systems and Applications, HPCS 2005, May 15, 2005 - May 18, 2005, pp. 110-116.

[95] G. Klimeck, M. Mclennan, S. B. Brophy, I. Adams G. and M. S. Lundstrom, "NanoHUB.org: Advancing education and research in nanotechnology," Computing in Science and Engineering, vol. 10, pp. 17-23, 2008.

[96] J. Racine, "The Cygwin tools: a GNU toolkit for windows," J. Appl. Econometrics, vol. 15, pp. 331-41, 05, 2000.

[97] W. Gropp, E. Lusk and T. L. Sterling, Beowulf Cluster Computing with Linux. Cambridge, Mass.: MIT Press, 2003.

[98] Compute Canada, "Compute Servers," High Performance Computing resources, Available: https://computecanada.ca/index.php/en/resources/compute-resources-fromccdb-data, 2012. 